National Library

of Canada

Canadian Theses Service

Onawa Canada

KIA ON4
Bibliothèque nationale du Canada

Service des thèses canadiennes

NOTICE

The quality of this microform is heavily dependent upon the quality of the original thesis submitted for microfilming. Every effort has been made to ensure the highest quality of reproduction possible.

If pages are missing, contact the university which granted the degree.

Some pages may have indistinct print especially if the original pages were typed with a poor typewriter ribbon or if the university sent us an inferior photocopy.

Reproduction in full or in part of this microform is governed by the Canadian Copyright Act, R.S.C. 1970, C. C-30, and subsequent amendments.
AVIS

La qualité de cette microforme dépend grandement de la qualité de la thèse soumise au microfilmage. Nous avons tout fait pour assurer une qualité supérieure de reproduction.

S'il manque des pages, veuillez communiquer avec Puniversité qui a contéré le grade.

La qualité d'impression de certaines pages peut laisser à désirer, surtout si les pages originales ont été dactylogra. phiées a l'aide d'un ruban usé ou si l'université nous a fait parvenir une photocopie de qualité inférieure.

La reproduction, méme partielle, de cette microforme est soumise à la Loi canadienne sur le droit d'auteur, SRC 1970 , c. C-30, et ses amendements subséquents. 


\title{
EVOLUTION OF A THERMAL MIXING LAYER IN UNIFORMLY SHEARED TURBULENT FLOW
}

by

\author{
Mohsen Ferchichi
}

A thesis submitted to the School of Graduate Studies in partial fulfillment of the requirement for the degree of Master of Applied Science in

Mechanical Engineering

\section{DEPARTMENT OF MECHANICAL ENGINEERING UNIVERSITY OF OTTAWA}

(C) Mohsen Ferchichi, Ottawa, Canada, 1991 
Canadian Theses Service Service des thèses canadiennes

Onawa, Canada

The author has granted an irrevocable nonexclusive licence allowing the National Library of Canada to reproduce, loan, distribute or sell copies of his/her thesis by any means and in any form or format, making this thesis available to interested persons.

The author retains ownership of the copyright in his/her thesis. Neither the thesis nor substantial extracts from it may be printed or otherwise reproduced without his/her permission.
L'auteur a accordé une licence irrévocable et non exclusive permettant à la Bibliothèque nationale du Canada de reproduire, preter. distribuer ou vendre des copies de sa thèse de quelque manière et sous quelque forme que ce soit pour mettre des exemplaires de cette thèse à la disposition des personnes intéressées.

L'auteur conserve la propriètè du droit d'auteur qui protège sa thèse. Ni la thèse ni des extraits substantiels de celle-ci ne doivent être imprimés ou autrement reproduits sans son autorisation. 


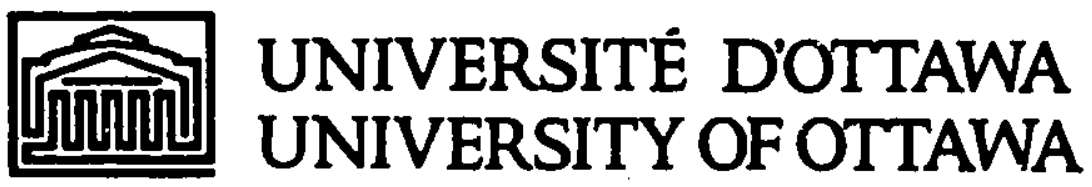




\section{Abstract}

Turbulent mixing and transport are very important phenomena due to their relevance in diverse applications (e.g. turbulent mixers, contamination of oceans and the atmosphere). For a better understanding of these phenomena, an experiment dealing with the development of a thermal mixing layer in uniformly sheared turbulent flow has been conducted. This experiment consists of introducing a stepwise increase in the mean temperature to the flow by electrically heating a set of thin ribbons, spanning half of a wind-tunnel cross-section. Hot wire anemometery and cold wire thermometry were used to measure the means and fluctuations of velocity and temperature. The downstream growth of the thermal mixirig layer and the distributions of r.m.s. temperature fluctuations, thermal fluxes and other scalar field statistics are reported. Measurements show that the thermal field reaches a quasi-asymptotic state and that the thermal mixing layer penetrated deeper into the low speed side than into the high speed side of the flow. The study also includes theoretical attempts to predict the layer's growth. 


\section{Acknowledgements}

I express deep gratitude to Professor Stavros Tavoularis for his motivating guidance and encouragements, for providing a comfortable working atmosphere and for being always available for discussions. His comments concerning experimental procedures and theoretical study contributed significantly to the quality of the thesis. I wish to thank Dr. U. Karnik for his continuous help with the instumentation and data aquisition and from whom, I learned a lot, as well as Sadok Guellouz for continuous discussions concerning "turbulent matters", also my colleagues and friends with whom I shared a warm academic experience.

I extend my thanks to Don Seaman, the department's administrative officer, and the technical staff of the departmental workshop.

The financial support provided by the Scientific Mission of Tunisia and Natural Sciences and Engineering Research Council of Canada (NSERC), is greatly appreciated.

Finally, I am indebted to my mother, Habiba, and my father, Salah, for their consistent moral support. 


\section{Contents}

Abstract

Acknowledgement

Table of contents

List of Figures iv

List of Tables v v

Nomenclature vi vi

1 Introduction 1

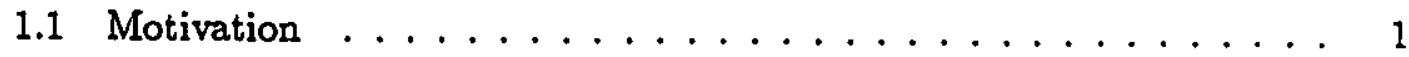

1.2 Literature Review ...................... 2 
1.2.1 General Comments .................. 2

1.2.2 Thermal Mixing Layers in Isotropic Turbulence. . . . . . . 3

1.2.3 Diffusion of Heat in Uniformly Sheared Flow. . . . . . . . 4

1.3 Objectives of the Present Research . . . . . . . . . . 6

2 Mathematical Description of the Flow 7

2.1 Scalar Transport Equations .................. $\ldots$ T

2.1.1 Balance Equation for the Instantaneous Temperature . . . $t$

2.1.2 Balance Equation for the Mean Temperature ........ S

2.i.3 Balance Equation for the Mean Squared Temperature Fluc-

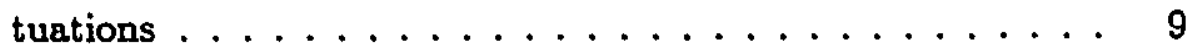

2.1.4 Balance Equation for the Turbulent Heat Flux ....... 10

2.2 Turbulent Kinetic Energy in Uniformly Sheared Turbulence . . . . 10

3 Definitions and Background $\quad 12$

3.1 Moments and Correlations . . . . . . . . . . . . . 12

3.2 Integral Length Scales . . . . . . . . . . . . . . . 13

3.3 Taylor and Corrsin Microscales ... . . . . . . . . . . . 14

4 Theoretical Predictions of the Growth of the Mixing Layer 16 
4.1 A Simplified Equation for the Mean Temperature . . . . . . . . 16

4.2 An Approximate Self-Similar Solution . . . . . . . . . . . i i

4.3 Direct Solutions ...................... 19

5 Experimental Apparatus and Measurement Procedure 22

5.1 The Flow Facility ................... 22

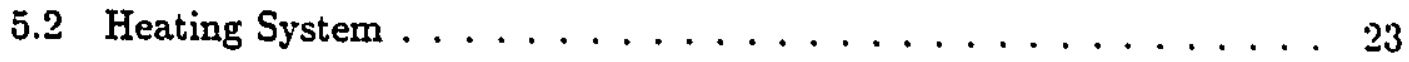

5.3 Calibration Jet $\ldots \ldots \ldots \ldots \ldots \ldots$

5.4 Velocity Measurements ................... 24

5.4.1 The Hot Wire Anemometry ............... 24

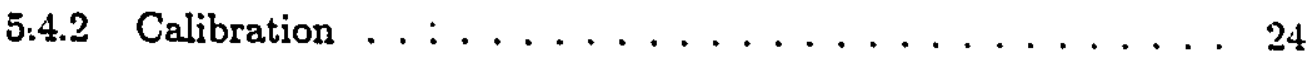

5.5 Temperature Measurements . . . . . . . . . . . . . . 26

5.5 .1 Mean Temperature ............... 26

5.5 .2 Temperature Fluctuations . . . . . . . . . . . 27

5.6 Signal Conditioning and Data Acquisition . . . . . . . . . 28

6 Measurements $\quad 30$

6.1 Velocity Measurements .................. 30

6.1 Free Stream Measurements . . . . . . . . . . . 30 
6.1.2 The Mean Velocity Field . . . . . . . . . . . . 30

6.1.3 Reynolds Stresses.................... 31

6.1.4 Integral Length Scales and Microscales. . . . . . . . . . 32

6.2 Temperature Measurements ................... 32

6.2 .1 Initial Temperature Field ................. 32

6.2.2 Mean Temperature ................. 33

6.2.3 Temperature Fluctuations . . . . . . . . . . . . 33

6.2.4 Temperature-Velocity Correlations. . . . . . . . . . . . . 34

7 Analysis and Discussion of Results

7.1 Similarity of the Mean Temperature Profiles . . . . . . . . . 35

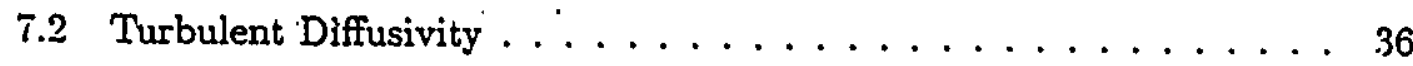

7.3 Comparison with the Approximate Solution . . . . . . . . 36

7.4 Comparison with Isotropic Diffusion $\ldots \ldots \ldots \ldots$. . . . . . $\ldots$ S

7.5 Temperature Fluctuations . . . . . . . . . . . . . 39

7.6 Temperature Velocity Covariances . . . . . . . . . . . . 40

8 Conclusion

Bibliography 
A Tables Containing Measured and Estimated Quantities 


\section{List of Figures}

5.1 Upstream section of the wind tunnel ............. 47

5.2 Shear generator. . . . . . . . . . . . . . . 48

5.3 The heating system. ....................... 49

5.4 Sketch of the wind tunnel test section. . . . . . . . . . . 50

5.5 The calibration jet. ..................... 51

5.6 Typical calibration curve for the pressure transducer. . . . . . . . 52

5.7 Typical calibration curve for hot wires ............. 53

5.8 Thermistor circuit diagram. . . . . . . . . . . . . 54

5.9 Typical calibration curves for thermistors. . . . . . . . . 55

5.10 Constant current circuit. . . . . . . . . . . . . . 56

5.11 Typical calibration curve for cold wire. . . . . . . . . . . . 57

6.1 Free stream velocity measurements. . . . . . . . . . . 58

6.2 Turbulent intensity in the empty tunnel. . . . . . . . . . . 59 
6.3 Mean velocity profiles. . . . . . . . . . . . . 60

6.4 Variations of the streamwise and transverse turbulent intensity. . . . 61

6.5 Shear stress coefficient. . . . . . . . . . . . . . 62

6.6 Streamwise growth of the turbulent stresses. . . . . . . . . 63

6.7 Integral length scales growth. . . . . . . . . . . . . 64

$6 . S$ Streamwise growth of $L_{11} \ldots \ldots \ldots \ldots$

6.9 Transverse profiles of Taylor microscales. . . . . . . . . . . . 66

6.10 Streamwise profiles of Taylor microscales. . . . . . . . . . . 67

6.11 Velocity profiles with the presence of the heating ribbons. . . . . $6 S$

6.12 Transverse profiles of the mean temperature rise. . . . . . . . 69

6.13 Isotherms on the wind tunnel centerplane. . . . . . . . . . 72

6.14 Growth of the mixing layer's width. . . . . . . . . . . 73

6.15 Transverse profiles of the temperature variance. . . . . . . . . T4

6.16 Locus of the temperature variance peaks. . . . . . . . . . 76

6.17 Transverse variation of the temperature fluctuation skewness factor. $\quad 77$

6.18 Transverse variation of the streamwise heat flux coefficient. . . . . 78

6.19 Transverse variation of the transverse heat flux coefficient. . . . . . . 80

6.20 Centerline streamwise heat flux coefficient. . . . . . . . . . . 82 
6.21 Centerline transverse heat flux coefficient. . . . . . . . 83

6.22 Triple correlation coefficient $\ldots \ldots \ldots \ldots \ldots \ldots \ldots \ldots$

6.23 Triple correlation coefficient $\ldots \ldots \ldots \ldots \ldots \ldots \ldots \ldots$

7.1 Dimensionless maximum temperature gradient. . . . . . . 86

7.2 Dimensionless maximum temperature gradient. . . . . . . s7

7.3 Collapse of all mean temperature profiles. . . . . . . . s 8

7.4 Estimate of the turbulent diffusivity, $D_{22} \ldots \ldots \ldots \ldots$. . . 89

7.5 Turbulent diffusivity normalized with Eulerian scales. . . . . . 90

7.6 Comparison of width with the approximate solution. . . . . . 91

7.7 Comparison of the mean temperature field with the approximate solution. . . . . . . . . . . . . . . . . 92

7.8 Comparison of thermal mixing layer width with the isotropic case. . 93

7.9 Ratio of temperature to velocity streamwise length scales. . . . . 94

7.10 Normalized peak r.m.s. temperature fluctuations. . . . . . . . 95 


\section{List of Tables}

A.1 Measurements at $x_{1} / M=40 \ldots \ldots \ldots \ldots$. . . . . .

A.2 Measurements at $x_{1} / M=50 \ldots \ldots \ldots \ldots$. . . . . .

A.3 Measurements at $x_{1} / M=60 \ldots \ldots \ldots \ldots \ldots$

A.4 Measurements at $x_{1} / M=80 \ldots \ldots \ldots \ldots$

A.5 Measurements at $x_{1} / M=90 \ldots \ldots \ldots \ldots 1$

A.6 Measurements at $x_{1} / M=105 \ldots \ldots \ldots \ldots \ldots \ldots$. . . . . . . . . .

A.7 Measured and estimated centerline quantities. . . . . . . . . . 103 


\title{
Nomenclature
}

\author{
Do coefficient of turbulent diffusivity \\ $D_{i j} \quad$ turbulent diffusivity tensor \\ $E \quad$ voltage \\ $h$ wind tunnel height \\ $J$ Bessel function \\ $k$ exponent in kinetic energy growth law \\ $k_{D}$ exponent in turbulent diffusivity equation \\ $\ell \quad$ characteristic length scale \\ $L_{i j, k} \quad$ velocity integral length scale \\ $L_{\theta} \quad$ temperature integral length scale \\ $M \quad$ mesh size, screen spacing \\ $P \quad$ pressure \\ $q \quad$ turbulent kinetic energy
}


$q_{r} \quad$ reference turbulent kinetic energy

$R_{x} \quad$ autocorrelation

$S_{\theta} \quad$ temperature fluctuations skewness factor

$t$ time

$\bar{T}$ mean temperature

$T_{11} \quad$ time scale

$\bar{T}_{m} \quad$ maximum mean temperature

$T_{a} \quad$ fluid temperature

$T_{w} \quad$ wire temperature

$u_{i} \quad$ velocity fluctuations ( $\left.\mathrm{i}=1,2,3\right)$

$u_{i}^{\prime} \quad$ r.m.s. value of velocity fluctuations $(i=1,2,3)$

$\bar{U}_{1} \quad$ streamwise velocity

$\bar{U}_{c} \quad$ centerline velocity

$\bar{U}_{\text {eff }}$ hypothetical velocity in King's law

$x_{i} \quad$ catesian coordinates $(\mathrm{i}=1,2,3)$

$x_{r} \quad$ reference centerline distance 


\title{
Greek letters
}

\author{
$\beta$ separation variable \\ $\gamma$ molecular diffusivity \\ $\epsilon \quad$ dissipation of kinetic energy \\ $\epsilon_{\theta} \quad$ dissipation of temperature \\ $\lambda_{i i} \quad$ Taylor microscale \\ $\lambda_{\theta} \quad$ Corrsin microscale \\ $\nu \quad$ kinematic viscosity \\ $\omega \quad$ width of the mixing layer \\ $\rho_{u_{1} u_{2}} \quad$ turbulent shear stress coefficient \\ $\rho_{u_{1} \theta} \quad$ turbulent shear stress coefficient \\ $\rho_{\mathrm{u}_{2} \theta} \quad$ turbulent shear stress coefficient \\ $\theta$ temperature fluctuation \\ $\theta^{\prime} \quad$ r.m.s. value of temperature fluctuations
}




\section{Chapter 1}

\section{Introduction}

\subsection{Motivation}

Diffusion and mixing of scalar quantities by a turbulent flow are topics of great interest, due to their relevance in diverse applications such as the contamination of oceans, inland waters and the atmosphere, the control of chemical reactions in turbulent mixers and the mixing of air and carbon dioxide above plants and crop canopies. In these applications, diffusion and mixing are due primarily to turbulent motions, while the molecular action is responsible for reducing the inhomogeneity of scalar concentration at a scale that is much smaller than the size of turbulent eddies.

Although most flows of technological interest are quite complex, fundamental research on turbulent diffusion has focussed upon a few, simplified flow configurations, which, presumably, display some of the important features of more complex flow. One case that has been studied extensively is that of isotropic turbulence (i.e a flow whose statistical properties are independent of direction ). At the same time, 
it has been established that turbulent flows in the atmosphere and the oceans or in various types of machinery are not isotropic and that shearing due to velocity gradient has an important effect on the process of mixing of contaminants in such flows. Therefore, the study of diffusion of scalars in a simplified laboratory flow, which is subject to shear, would be useful in elucidating the mechanisms of diffusion in environmental and technological flows. In particular, the effect of uniform shear in the mixing of two streams with different mean scalar concentrations has not yet been investigated analytically or experimentally. This will be the subject of the present study.

\subsection{Literature Review}

\subsubsection{General Comments}

The first detailed study of turbulent diffusion was the pioneering work of Taylor (1921). Since then, many others have conducted further theoretical as well as experimental research regarding the scalar fluctuation field in different flow configurations. A summary of earlier work can be found in the book by Monin and Yaglom (1971).

In most experimental studies of turbulent diffusion and mixing, temperature difference was chosen as the transported scalar because it can be generated and measured relatively easily. As long as the temperature differences are small (typically no more than few degrees Kelvin), then the temperature can be considered as "passive" scalar, namely having negligible effect on the velocity statistics. Heat dif- 
fusion in wind tunnel flows has been studied by generating temperature differences using distributed heat sources, such as electrically heated grids, screens and other devices or concentrated sources such as heated wires and ribbons. One concern of several researchers was to avoid any significant distortion of the velocity field by the physical presence of the heaters or the heat itself. The present literature review will focus on two topics, which appear to be most relevant to the present work : studies of thermal mixing layer in grid-generated, nearly isotropic turbulence and studies of heat diffusion in uniformly sheared flows.

\subsubsection{Thermal Mixing Layers in Isotropic Turbulence.}

A number of experimental studies of thermal mixing layer developing downstream of partially heated grid or screen have been already published. The pioneering experiment related to this flow is apparently due to Watt and Baines (1973), followed by the work of Libby (1975) and Keffer et al. (1977).

Attempts to provide similarity solutions for this type of flows were reported by Libby (1975), LaRue and Libby (1981) and LaRue et al. (1281). All these authors have concluded that the mean temperature field would approach an error function-like distribution, whereas, the temperature fluctuations would approach a Gaussian distribution and that both functions would become independent of downstream position when plotted against the similarity variable $\eta=y x^{-1 / 2},(x$ and $y$ are the streamwise and transverse coordinates). However, experimental results by the same authors showed a peak of the r.m.s. temperature fluctuations that was 
$40 \%$ smaller than the predicted value. To remove this discrepancy, LaRue et al. (1981) select:d a virtual origin that was different from the one applying to the rate of the decay of temperature fluctuations in the fully heated stream and obtained a good agreement between the predicted and measured r.m.s. temperature fluctuations.

Ma and Warhaft (1986) introduced heat into the flow using a half-heated screen of thin wires in order to decouple the velocity and temperature fluctuations at their origins. They concluded that the temperature fluctuation field was not self-preserving and they attributed this behaviour to the difference in the growth rates of the temperature and velocity length scales. Gibson et al. (1989) provided measurements of the thermal mixing layer far downstream of a half-heated screen at higher Reynolds number than in previously reported experiments. Their experimental results, taken at distances of up to 400 mesh sizes of the grid, show that these flows apparently do not approach a self-similar state.

\subsubsection{Diffusion of Heat in Uniformly Sheared Flow.}

Corrsin (1952) was one of the first to investigate the turbulent diffusion in homogeneous shear flows. Since then, many others conducted experimental as well as theoretical work on this topic. Theories on turbulent diffusion in shear flows have been reviewed by Hinze (1975). Turbulent diffusion in homogeneous shear flow with a temperature profile having constant mean gradient has been studied by Fox (1964), Tavoularis and Corrsin (1981 and 1985), and Sreenivasan et al. (1982). More specifically, Sreenivasan et al.(1982) evaluated the performance of gradient 
transport models, whereas Tavoularis and Corrsin (19S5) focused on the effect of the mean shear on the turbulent diffusivity tensor.

Theoretical and computational studies describing the diffusion of contaminants in homogeneous, sheared turbulent flow have been reported by Riley and Corrsin (1971,1974), Novikov (1958), Huang (1979) and Okubo and Karweit (197i). A few analyses and experiments have considered the case of diffusion of heat from a line source in uniformly sheared flow. Chung and Kyong (19S9) performed mensurements in weakly sheared turbulence and reported that the mean temperature profiles as well as the fluctuating temperature field approached Gaussian shapes; their paper also inci:ided a model for higher order scalar transport quantities. Stilpountzis and Britter (1987) made measurements relatively close to the line sourcc and also reported that the mean and fluctuating temperature approached Gaussian shapes. Karnik and Tavoularis (1989) provided a detailed report on the evolution of the mean and fluctuating temperatures. Their measurements indicated that the mean temperature profile was approximately Gaussian near the source line, but became asymmetric far downstream and its peak shifted towards the low velocity region. The temperature fluctuation profile double-peaked near the source, it had one peak at intermediate distances and demonstrated a re-emergence of double peaks which grew in relative magnitude far downstream. They also reported that the measured heat fluxes and the triple temperature-velocity correlations demonstrated self-similar features.

Nakamura et al.(1986) and Sakai et al.(1986) performed measurements related to the diffusion of matter from a point source in a uniformly sheared turbulent flow of water. Finally Rogers et al (1989) have presented a model for the turbulent flux 
of passive scalars in hornogenous turbulent shear flow.

\subsection{Objectives of the Present Research}

The previous literature review has documented that no literature is available on the growth of a thermal mixing layer in the presence of uniform mean shear. The objective of the present work is to study, both theoretically and experimentally the above mentioned subject. The objective of the experimental study is to provide measurements of the mean temperature; the moments of temperature fluctuations as well as the turbulent heat fluxes across the mixing layer. "On the theoretical side, the present thesis aims at providing predictions of the temperature field for this type of flows. It is hoped that this study will contribute to the understanding of the mechanisms of turbilent diffusion and that it will lead to further research involving turbulent transpor'c and mixing. Results of the present study should also be useful in the development and testing of theoretical and computational models of scalar transport. 


\section{Chapter 2}

\section{Mathematical Description of the Flow}

\subsection{Scalar Transport Equations}

\subsubsection{Balance Equation for the Instantaneous Temperature}

The transport of a passive scalar, $\mathrm{T}$, in turbulent flow is governed by

$$
\frac{\partial T}{\partial t}+U_{j} \frac{\partial T}{\partial x_{j}}=\gamma \frac{\partial^{2} T}{\partial x_{j} \partial x_{j}}
$$

where $\gamma$ is the molecular diffusivity. Equation (2.1) suggests that the rate of change of $\mathrm{T}$ is equal to its molecular diffusion. In the following, the scalar will be referred to as temperature, however the analysis also applies to other transported scalars. Derivation of statistical equations, as required for the description of a random field, can be made using the Reynolds decomposition, as

$$
\begin{gathered}
T=\bar{T}+\theta \\
U_{j}=\overline{U_{j}}+u_{j}
\end{gathered}
$$


where the overbared quantities designate the mean values and lower case quantities represent the fluctuations arcund the means.

Substituting equations (2.2) and (2.3) in equation (2.1) yields

$$
\frac{\partial \bar{T}}{\partial t}+\frac{\partial \theta}{\partial t}+\left(\overline{U_{j}}+u_{j}\right)\left(\frac{\partial \bar{T}}{\partial x_{j}}+\frac{\partial \theta}{\partial x_{j}}\right)=\gamma \frac{\partial^{2} \bar{T}}{\partial x_{j} \partial x_{j}}+\gamma \frac{\partial^{2} \theta}{\partial x_{j} \partial x_{j}}
$$

\subsubsection{Balance Equation for the Mean Temperature}

Averaging equation (2.4), all the fluctuating quantities vanish and the balance equation for the mean temperature is obtained as

$$
\frac{\partial \bar{T}}{\partial t}+\overline{U_{j}} \frac{\partial \bar{T}}{\partial x_{j}}=\gamma \frac{\partial^{2} \bar{T}}{\partial x_{j} \partial x_{j}}-\frac{\partial \overline{\theta u_{j}}}{\partial x_{j}}
$$

Compared to equation (2.1), equation (2.5) has an additional term (last term in equation (2.5)), which represents the turbulent convection of temperature fluctuations.

In the case of uniformly sheared turbulent flow, with the assumption that the flow is stationary and two-dimensional on the mean, the balance equation for the mean temperature becomes

$$
\overline{U_{1}} \frac{\partial \bar{T}}{\partial x_{1}}=\gamma\left(\frac{\partial^{2} \bar{T}}{\partial x_{1}^{2}}+\frac{\partial^{2} \bar{T}}{\partial x_{2}^{2}}\right)-\left(\frac{\partial \overline{u_{1} \partial}}{\partial x_{1}}+\frac{\partial \overline{u_{2} \theta}}{\partial x_{2}}\right)
$$




\subsubsection{Balance Equation for the Mean Squared Temperature Fluctuations}

The balance equation for the temperature fluctuations is obtained by subtracting equation (2.5) from equation (2.4) so as to obtain first the following

$$
\frac{\partial \theta}{\partial t}+\overline{U_{j}} \frac{\partial \theta}{\partial x_{j}}+u_{j} \frac{\partial \bar{T}}{\partial x_{j}}+\frac{\partial \theta u_{j}}{\partial x_{j}}-\frac{\partial \overline{\theta u_{j}}}{\partial x_{j}}=\gamma \frac{\partial^{2} \theta}{\partial x_{j} \partial x_{j}}
$$

Multiplying equation (2.7) by $\theta$ and averaging, one obtains an equation for the mean squared temperature fluctuations as

$$
\frac{\partial \overline{\theta^{2}}}{\partial t}+\overline{U_{j}} \frac{\partial \overline{\theta^{2}}}{\partial x_{j}}=-2 \overline{u_{j} \theta} \frac{\partial \bar{T}}{\partial x_{j}}-\frac{\partial \overline{\theta^{2} u_{j}}}{\partial x_{j} \partial x_{j}}+\gamma \frac{\partial^{2} \overline{\theta^{2}}}{\partial x_{j} \partial x_{j}}-2 \gamma \overline{\frac{\partial \theta}{\partial x_{j}} \frac{\partial \theta}{\partial x_{j}}}
$$

Equation (2.S) suggests that the total rate of change of temperature fluctuations is equal to the balance of the rate of production of $\overline{\theta^{2}}$ by the mean gradient, the rate of transport of temperature fluctuations by turbulent velocity fiuctuations, the molecular diffusion of $\overline{\theta^{2}}$ and the rate of destruction of $\overline{\theta^{2}}$ by molecular actions. In a stationary uniformly sheared turbulent flow, the above equation reduces to

$$
\bar{U}_{1} \frac{\partial \overline{\theta^{2}}}{\partial x_{1}}=-2 \overline{u_{2} \theta} \frac{\partial \bar{T}}{\partial x_{2}}-\frac{\partial \overline{\theta^{2} u_{2}}}{\partial x_{2}}+\gamma \frac{\partial^{2} \overline{\theta^{2}}}{\partial x_{2}^{2}}-2 \epsilon_{\theta}
$$

where $2 \epsilon_{\theta}$ denotes the dissipation of temperature fluctuations, equal to the last term. in equation (2.8), and streamwise gradients have been neglected compared to transverse gradients. 


\subsubsection{Balance Equation for the Turbulent Heat Flux}

The equation for the velocity-temperature covariance (or heat flux) $\overline{\theta u_{i}}$ can be obtained as follows:

$$
\frac{\partial \overline{\theta u_{i}}}{\partial t}+\overline{U_{j}} \frac{\partial \overline{\theta u_{i}}}{\partial x_{j}}=-\frac{\partial \overline{\theta u_{i} u_{j}}}{\partial x_{j}}-\overline{u_{i} u_{j}} \frac{\partial \bar{T}}{\partial x_{j}}-\overline{\theta u_{j}} \frac{\partial \overline{U_{i}}}{\partial x_{j}}-\frac{1}{\rho} \theta \overline{\frac{\partial p}{\partial x_{i}}}+\frac{\partial}{\partial x_{j}}\left(\gamma \overline{u_{i} \frac{\partial \theta}{\partial x_{j}}}+\nu \overline{\frac{\partial u_{i}}{\partial x_{j}}}\right)-(\nu+\gamma) \frac{\partial \overline{u_{i}}}{\partial x_{j}} \frac{\partial \theta}{\partial x_{j}}
$$

In the case of a stationary uniformly sheared turbulent flow, the above equation reduces to

$\overline{U_{1}} \frac{\partial \overline{\theta u_{1}}}{\partial x_{1}}=-\frac{\partial \overline{\theta u_{1} u_{2}}}{\partial x_{2}}-\overline{u_{1} u_{2}} \frac{\partial \bar{T}}{\partial x_{2}}-\overline{\theta u_{2}} \frac{\partial \overline{U_{1}}}{\partial x_{2}}-\frac{1}{\rho} \theta \overline{\frac{\partial p}{\partial x_{1}}}+\frac{\partial}{\partial x_{2}}\left(\gamma \overline{u_{1} \frac{\partial \theta}{\partial x_{2}}}+\nu \overline{\theta \frac{\partial u_{1}}{\partial x_{2}}}\right)-(\nu+\gamma) \frac{\overline{\partial u_{1}}}{\partial x_{j}} \frac{\partial \theta}{\partial x_{j}}$

and

$$
\overline{U_{1}} \frac{\partial \overline{\theta u_{2}}}{\partial x_{1}}=-\frac{\partial \overline{\theta u_{2}^{2}}}{\partial x_{2}}-\overline{u_{2}^{2}} \frac{\partial \bar{T}}{\partial x_{2}}-\frac{1}{\rho} \theta \overline{\frac{\partial p}{\partial x_{2}}}+\frac{\partial}{\partial x_{2}}\left(\gamma \overline{u_{2} \frac{\partial \theta}{\partial x_{2}}}+\nu \overline{\frac{\partial u_{1}}{\partial x_{2}}}\right)-(\nu+\gamma) \overline{\frac{\partial u_{2}}{\partial x_{j}} \frac{\partial \theta}{\partial x_{j}}}
$$

\subsection{Turbulent Kinetic Energy in Uniformly Sheared Turbulence}

In uniformly sheared turbulent flow, the simplified form of the turbulent kinetic energy equation is given by (Tavoularis, 1985) as

$$
\overline{U_{1}} \frac{d\left(\frac{1}{2} q^{2}\right)}{d x_{1}}=-\overline{u_{1} u_{2}} \frac{d \overline{U_{1}}}{d x_{2}}-\nu \overline{\frac{\partial u_{i}}{\partial x_{j}} \frac{\partial u_{i}}{\partial x_{j}}}
$$

where the turbulent kinetic energy per unit mass is

$$
\frac{1}{2} q^{2}=\frac{1}{2}\left(\overline{u^{2}}+\overline{v^{2}}+\overline{w^{2}}\right)
$$


Tavoularis (1985) also showed that the turbulent kinetic energy grows exponentially with the streamwise direction as

$$
q\left(x_{1}\right)=q_{r} e^{\frac{\left(x_{1}-x_{r}\right)}{r}}
$$

where $q_{r}, x_{r}$ are reference values, $\ell$ is a characteristic length given by

$$
\ell=\left[\frac{K_{12}}{\overline{U_{1}}} \frac{d \overline{U_{1}}}{d x_{2}}-\frac{\epsilon}{\overline{U_{1}} q^{2}}\right]^{-1}
$$

and

$$
\Pi_{12}=\frac{\overline{u_{1} u_{2}}}{q^{2}}=\text { constant }
$$




\section{Chapter 3}

\section{Definitions and Background}

Turbulent flows are highly irregular, so that quantities describing the flow are always random in space and time and require a statistical approach for their definition.

\subsection{Moments and Correlations}

The distribution function $F_{\chi}(x)$ of a random variable, $\chi$, is by definition the probability that $\chi \leq x$. The probability density function of $\chi$ is defined as the derivative of the distribution function as

$$
f_{\chi}(x)=\frac{d F_{\chi}(x)}{d x}
$$

In the case of a stationary (the statistical properties do not depend on time origin) and ergodic (time averages are equal to ensemble averages) random process, the mean can be computed as

$$
\bar{x}=\lim _{T \rightarrow \infty} \frac{1}{2 T} \int_{-T}^{T} \chi(t) d t
$$


where $T$ is a time interval.

The variance of the random variable $\chi$ is

$$
\sigma^{2}=\overline{(x-\bar{x})^{2}}=\int_{-\infty}^{\infty} \overline{(x-\bar{x})^{2}} f_{x}(x) d x
$$

The positive square root $\sigma$ is called the standard deviation or the root mean squared value of the random variable $x$.

The "skewness factor" represents the degree of symmetry of a distribution and it is defined as follows:

$$
S=\frac{\overline{(x-\bar{x})^{3}}}{\sigma^{3}}
$$

For a stationary and ergodic random process, the autocorrelation function is defined as

$$
R_{x}(\tau)=\lim _{T \rightarrow \infty} \frac{1}{2 T} \int_{-T}^{T} \chi(t+\tau) \chi(t) d t
$$

while, for two jointly stationary random processes, the cross-correlation function is defined as

$$
R_{\chi \psi}(\tau)=\lim _{T \rightarrow \infty} \frac{1}{2 T} \int_{-T}^{T} \chi(t+\tau) \psi(t) d t
$$

\subsection{Integral Length Scales}

The integral length scales represent the order of magnitude of the size of entities with relative motions contributing mostly to the turbulent kinetic energy (energy containing eddies). The streamwise Eulerian integral length scale, $L_{11,1}$, can be determined from the corresponding integral time scale, $T_{11}$ as

$$
L_{11,1}=\bar{U} T_{11}
$$


where

$$
T_{11}=\int_{0}^{\infty} \frac{\overline{u_{1}(t) \overline{u_{1}(t+\tau)}}}{\overline{u_{1}^{2}}} d \tau
$$

Integral length scales for the temperature fluctuations can be defined by analogy to the above equations.

\subsection{Taylor and Corrsin Microscales}

The Taylor microscale can be estimated according to the definition

$$
\begin{gathered}
\lambda_{i i}=\left[\frac{\overline{u_{i}^{2}}}{\left(\frac{\partial u_{i}}{\partial x_{i}}\right)^{2}}\right]^{0.5} \quad(i \text { not summed }) \\
\lambda_{i j}=\left[\frac{2 \overline{u_{i}^{2}}}{\overline{\left(\frac{\partial u_{i}}{\partial x_{j}}\right)^{2}}}\right]^{0.5} \quad(i \neq j)
\end{gathered}
$$

Taylor's approximation, expressed as .

$$
\frac{\partial(\ldots)}{\partial x_{1}} \approx-\frac{1}{\bar{U}_{1}} \frac{\partial(\ldots)}{\partial t}
$$

can be justified for low turbulent intensity typically, less than $10 \%$ (Tavoularis, 1986). Using Taylor's approximation, the streamwise microscale, $\lambda_{11}$, can be found as

$$
\lambda_{11}=\overline{U_{1}}\left[\frac{\overline{u_{1}^{2}}}{\left(\frac{\overline{\partial u_{1}}}{\partial t}\right)^{2}}\right]^{0.5}
$$

The streamwise "Corrsin" microscale for fluctuating temperature field is defined as

$$
\lambda_{\theta_{2}}=\left[\frac{2 \overline{\theta^{2}}}{\overline{\left(\frac{\partial \theta}{\partial x_{1}}\right)^{2}}}\right]^{0.5}
$$


Using Taylor's hypothesis, the streamwise microscale can be estimated from the mean square temporal derivative of temperature as

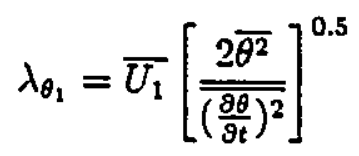




\section{Chapter 4}

\section{Theoretical Predictions of the Growth of the Mixing Layer}

\subsection{A Simplified Equation for the Mean Tem- perature}

Equation (2.6) in Chapter 2 describes the mean temperature balance for the case of uniformly sheared flow, stationary and two-dimensional on the mean. As a further simplification, the molecular diffusion of temperature can be neglected when compared to the turbulent diffusion terms. Therefore equation, (2.6) becomes

$$
\overline{U_{1}} \frac{\partial \bar{T}}{\partial x_{1}}=-\left(\frac{\partial \overline{u_{1} \theta}}{\partial x_{1}}+\frac{\partial \overline{u_{2} \theta}}{\partial x_{2}}\right)
$$

A widely used approximation in the modeling of the turbulent heat flux $\overline{\theta u_{i}}$ is based on the gradient transport assumption, which in generalized from (Tavoularis and Corrsin, 1985), can be written as:

$$
\overline{u_{i} \theta}=-D_{i j} \frac{\partial \bar{T}}{\partial x_{j}}
$$


where $D_{i j}$ is called the turbulent diffusivity tensor. Substituting equation (4.2) in equation (4.1), one gets:

$$
\bar{U}_{1} \frac{\partial \bar{T}}{\partial x_{1}}=-D_{11} \frac{\partial^{2} \bar{T}}{\partial x_{1}^{2}}+\left(D_{12}+D_{21}\right) \frac{\partial^{2} \bar{T}}{\partial x_{1} \partial x_{2}}+D_{22} \frac{\partial^{2} \bar{T}}{\partial x_{2}^{2}}
$$

The thermal mixing layer is a case where the transverse temperature gradient,

$\frac{\partial \bar{T}}{\partial x_{2}}$, is much larger than the streamwise one, $\frac{\partial \bar{T}}{\partial x_{1}}$. Then equation $(4.3)$ can be approximated by

$$
\bar{U}_{1} \frac{\partial \bar{T}}{\partial x_{1}} \approx D_{22} \frac{\partial^{2} \bar{T}}{\partial x_{2}^{2}}
$$

The turbulent diffusivity, $D_{22}$, has been customarily estimated from the product of the Eulerian scales $u_{2}^{\prime} L_{11,1}$, where $u_{2}^{\prime}$ is the velocity fluctuation in the $x_{2}$ direction and $L_{11,1}$ is the Eulerian integral. length scale defined by equation (3.14). Presumably $D_{22}$ is a function of $x_{1}$ only. Previous experiments (Tavoularis and Karnik, 1989) have established that both $u_{.2}^{\prime}$ and $L_{11,1}$ can be approximated by exponential functions of $x_{1}$, so that $D_{22}\left(x_{1}\right)$ can also be approximated by an exponential function.

\subsection{An Approximate Self-5, imilar Solution}

If, for a moment, one restricts the solution to a relatively narrow thermal mixing layer, then the mean velocity across the layer can be assumed to be constant, $\left(\bar{U}_{1}=\bar{U}_{c}\right)$. Furthermore, we assume that a similarity solution exists in the form

$$
\frac{\bar{T}\left(x_{1}, x_{2}\right)}{\overline{T_{m}}}=f(\eta)
$$

and

$$
\eta=\frac{x_{2}+x_{2,0}\left(x_{1}\right)}{g\left(x_{1}\right)}
$$


We shall make the additional assumption that the location of $x_{2,0}\left(x_{1}\right)$, of the thermal mixing layer center obeys the same law as the mixing layer scale function, $g\left(x_{1}\right)$, such that $x_{2,0}\left(x_{1}\right)=\beta g\left(x_{1}\right)$ where, $\beta$ is a constant. Substituting equations (4.5) and (4.6) in equation (4.4) and simplifying yields

$$
-\bar{U}_{c} \frac{d f}{d \eta} \frac{(\eta-\beta)}{g\left(x_{1}\right)} \frac{d g\left(x_{1}\right)}{d x_{1}}=D_{22} \frac{d^{2} f}{d \eta^{2}} \frac{1}{g^{2}\left(x_{1}\right)}
$$

This is a separable clifferential equation and can be written as

$$
-\frac{g\left(x_{1}\right)}{D_{22}} \frac{d g}{d x_{1}}=\frac{1}{\overline{U_{c}}(\eta-\beta)} \frac{1}{d f / d \eta} \frac{l^{2} f}{d \eta^{2}}=-I_{i}^{-2}
$$

which can be separated into two equations, as

$$
\frac{g\left(x_{1}\right)}{D_{22}} \frac{d g}{d x_{1}}=K^{-2}
$$

and

$$
\frac{1}{d f / d \eta} \frac{d^{2} f}{d \eta^{2}}=-U_{c \perp 1}{ }^{2}(\eta-\beta)
$$

Equation (4.10) has the solution

$$
f(\eta)=\int_{0}^{\eta} \exp \left(-\frac{\overline{U_{c}} K^{2}}{2}(\eta-\beta)^{2}\right) d \eta+\text { const }
$$

Applying the boundary conditions:

$$
\begin{aligned}
& \eta \rightarrow+\infty \Rightarrow f(\eta) \rightarrow 1 \\
& \eta \rightarrow-\infty \Rightarrow f(\eta) \rightarrow 0
\end{aligned}
$$

one gets

$$
K=\sqrt{\frac{2 \pi}{\overline{U_{c}}}}
$$


from which the mean temperature field combl be described by:

$$
\frac{\bar{T}(\eta)}{\bar{T}_{m}}=\frac{1}{2}[(r \cdot f(\sqrt{\pi}(\eta-j))+1]
$$

Equation (4.9) has the solution

$$
g\left(x_{1}\right)=\left[\frac{ \pm \pi}{\overline{\bar{t}_{2}}} \int_{0}^{x_{1}}-D_{2 \cdot 2}\left(x_{1}\right) d x_{1}+A\right]^{1 / 2}
$$

Recalling that

$$
D_{22}=D_{0} c^{k x_{1}}
$$

with $D_{0}$ and $k_{D}$ known constants for a given velocity field, one may infer that the thermal mixing layer scale function is

$$
g\left(x_{1}\right)=\left[\frac{4 \pi D_{0}}{\overline{\bar{U}_{L}} k_{D}}\left(c^{k_{D} x_{1}}-1\right)+A\right]^{\frac{1}{2}}
$$

where $A$ is to be determined from the experimental results.

The assumption of constancy of $\overline{U_{1}}$ was a crucial one for the above analysis. If, instead, one lets the velocity change linearly with $x_{2}$, one derives a differential equation which is non-separable, inclicating that sclf-similar solutions mily not cxist in this case.

\subsection{Direct Solutions}

The proposed solution in this section is based on the separation of varialsles technique. Let an elementary solution be of the form

$$
\bar{T}\left(x_{1}, x_{2}\right)=\Theta_{1}\left(x_{1}\right) \Theta_{2}\left(x_{2}\right)
$$


Then

$$
\frac{\partial \bar{T}}{\partial x_{1}}=\Theta_{2}\left(x_{2}\right) \frac{d \Theta_{2}\left(x_{1}\right)}{d x_{1}}
$$

and

$$
\frac{\partial^{2} \bar{T}}{\partial x_{2}^{2}}=\Theta_{1}\left(x_{1}\right) \frac{d^{2} \Theta_{2}\left(x_{2}\right)}{d x_{2}^{2}}
$$

Substituting equations (4.18) and (4.19) in equation (4.4), one gets

$$
\bar{U}_{1} \Theta_{2} \frac{d \Theta_{1}}{d x_{1}}=D_{22} \Theta_{1} \frac{d^{2} \Theta_{2}}{d x_{2}^{2}}
$$

or

$$
\frac{1}{\Theta_{1} D_{22}} \frac{d \Theta_{1}}{d x_{1}}=\frac{1}{\bar{U}_{1} \Theta_{2}} \frac{d^{2} \Theta_{2}}{d x_{2}^{2}}=-\beta^{2}
$$

where $-\beta^{2}$ is the separation variable, chosen to be negative, because positive value would produce growing functions that have no physical meaning. The first equation is

$$
\frac{1}{\Theta_{1}} \frac{d \Theta_{1}}{d x_{1}}=-\beta^{2} D_{22}
$$

and has the general solution

$$
\Theta_{1}\left(x_{1}\right)=A e^{\left[\int_{0}^{x_{1}}-\beta^{2} D_{22}\left(x_{1}\right) d x_{1}\right]}
$$

The second equation is

$$
\frac{1}{\bar{U}_{1} \Theta_{2}} \frac{d^{2} \Theta_{2}}{d x_{2}^{2}}=-\beta^{2}
$$

In uniformly sheared flow, the velocity is given by

$$
\bar{U}_{1}=a x_{2}+b
$$

where $\mathrm{a}, \mathrm{b}$ are known constants. Let

$$
X=a x_{2}+b
$$




$$
\Theta_{2}\left(x_{2}\right)=\Theta(X) \sqrt{X}
$$

and

$$
z=\frac{2 \beta}{3 a} X^{-\frac{2}{2}}
$$

Substituting equations (4.25), (4.26) and (4.27) in (4.24), one gets

$$
z^{2} \frac{d^{2} \Theta}{d z^{2}}+z \frac{d \Theta}{d z}++\left(z^{2}-\left(\frac{1}{3}\right)^{2}\right) \Theta=0
$$

Equation (4.28) is a Bessel equation of order $\frac{1}{3}$. Therefore, the solution for equation (4.24) is

$$
\Theta_{2}\left(x_{2}\right)=B \sqrt{a x_{2}+b} J_{\frac{1}{3}}\left[\frac{2 \beta}{3 a}\left(a x_{2}+b\right)^{\frac{2}{2}}\right]
$$

The Bessel solution of the second kind was omitted because it leads to infinite values. In view of the above, an elementary solution of equation (4.4) would be:

$$
\bar{T}\left(x_{1}, x_{2}\right)=A \exp \left[\int_{0}^{x_{1}}-\beta^{2} D_{22}\left(x_{1}\right) d x_{1}\right] B \sqrt{a x_{2}+b} J_{\frac{1}{3}}\left[\frac{2 \beta}{3 a}\left(a x_{2}+b\right)^{\frac{3}{2}}\right]
$$

where $\mathrm{A}, \mathrm{B}$, and $\beta$ are constants. The most interesting remark about this form of the solution is that the rate of shear plays a definite role in diffusion of temperature across the thermal mixing layer. Solution (4.30) cannot satisfy the boundary conditions of the problem. However, this can be done by superimposing all elementary solutions in form of a series or an integral, where $\beta$ is a variable. This will be left for future work.

In addition to the analytical solution, one could also formulate a discret model for equation (4.4) and pursue a numerical solution of this problem. This is also left for future work. 


\section{Chapter 5}

\section{Experimental Apparatus and Measurement Procedure}

\subsection{The Flow Facility}

The wind-tunnel (fig. 5.1), where the experiments were conducted, was designed and constructed at the University of Ottawa and has been described by Iiarnik(1983). The facility was, however, modified by replacing the centrifugal fans with a mixed flow fan (Woods, model 63MIX, $630 \mathrm{~mm}$ diameter supplied with a $5 \mathrm{hp}$ motor). The flow of air was filtered by filters (Farr, model KB-HIE-40) and passed through a diffuser, a settling chamber with turbulence reducing screens and a 16:1 contraction. A shear generator was installed immediately following the contraction (fig. 5.2). The shear generator comprised a set of 12 separate channels, each 25.4 mm high, separated by aluminum plates, about $150 \mathrm{~mm}$ long. Screens of different resistances were attached across each channel so as to produce a uniform shear. A flow separator, consisting of 12 parallel plates, $610 \mathrm{~mm}$ long, aligned with those of the shear generator, was inserted into the flow in order to make the larger scales of 
the flow uniform on the transverse plane. The test section was $205 \mathrm{~nm}$ high, $4 \overline{5} \mathrm{i}$ $\mathrm{mm}$ wide and $5150 \mathrm{~mm}$ long. It provided 4 slots for the insertion of grids and other devices normal to the flow direction. The side walls of the second half of the test section diverged slightly to compensate for boundary layer growth.

\subsection{Heating System}

The heating system, shown in fig. 5.3, consisted of a wooden frame that could be inserted normal to the flow into the slots provided in the wind tunnel test section. Across the vertical walls of the frame, 23 heating elements (toaster elements) were stretched. The heating elements were $0.8 \mathrm{~mm}$ wide, $0.08 \mathrm{~mm}$ thick and $12.7 \mathrm{~mm}$ apart and were heated separately by variable voltage sources to produce the desired initial temperature profile. The heating elements were kept stretched using springs attached to their ends. The heating system was introduced into the flow at $x_{1}=$ 4.6h (fig. 5.4). At that position the turbulent stresses were nearly uniform in the transverse direction and grew exponentially in the streamwise direction (Tavoularis and Karnik, 1989).

\subsection{Calibration Jet}

The calibration jet, shown in fig. 5.5, was designed and constructed at the University of Ottawa for hot-wire, thermistor and cold-wire calibrations. A centrifugal blower produced air flow that passed through various turbulence reducing screens, a settling chamber and finally through a $24: 1$ nozzle with a $22.5 \mathrm{~mm}$ di- 
ameter, in order to generate a uniform jet. The air at the exit of the blower was heated by electric heaiers for temperature probe calibration. These probes could be mounted on a swivelling mechanism at any desired angle w.r.t. the axis of the jet.

\subsection{Velocity Measurements}

\subsubsection{The Hot Wire Anemometry}

The present measurements were conducted using a three-wire, specially made probe. This probe included 2 cross wires made up of tungsten wire and having diameters of $5 \mu \mathrm{m}$ and inclined at $\pm 45^{\circ}$ with respect to the probe body, as well as a cold wire (platinum wire) having a diameter of $1 \mu \mathrm{m}$ and a length of $0.4 \mathrm{~mm}$. The hot wires were powered by constant temperature bridges (TSI $1050 \mathrm{~A}$ with a TSI 1051 2-D monitor and power supply).

\subsubsection{Calibration}

Hot wire anemometry has been used extensively in measurements of mean velocities as well as the velocity fluctuations in turbulent flows. A hot wire is a thin cylinder, which is heated electrically. In the presence of a fluid flow, the rate of heat transfer from the wire varies in response to changes of the instantaneous velocity of the flow. This can be related to the instantaneous changes of voltage across the hot wire through the well known "King's law". A modified semi-empirical form 
of King's law is

$$
E^{2}=\left(A+B U^{n}\right)\left(T_{w}-T_{\mathrm{a}}\right)
$$

where $T_{w}, T_{a}$ are the temperatures of the wire and of the flow respectively, $\mathrm{U}$ is the flow velocity normal to the wire and $A, B$ and $n$ are constants to be determined from calibration. In the constant temperature mode, $T_{w}$ is maintained constunt by a feedback circuit. In the cross wire arrangement, where both wires are inclined at $\pm 45^{\circ}$ with respect to the flow and are perpendicular to each other, the effective cooling velocity, $U_{\text {eff }}$, defined as the hypothetical flow velocity normal to the wire that would produce the same cooling as the actual flow velocity, is given by

$$
\begin{aligned}
& U_{e f f 1}=\left(\overline{U_{1}}+u_{1}+\overline{U_{2}}+u_{2}\right) \cos 45^{\circ} \\
& U_{e f f 2}=\left(\overline{U_{1}}+u_{1}-\overline{U_{2}}-u_{2}\right) \cos 45^{\circ}
\end{aligned}
$$

Therefore, the modified King's law for the two wires can be written as

$$
\begin{aligned}
& \frac{E_{1}^{2}}{T_{w_{1}}-T_{a}}=A_{1}+B_{1} U_{e f \delta 1}^{n_{1}} \\
& \frac{E_{2}^{2}}{T_{w_{2}}-T_{a}}=A_{2}+B_{2} U_{e f \delta 2}^{n_{2}}
\end{aligned}
$$

Because in the present work the air flow is not isothermal, corrections for the temperature sensitivity of the hot wires were made by measuring the instantaneous temperature of the flow at each measuring station and using equation (5.4) and equation (5.5) as

$$
\begin{aligned}
& U_{e f \delta 1}=\left[\frac{\left(\frac{E_{1}^{2}}{T_{w_{1}}-T_{a}}\right)-A_{1}}{B_{1}}\right]^{\frac{1}{n_{1}}} \\
& U_{e f \delta 2}=\left[\frac{\left(\frac{E_{2}^{2}}{T_{w_{2}}-T_{a}}\right)-A_{2}}{B_{2}}\right]^{\frac{1}{n_{2}}}
\end{aligned}
$$


Finally from equation (5.2) and equation (5.3) the mean and fluctuation velocities were computed as

$$
\begin{gathered}
\overline{U_{1}}=\frac{\bar{U}_{e f f 1}+\bar{U}_{e f f 2}}{2} \\
\overline{U_{2}}=\frac{\bar{U}_{e f f 1}-\bar{U}_{e f f 2}}{2} \\
u_{1}=\frac{U_{e f f 1}-\bar{U}_{e f f 2}}{2}-\overline{U_{1}} \\
u_{2}=\frac{U_{e f f 1}-U_{e f f 2}}{2}-\overline{U_{2}}
\end{gathered}
$$

The hot wires were calibrated against a pitot tube in the wind tunnel with all obstructions removed to provide a low turbulence air stream $\left(\frac{u^{\prime}}{U} \approx 005\right)$. The hot wires were positioned in the proximity of the pitot tube. The pressure differences were measured with a pressure transducer that was calibrated against a Meriam micromanometer. Typical calibration curves for the pressure transducer and the hot wires are shown in fig. 5.6 , and fig. 5.7 respectively.

\subsection{Temperature Measurements}

\subsubsection{Mean Temperature}

Thermistors were used as best suited for measurements of mean temperature of the flow. These were calibrated in the heated jet at temperatures ranging from $21^{\circ} \mathrm{C}$ to $44^{\circ} \mathrm{C}$ versus a mercury thermometer with $0.1^{\circ} \mathrm{C}$ resolution. The mean

- temperature of the flow was measured with glass-coated, thermistor mini-probes

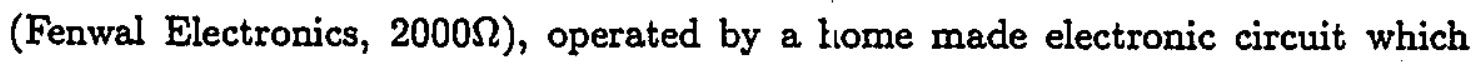
could provide individual signals as well as direct measurements of the temperature difference by subtracting the conditioned outputs of the thermistors (fig. 5.8). The 
ambient temperature was monitored upstream of the heating elements. Typical calibration curves for thermistors are shown in fig. 5.9.

\subsubsection{Temperature Fluctuations}

The temperature fluctuations were measured with a cold wire mounted on the same probe as the cross wires. The cold wire was calibrated in the calibration jet against a previously calibrated thermistor. The cold wire output powered by a constant current circuit (fig. 5.10), was calibrated at a gain of 250 and its output was low-pass filtered in order to reduce noise. A typical calibration curve for the cold wire is shown in (fig. 5.11.)

Measurements of temperature fluctuations were recorded at a gain of 50000 . The signal was low-pass filtered at $3 \mathrm{KHz}$ to eliminate the high frequency noise. A current of about $0.5 \mathrm{~mA}$ was used. At this current level, the velocity sensitivity of the cold-wire measured with the wire placed in the unheated flow at different speeds was found negligible. The r.m.s. output of the cold-wire in the heated flow was very large compared to that in the case of the unheated flow (termed as noise) which was $e_{n}^{\prime} \approx 0.017^{\circ} \mathrm{C}$. Assuming that the noise was statistically independent of the temperature fluctuation, a correction was applied to the cold-wire signal to eliminate noise contamination. 


\subsection{Signal Conditioning and Data Acquisition}

All signals were digitized by a Data Translation board,(DT2828), mounted on an IBM-PC-AT compatible personal computer (system 1800, 20 MB random Access Memory, $60 \mathrm{MB}$ hard disk drive). The DT2828 is a 12-bit analog to digital converter with a $\pm 10 \mathrm{~V}$ range, giving a $5 \mathrm{mV}$ nominal resolution and simultaneous sampling of up to four channel inputs.

The hot wire anemometer signals were conditioned for digitization using the electronic circuit shown in fig. 5.12. This circuit could offset the input signals by a $\mathrm{DC}$ voltage between $\pm 7 \mathrm{~V}$, amplify the signals by a gain of $.5,1 ., 2.0,10.0,20.0$ or 50.0 and low pass filter the amplified signals with cut-off frequencies of 1,3 , or $10 \mathrm{KHz}$. Proper choice of the offset voltage and gain provided output values that optimized the resolution of the analog to digital converter.

The cold wire outputs were observed on a Tektronix model 5113 dual beam storage oscilloscope. The oscilloscope has two differential amplifiers (Tektronix 5A22A) with gains ranging from 0.1 to 50000 and built-in high pass and low-pass filters with $3 \mathrm{db}$ drop-off per octave.

The signals generated from the cross-wire and the cold-wire were digitized and sampled simultaneously at two different frequencies, $25 \mathrm{KHz}$ and $2 \mathrm{KHz}$, using 40 records and 2048 points for each signal and each frequency. The time intervals between two consecutive points was $40 \mu \mathrm{s}$ and $0.5 \mathrm{~ms}$ respectively and the sample time for each record was therefore $82 \mathrm{~ms}$ and 1 second which were at least 100 times

the temporal microscale (i.e. $\frac{\lambda_{11}}{U_{c}}$; typically $0.8 \mathrm{~ms}$ ) and the integral time scale of the flow (typically $6 \mathrm{~ms}$ ) respectively. The high frequency records were used to evaluate 
the microscales, while the low frequency ones were used to evaluate all moments and integral length scales. 


\section{Chapter 6}

\section{Measurements}

\subsection{Velocity Measurements}

\subsubsection{Free Stream Measurements}

As mentioned in Chapter 4, new fans and controls were installed in the windtunnel. For this reason it was felt necessary to conduct measurements in the empty tunnel. Measurements of the free stream velocity, as indicated in fig. 6.1 , show that the centerline velocity was almost constant at mean speeds ranging from $5 \mathrm{~m} / \mathrm{s}$ to about $20 \mathrm{~m} / \mathrm{s}$. The turbulence intensity on the centerline was less than $0.5 \%$ (fig. $6.2)$.

\subsubsection{The Mean Velocity Field}

Measurements of mean. velocity at a centerline speed of about $5 \mathrm{~m} / \mathrm{s}$ and at different locations are shown in fig. 6.3. These measurements showed that the mean velocity profiles exhibited a good linearity except at high downstream distances 
where the effect of boundary layers was seen. In any case, this effect was outside the region of interest for the thermal mixing layer growth. The shear parameter, $\beta$, for the above mentioned speed was about

$$
\beta=\frac{h}{\bar{U}_{c}} \frac{d \bar{U}_{1}}{d x_{2}} \approx 2.05
$$

which is the same as the value found by Karnik and Tavoularis (1987); $h=0.305 \mathrm{~m}$ is the wind tunnel height.

\subsubsection{Reynolds Stresses}

$\overline{u_{1}^{2}}$ and $\overline{u_{2}^{2}}$ were the only normal stresses measured. The observed transverse variations in $\frac{u_{1}^{\prime}}{U_{c}}$ and $\frac{u_{2}^{\prime}}{U_{c}}$ (fig. 6.4) were found to be comparable to those in earlier reported measurements. The inhomogeinity of the flow was found to be $25 \%$ at its worst in the core of the tunnel. Fig. 6.5 shows that the shear stress correlation coefficient $\rho=\frac{\overline{u_{i} u_{3}}}{u_{1} u_{2}}$ reached an asymptotic value, about -0.45 , comparable to values reported by Tavoularis and Karnik (1989).

Exponential fitting to the ratio $\frac{\overline{u_{i} w_{i}}}{U_{c}^{2}}, i=1,2$, gave the relations

$$
\overline{\overline{u_{1}^{2}}} \overline{\overline{U_{c}^{2}}}=0.01034 e^{0.015 \frac{x_{1}}{M}}
$$

and

$$
\frac{\overline{u_{2}^{2}}}{\overline{U_{c}^{2}}}=0.0015 e^{0.015 \frac{x_{1}}{M}}
$$

Plots of these ratios along with the results of Tavoularis and Karnik (1989) are presented in fig. 6.6, showing that the growth rates of these quantities in the two experiments were comparable. 


\subsubsection{Integral Length Scales and Microscales.}

A few measurements of the integral length scales $L_{11,1}, L_{22,1}$ were plotted in fig. 6.7. Exponential fitting to $L_{11}$ was found to be

$$
L_{11}=0.014 e^{0.012 \frac{x_{1}}{N}}
$$

Semilogarithimic plots of $L_{11}$ found in this experiment and measured by Tavoularis and Karnik (1989) are shown in fig. 6.8. These measurements seem to be of the same order of magnitude, although in this experiment, $L_{11}$ exhibit somewhat lower values. It seems that the passage of the flow through the ribbon screen has resulted in a reduction of eddy sizes and, thus, of integral length scales. Another possible cause for the discrepancy is the difference in the center-line speeds in the two experiments. Measurements of the Taylor microscales $\lambda_{11}$ and $\lambda_{22}$ are presented in fig. 6.9. These demonstrate that $\lambda_{11}$ was roughly constant along the centerline (see also fig. 6.10).

\subsection{Temperature Measurements}

\subsubsection{Initial Temperature Field}

The heating system has been described in section 5.2. To produce a stepwise mean temperature profile, only the ribbons in the upper half of the tunnel were heated at rates ranging from $85 \Omega / m$ to $350 \Omega / m$.

A major concern when using this technique was the possible distortion of the velocity field by the physical presence of the heating elements or heating itself. 
Profiles of the velocity field downstream of the heating ribbons (fig. 6.11) show that, at the position $\frac{x_{1}}{M}=16$, the effect of the presence of heating elements on the mean velocity profile was essentially unnoticeable. At that location the upper half of the wind-tunnel was at a uniform temperature of $1.12^{\circ} \mathrm{K}^{\circ}$ above the ambient temperature $\left(\bar{T}_{m}=1.12^{\circ} K^{\circ}\right)$, as shown in Fig. 6.12a.

\subsubsection{Mean Temperature}

Measurements of the mean temperature rise at different stations (up to $\frac{x_{1}}{M}=$ 105) are presented in fig. 6.12. Fig. 6.13 is a plot of lines of constant mean temperature (isotherms), showing that mixing layer centerline of the mixing layer was shifted towards the low velocity region and that the mixing layer penetrated deeper in the low velocity side than in the high velocity side. A similar observation has been made by Karnik and Tavoularis (1989) in the case of diffusion of heat

from a line source in uniformly sheared, turbulent flow. The growth of the width of the mixing layer, defined in this case as the distance between isotherms at $75 \% \bar{T}_{m}$ and $25 \% \bar{T}_{m}$, is shown in fig. 6.14 . The growth rate seems to be roughly constant.

\subsubsection{Temperature Fluctuations}

The transverse distribution of the temperature variance, $\overline{\theta^{2}}$, is shown in fig. 6.15. All profiles exhibited a peak of temperature variance near the center of the thermal mixing layer. However, close to the heating screen, high temperature variances were also found in the heated half of the flow, due to the mixing of the warm wakes of the ribbons with the cool streams around them. Further downstream, 
those fluctuations died out but the central peak of the temperature variance persists, due to the production of $\overline{\theta^{2}}$ by the mean temperature gradient, $\frac{d \bar{T}}{d x_{2}}$. The locations of the maximum temperature variance are plotted in fig. 6.16. Like the center of the thermal mixing layer, temperature variance peals also were shifted towards the low velocity side of the wind tunnel.

Measurements of the skewness factor of the temperature fluctuations are presented in fig. 6.17. This factor had a positive peak at the cooler half of the thermal mixing layer and a negative peak at the warmer half of the mixing layer.

\subsubsection{Temperature-Velocity Correlations.}

Transverse profiles of the correlation coefficients $\rho_{u_{1} \theta}=\frac{\overline{\overline{u_{1} \theta}}}{u_{1}^{\prime} \theta^{\prime}}$ and $\rho_{u_{2} \theta}=\frac{\overline{u_{2} \theta}}{u_{2}^{\prime} \theta^{\prime}}$ are shown in fig. 6.18 and fig. 6.19 respectively. $\rho_{u_{1} \theta}$ was always positive and peaked at the center of the mixing layer, whereas $\rho_{u_{2} \theta}$ was always negative and also peaked near the center of the mixing layer. Centerline measurements of $\rho_{u_{1} \theta}$ and $\rho_{u_{2} \theta}$, shown in fig. 6.20 and fig. 6.21 respectively, seem to reach asymptotically constant values near 0.55 and -0.48 respectively.

Fig. 6.22 and fig. 6.23 show the transverse distributions of the triple correlation coefficients $\frac{\overline{u_{1} \theta^{2}}}{u_{1}^{\prime} \overline{\theta^{2}}}$, and $\frac{\overline{u_{2} \theta^{2}}}{\frac{u_{2}^{\prime}}{\theta^{2}}}$ respectively. $\frac{\overline{u_{1} \theta^{2}}}{u_{1}^{\prime} \bar{\theta}^{2}}$ seems to have two peaks of opposite signs growing in magnitude with downstream distances. The positive peak was located in the low temperature side, whereas the negative peak was located in the high temperature side of the wind tunnel. $\frac{\overline{\frac{3}{2} \theta^{2}}}{u_{2}^{\prime} \theta^{2}}$ showed the same trend as $\frac{\overline{u_{1} \theta^{2}}}{\overline{u_{1}^{\prime}} \cdot \overline{\theta^{2}}}$ but it was of the opposite sign. 


\section{Chapter 7}

\section{Analysis and Discussion of Results}

\subsection{Similarity of the Mean Temperature Profiles}

The mean temperature profiles appeared to have shapes not very different from that of an error function. As an approximation, therefore, an error function was fitted to the data for each profile by matching the corresponding points with $25 \%$ and $75 \% \overline{T_{m}}$. These fitted functions were differentiated analytically to provide the maximum temperature gradient, $\frac{\partial\left(\frac{\bar{T}}{T_{m}}\right)}{\partial\left(\frac{T_{M}}{M}\right)}$, at the center of the layer, which has been plotted versus $\frac{x_{1}}{M}$ in fig. 7.1. This quantity decreased with increasing downstrean distance due to the growth of the width of the thermal mixing layer. However, if one normalizes the transverse coordinate, $x_{2}$, with the mixing layer width, $\omega$, the maximum temperature gradient, $\frac{\partial\left(\frac{\bar{T}}{T_{m}}\right)}{\partial\left(\frac{T_{2}}{\omega}\right)}$ attains a nearly constant value, close to 0.5 , (fig. 7.2). Furthermore, mean temperature profiles plotted against $\frac{ \pm_{2}}{w}$ (fig. 7.3), nearly collapse, implying a quasi-self-similar development of the mean temperature field. A closer examination of fig. 7.3, however, reveals a systematic deviation of these profiles from the error function, especially at their two fnds, which is another 
manifestation of the asymmetry of the mixing layer caused by the shear.

\subsection{Turbulent Diffusivity}

Direct measurements of the turbulent diffusivity, $D_{22}$, at the inflection point of the mean temperature profiles are shown in fig. 7.4. Other estimates of this quantity from triple correlations (Karnik and Tavoularis, 1989) at the maximum

gradient of the temperature fluctuations $\left(D_{22}=-\frac{\overline{\theta^{2} u_{2}}}{\frac{\partial \theta_{2}^{2}}{\partial 5_{2}}}\right)$ are also shown in fig. 7.4. The exponential curve

$$
D_{22}=9.31 \times 10^{-4} e^{0.014 \frac{\pi 1}{14}}
$$

seems to fit well to all measurements. It has been suggested that the turbulent diffusivity can be expressed as the product of a Lagrangian characteristic velocity and a Lagrangian characteristic length. In homogeneous flows, this quantity can also be estimated from the product of the corresponding Eulerian scales. The ratio $\frac{D_{22}}{u_{2}^{\prime} L_{11,1}}$, shown in fig. 7.5 , was fairly constant, except close to the heaters. Therefore, one may conclude that the gradient transport model models this type of flow. Similar conclusions for the uniformly sheared turbulence have been reached by Sreenivasan et al. (1982), Tavoularis and Corrsin (1981) and Karnik and Tavoularis (1989).

\subsection{Comparison with the Approximate Solution}

The approximate self-similar solution, developed in section 4.2 and based on the assumption that the mean velocity field was constant across a narrow region of 
the thermal mixing layer, is given by

$$
\frac{\bar{T}(\eta)}{\bar{T}_{m}}=\frac{1}{2}[\operatorname{erf}(\sqrt{\pi}(\eta-\beta))+1]
$$

where

$$
\eta=\frac{x_{2}}{g\left(x_{1}\right)}+\beta
$$

and the scaling function is given by

$$
g\left(x_{1}\right)=\left[\frac{4 \pi D_{0}}{\overline{U_{c}} k_{D}}\left(e^{k_{D} x_{1}}-1\right)+A\right]^{\frac{1}{2}}
$$

The width of the mixing layer was defined as the distance separating the isotherms of $75 \% \bar{T}_{m}$ and $25 \% \bar{T}_{m}$. Therefore, one can write

$$
\left(\eta_{75 \%}-\beta\right)-\left(\eta_{i 5 \%}-\beta\right)=\frac{x_{23 \%}-x_{235 \%}}{g}=\frac{\omega}{g}
$$

On the other hand, equation (7.1) yields

$$
\frac{1}{2}\left[\operatorname{erf}\left(\sqrt{\pi}(\eta-\beta)_{75 \%}\right)+1\right]=0.75 \Rightarrow(\eta-\beta)_{75 \%}=\frac{0.48}{\sqrt{\pi}}
$$

and

$$
\frac{1}{2}\left[\operatorname{erf}\left(\sqrt{\pi}(\eta-\beta)_{25 \%}\right)+1\right]=0.25 \Rightarrow(\eta-\beta)_{75 \%}=\frac{-0.4 \$}{\sqrt{\pi}}
$$

which, if substituted in equation (7.4), yields

$$
\omega\left(x_{1}\right)=\sqrt{\frac{0.98}{\pi}} g\left(x_{1}\right)
$$

or

$$
\omega\left(x_{1}\right)=\left[\frac{3.98 D_{0}}{\overline{U_{c}} k_{D}}\left(e^{k_{D} x_{1}}-1\right)+A\right]^{\frac{1}{2}}
$$

where $D_{o}, k_{D}$ and $A$ are to be found from experimental data.

From the measurements of $D_{22}$, shown in fig. $7.4, D_{o}$ and $k_{D}$ were fcund to 
be $9.31 \times 10^{-4} \mathrm{~m}^{2} / \mathrm{s}$ and $0.55 \mathrm{~m}^{-1}$ respectively. The constant $A$ in equation (7.6) was found by matching the experimental value of $\omega$ at $\frac{x_{1}}{M}=40$ to be $-4.28 \times$ $10^{-4} \mathrm{~m}^{2}$. Substituting these constants in equation (7.6), one gets an expression for the thermal mixing layer width as

$$
\omega\left(x_{1}\right)=0.036\left[12.9 e^{0.014 \frac{x_{1}}{M}}-1.33\right]^{\frac{1}{2}}
$$

It is remarkable that, although based on the assumption that the mean velocity was constant across the thermal mixing layer, this solution seems to agree well with the measurements of the width (fig. 7.6).

Substituting equation (7.7) in equation (7.2), one may get the dimensionless coordinate, $\eta$, as

$$
\eta=\frac{x_{2}}{0.0646\left[e^{0.014 \frac{x}{\mu}}-1.33\right]^{\frac{1}{2}}}+\beta
$$

All mean temperature profiles as well as the approximating solution (7.1) were plotted against the assumed similarity variable $\eta$ (fig. 7.6). The approximate solution seemed to fit well the mean temperature profiles except near their two ends. This fact was expected because the asymmetry caused by the mean shear was not considered in the analysis; it remains to be seen whether modifying the analysis to account for the shear will predict this asymmetry.

\subsection{Comparison with Isotropic Diffusion}

Plots of the mixing layer width for the present flow as well as for the isotropic case (LaRue et al., 1981) are presented in fig. 7.8. This figure reveals that the thermal mixing layer spreads faster in the present flow than in the isotropic case. 
This can be explained by the fact that the velocity and length scales, which are responsible for scalar transport and diffusion, grow at faster rates in uniformly sheared, turbulent flow than in the case of isotropic turbulence, where turbulence decays.

An argument that has been dealt with over many years is that thermal mixing layers in isotropic turbulence cannot be self-similar because the temperature and velocity length scales do not grow at the same rate (Ma and Warhaft, 1986). Shown in fig. 7.8 , the ratio $\frac{L_{\theta}}{L_{11,1}}$ appears to be constant confirming that, in the shear flow, the temperature and velocity length scales grow at the same rate. This was also observed by Karnik and Tavoularis (1989).

\subsection{Temperature Fluctuations}

An estimate of peak r.m.s. temperature fluctuations can be made based on the gradient transport model. Considering that, by definition,

$$
\overline{\theta u_{2}}=-D_{22} \frac{\partial \bar{T}}{\partial x_{2}}
$$

and

$$
\overline{\theta u_{2}}=\rho_{\theta u_{2}} u_{2}^{\prime} \theta^{\prime}
$$

and, as shown earlier,

$$
\frac{D_{22}}{u_{2}^{\prime} L_{11,1}} \approx D_{0} \approx \text { const }
$$

one may deduce that

$$
\overline{\theta u_{2}}=\rho_{\theta u_{2}} u_{2}^{\prime} \theta^{\prime}=D_{0} u_{2}^{\prime} L_{11,1} \frac{\partial \bar{T}}{\partial x_{2}}
$$


or

$$
\frac{\theta^{\prime}}{L_{11,1} \frac{\partial \bar{T}}{\partial x_{2}}}=\frac{D_{0}}{\rho_{\theta u_{2}}} \approx \text { constant }
$$

Measurements of this ratio, shown in fig. 7.10 , show its near constancy, except close to the heaters, where $\theta^{\prime}$ is affected by the wakes of the individual ribbons.

\subsection{Temperature Velocity Covariances}

In the present flow, it was found that $\rho_{\theta u_{2}}$ was always negative. This fact can be explained using a simple gradient transport model and considering that the mean temperature gradient is $\frac{\partial \bar{T}}{\partial x_{2}} \geq 0$. Because in the shear flow $\overline{u_{1} u_{2}}<0$, one would expect that $u_{1}$ has, on the average, a sign opposite to the sign of $u_{2}$. Thus $\rho_{\theta u_{1}}$ is expected to have a sign opposite to the sign of $\rho_{\theta u_{2}}$, i.e. positive.

For the case of triple correlations, one could consider that the transported quantity is $\theta^{2}$. Therefore, the sign of $\overline{\theta^{2} u_{2}}$ could be explained by applying the gradient transport model as (Irarnik and Tavoularis, 1989).

$$
\overline{\theta^{2} u_{2}}=-D_{i j} \frac{\partial \overline{\theta^{2}}}{x_{2}}
$$

Similarly, the sign of $\overline{\theta^{2} u_{1}}$ would be expected to be opposite to that of $\overline{\theta^{2} u_{2}}$, as indeed is observed.

The non-zero values of skewness factor $S_{\theta}$ are a consequence of asymmetry of the probability density function of the temperature fluctuations. Positive skewness implies that positive values of $\overline{\theta^{3}}$ are likely to have larger magnitudes but less frequent occurrences than negative values of $\overline{\theta^{3}}$. In the present study positive skewness factors were found in the bottom half of the thermal mixing layer, 
corresponding to a temperature field consisting of relatively small hot fludd eddies, embedded in a cold fluid background. In the upper half of the thermal mixing laver negative skewness factors were found, indicating that the inverse phenomenon is taking place. 


\section{Chapter 8}

\section{Conclusion}

The main conclusions of the present thesis could be summarized as follows:

a) Large turbulent fluctuations and fast growth of scales caused by the shear results in a spread of the temperature field faster than that in unsheared turbulence.

b) The shear caused the thermal mixing layer to penetrate deeper in the low velocity side than in the high velocity side, thus producing asymmetry of the mixing layer. c) To a first approximation, the mean temperature field approached a self similar state.

d) The gradient transport model applies well to this type of flows. 


\section{Bibliography}

[1] CHUNG, M.K. and KYONG, N.H. 1989 Measurements of turbulent dispersion behind a fine cylindrical heat source in weakly shenr flow. J. Fluid. Mech. 205, 171.

[2] CORRSIN, S. 1952 Heat transfer in isotropic turbulence. J. Appl. Phys. $\underline{23}, 113$.

[3] CORRSIN, S. 1977 Limitations of gradient transport models in random walks and in turbulence. Adv. Geophys. 18A, 25.

[4] ELGOBASHI, S.E. and LAUNDER, B.E. $19 S 3$ Turbulent time scales and the dissipation rate of the temperature varia.'ce in the thermal mixing layer. Phys. Fluids $\underline{26}, 2415$.

[5] ELRICK, D.E. 1962 Sourse functions for diffusion in uniform shent flow. Austr. J. Phys. 15, 283.

[6] FOX, J. 1964 Turbulent temperature fuctuation and two dimensional heat transjer in a uniform shear flow. NASA Tech. Note D2511.

[7] GIBSON, M.M. JONES, W.P. and KANELLOPOULOS, V.E. 1989 Turbulent temperature mixing layer: Measurements and modelling. In Turbulent Shear Flows.Vol.6 (ed. J.-C. André et al.). 119, Springer.

[8] HINZE, J.O. 1975 Turbulence, $2^{\text {nd }}$ Ed. McGraw-Hill. 
[9] HUANG, C. H. $1979 A$ theory of dispersion in turbulent shear flow. Atmos. Environ. 13, 453.

[10] KARNIK, U. 1988 Experiments on Turbulent Structure and Heat Diffusion from a Line Source in Uniformly Sheared Turbulent Flows. $\mathrm{Ph} . \mathrm{D}$ Thesis, Department of Mechanical Engineering, University of Ottawa.

[11] KARNIK, U. and TAVOULARIS, S. 1989 Mesurements of heat diffusion from a continous line source in uniformly sheared turbulent flow. J. Fluid Mech. 202, 233.

[12] KEFFER, J. F. OLSEN, G. J. and KAWALL, J. G. 1977 Intermittency in a thermal mixing layer. J. Fluid. Mech. 79, 595.

[13] LARUE, J.C, LIBBY, P.A. 1981 Thermal mixing layer downstrcam of a half-heated turbulence grid. Phys. Fluids 24, 597.

[14] LARUE, J.C, LIBBY, P.A., SESHADRI, D.V.R. 1981 Further results on thermal mixing layer downstream of a half-heated turbulence grid. Phys. Fluids 24, 1927.

[15] LIBBY, P.A 1975 Diffusion of heat downstream of a half-heated turbulence grid. Acta Astron. 2,867 .

[16] LUMLEY, J.L. 1986 Evolution of non-self-preserving mixing layer. Phys. Fluids 29, 3976.

[17] MA, B.K. and WARHAFT, Z. 1986 Some aspect of the thermal mixing layer in grid turbulence. Phys. Fluids 29, 3114.

[18] MONIN, A.S. and YAGLOM, A.M. 1971 Statistical Fluid Mechanics. M.I.T Press. 
[19] NAKAMURA, I. , SAKAI, Y., MIYATA, M. , and TSUNODA. H. 1980 Diffusion of natter from a continuous point source in uniform meian shear flows., Bull. JSME. 22, 1141.

[20] NOVIKOV, E.A 1958 Turbulent diffusion in shear flow. Prikl. Matem. Mekh. 22, 412.

[21] OKUBO, A. and KARWEIT, M.J. 1969 Diffusion from a continutous source in a uniform shear flow Limnol. Oceanogr. 14, 514.

[22] RILEY, J. and CORRSIN, S. 1971 Simulation and computation of dis.persion in turbulent shear flow. Proc. Conf. on Air Pollut. Met., Amer. Met. Soc. 16.

[23] RILEY, J. and CORRSIN, S. 1974 The relntion of turbulent duffusivities to Lagrangian statistics for the simplest shear flow. J. Gcopl.. Res. ID, 1768.

[24] ROGERS, M.M., MANSOUR, N.N. and REYNOLDS, W.C. $10 S 9$ An algebraic model for the turbulent fiux of a passive scalar. J. Fluid Mech. $\underline{203}, 77$.

[25] SAIAI, Y., NAKAMURA, I. and TSUNODA, H. $10 S 6$ Diffusion. of matter from a continuous point source in uniform mean shenr flow. Bull. JSME. 29, 1149.

[26] SREENIVASAN, K.R, TAVOURARIS, S. and CORRSIN, S. 19S2 A test of gradient transport and its generalizations. In Turbulent Shcar Flows, vol. 3 (ed. L.J.S. Bradbury et al.). 96, Springer.

[27] STAPOUNTZIS, H. and BRITTER, R.E. 1989 Turbulent diffusion behind a heated line source in a nearly homogeneous turbulent shent flow. In Turbulent Shear Flows, vol. 6 (ed. J.C. André et al.). 07, Springer. 
[28] TAVOULARIS, S. 1985 Asymptotic laws for transversely homogeneous turbulent shear flows. Phys. Fluids $\underline{2 \&,} 999$.

[29] TAVOULARIS, S. and CORRSIN, S. 19S1 Experiments in a nearly homogeneous shear flow with a uniform mean temperature gradient, part 1. J. Fluid Mech. 104, 311.

[30] TAVOULARIS, S. and CORRSIN, S. 1985 Effects of shear on the turbulent diffusivity tensor. Int. J. Heat Mass Transfer $2 S, 265$.

[31] TAVOULARIS, S. and KARNIK, U. 1989 Further experiments on the evolution of turbulent stresses and scales in uniformly sheared turbulence. J. Fluid Mech. 204, 457.

[32] TAYLOR, G. I. 1921 Diffusion by continuous movements. Proc. Lond. Math. Soc. A20, 196.

[33] WATT, W.E. and BAINES W.D. 1973 The turbulent temperature mixing layer. J. Hydr. Res 11, 157. 


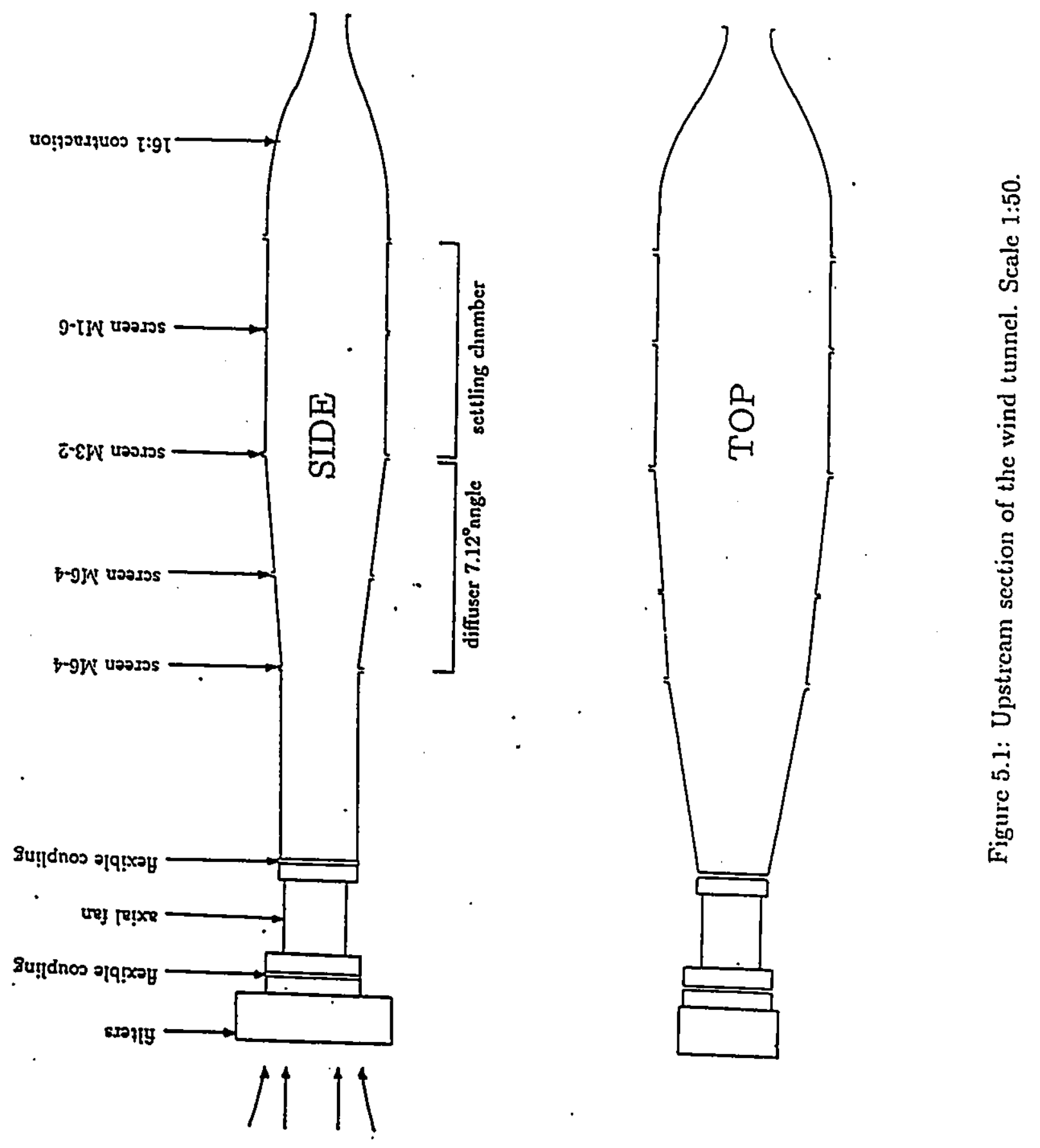



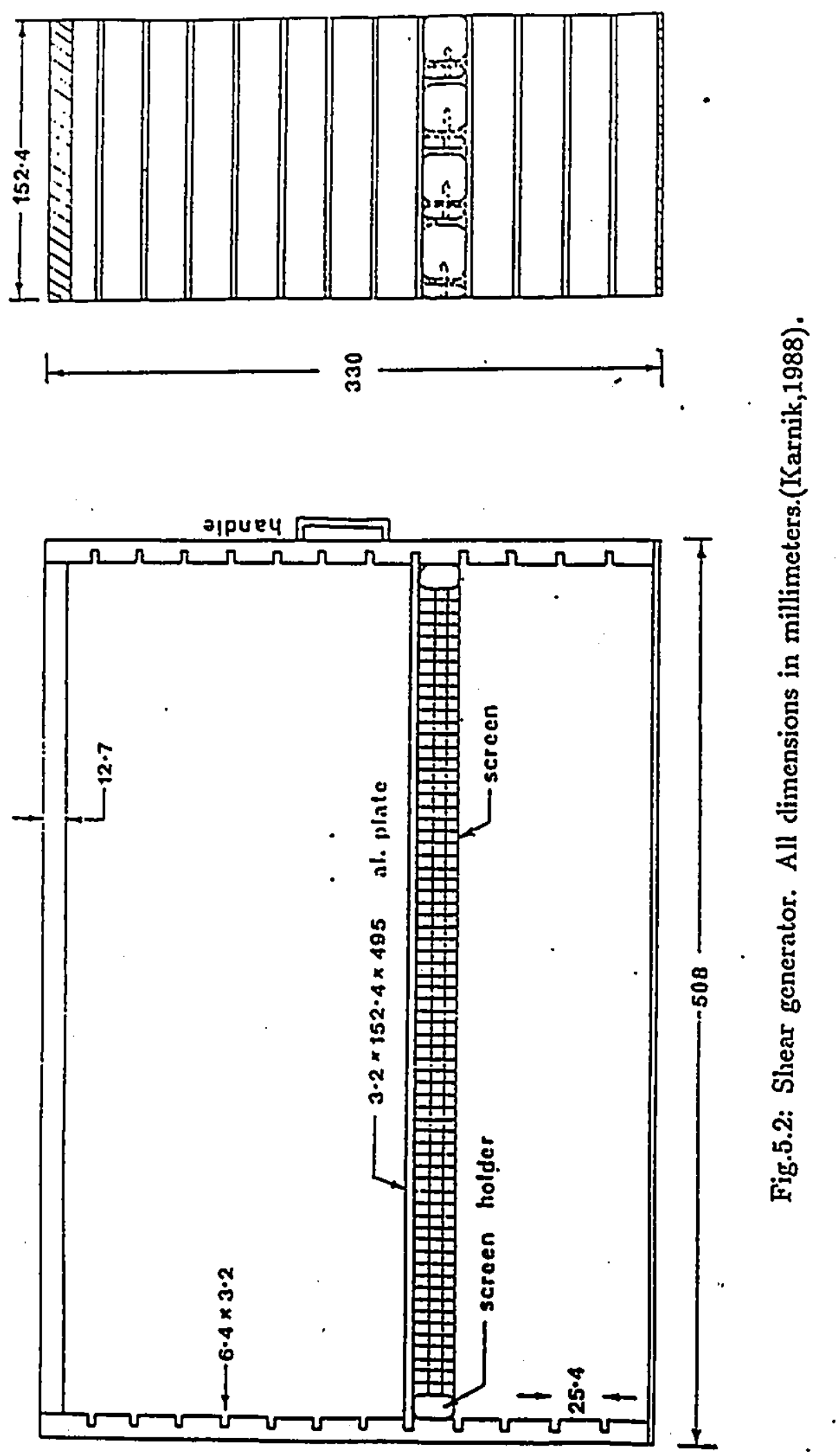


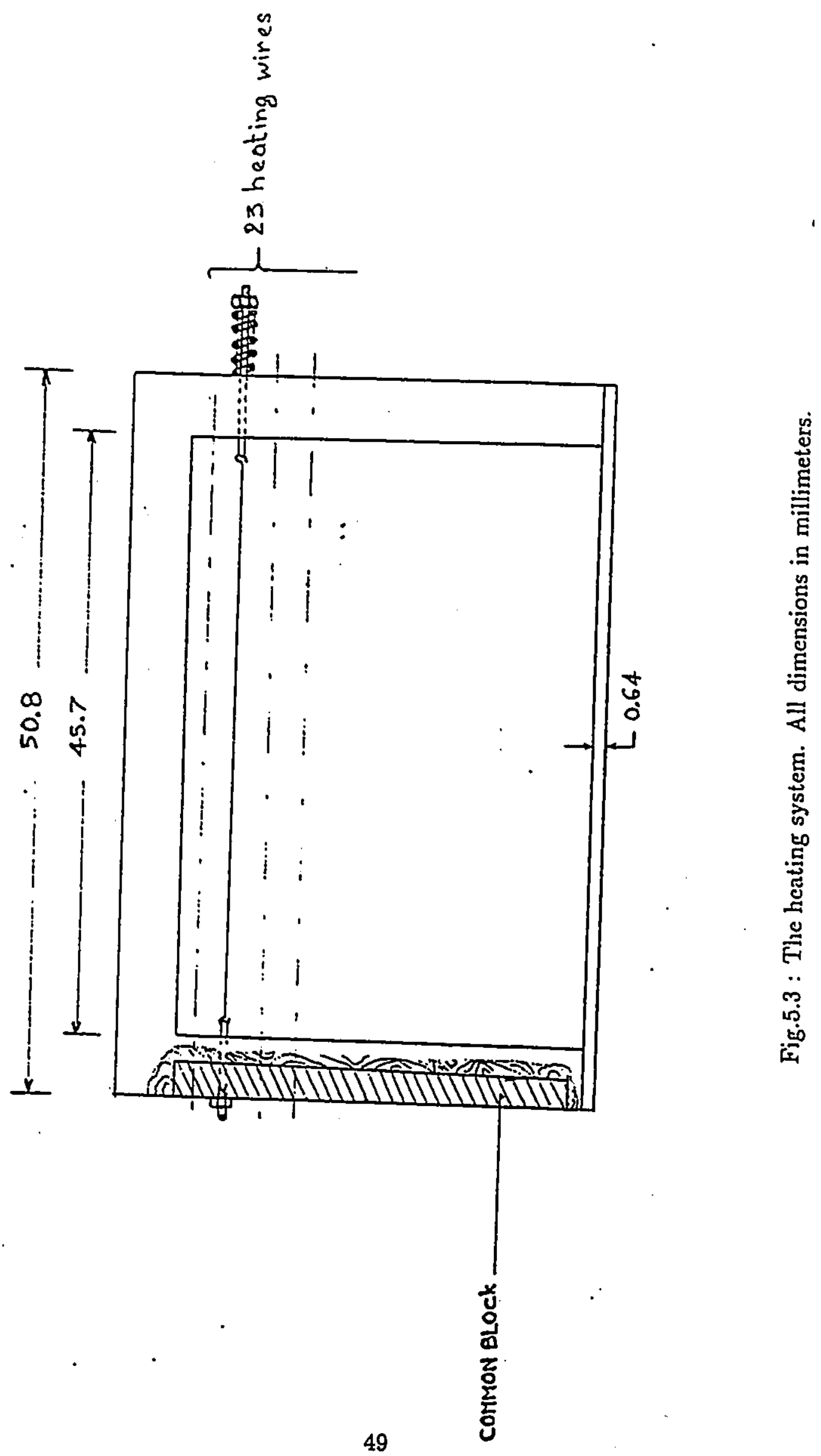




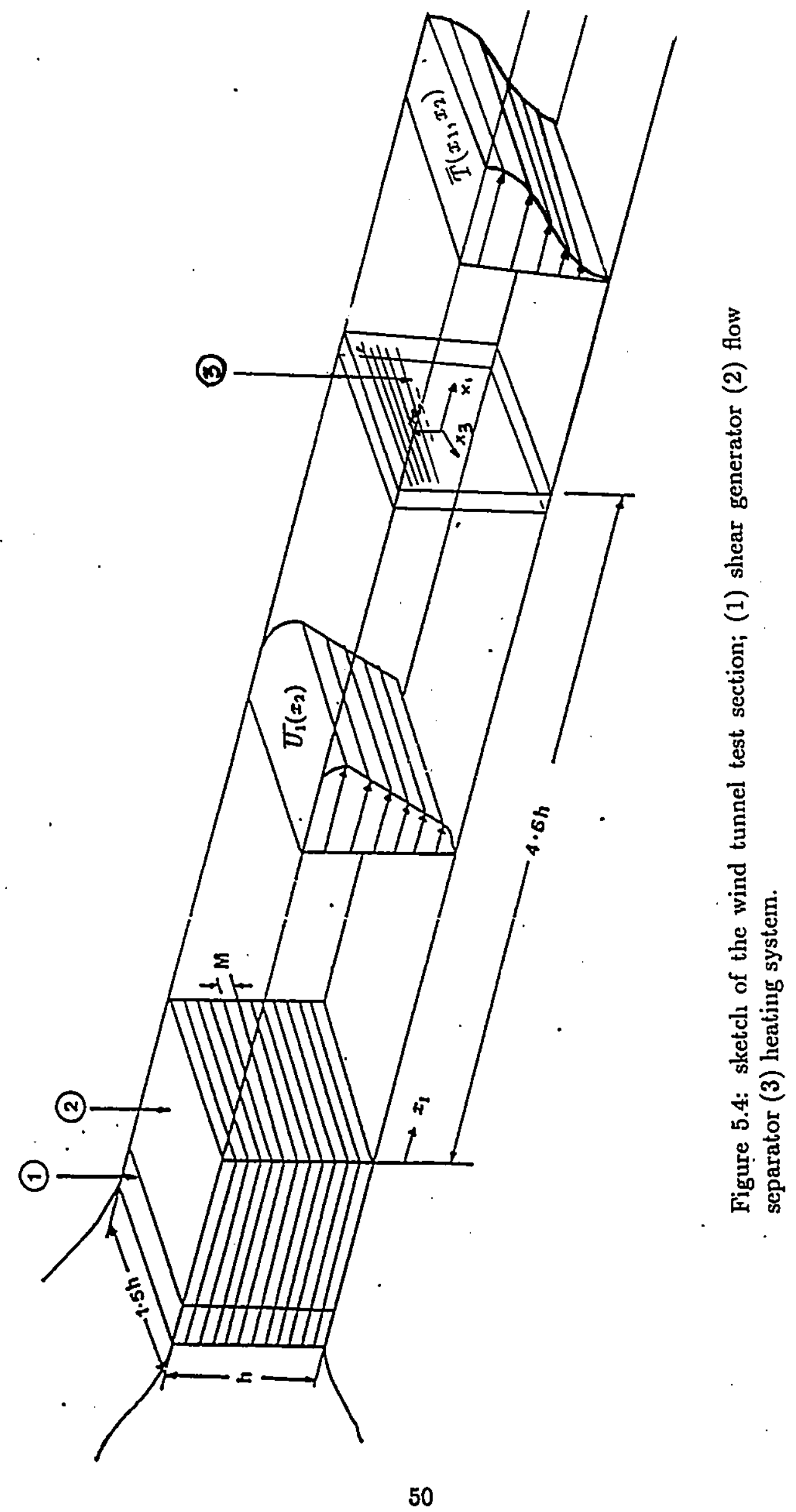




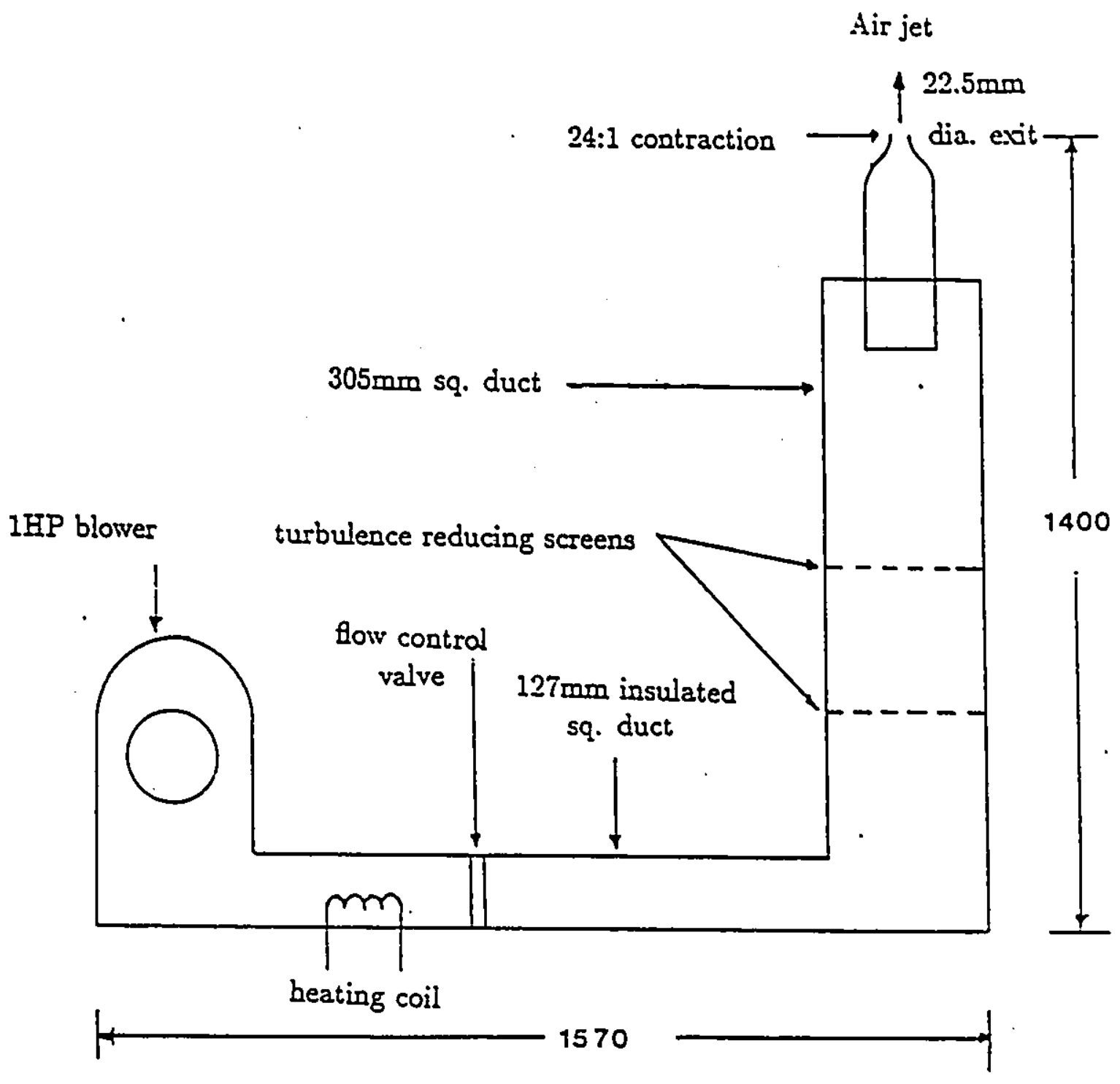

Figure 5.5: The calibration jet. All dimensions in millimeters (Karnik, 1988). 


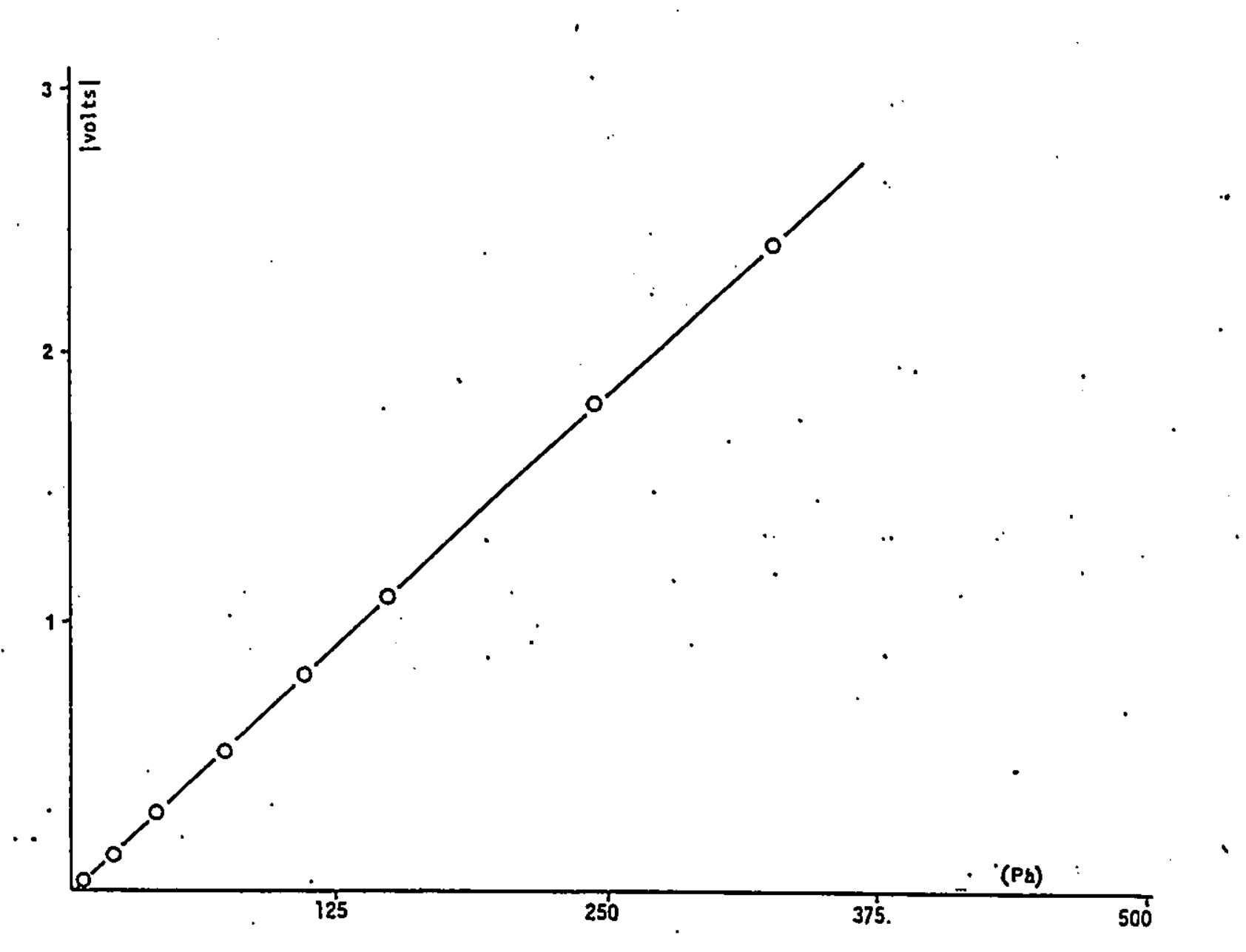

Figure 5.6: Typical calibration curve for the pressure transducer. 


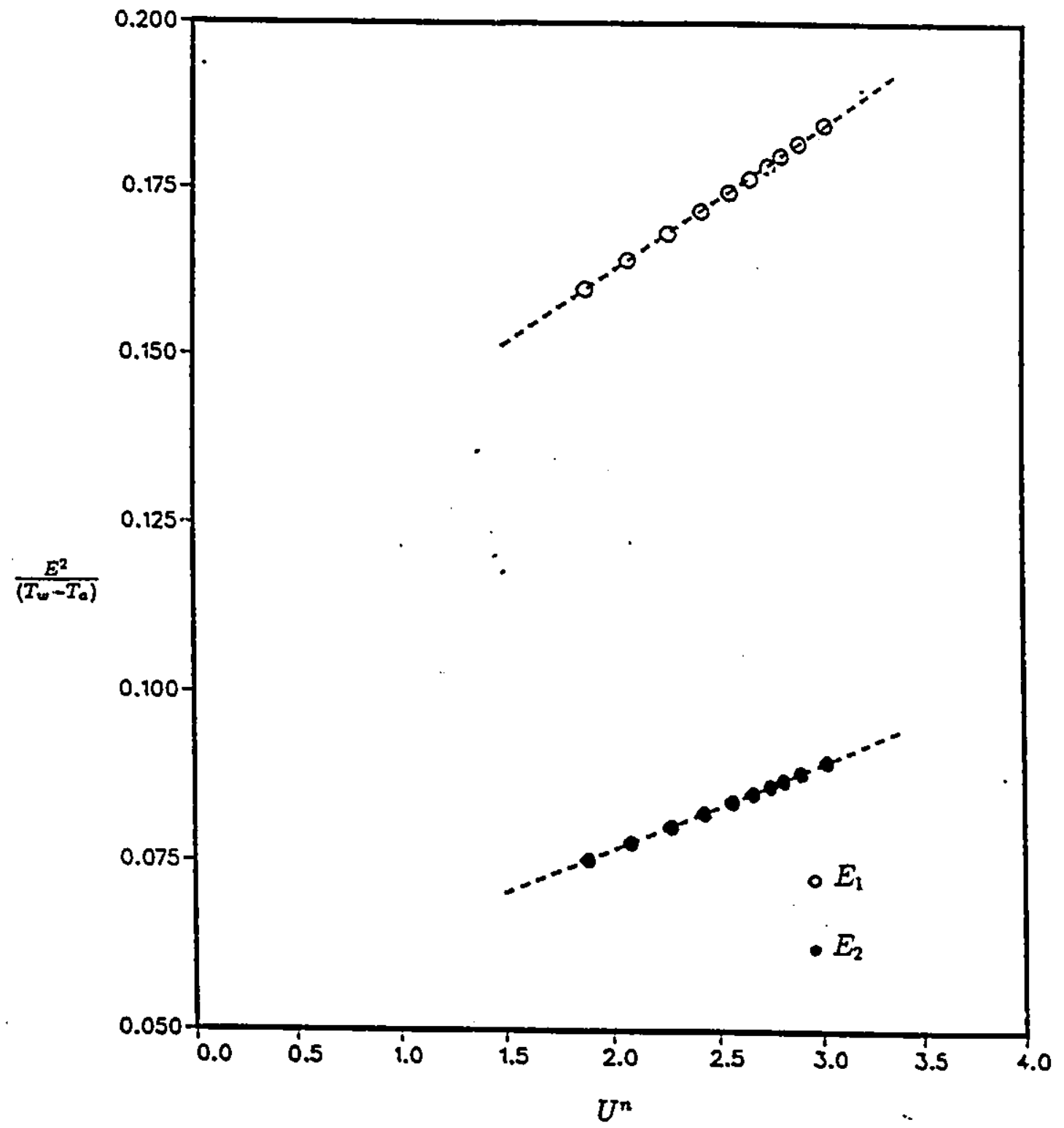

Figure 5.7: Typical calibration curve for hot wires. 


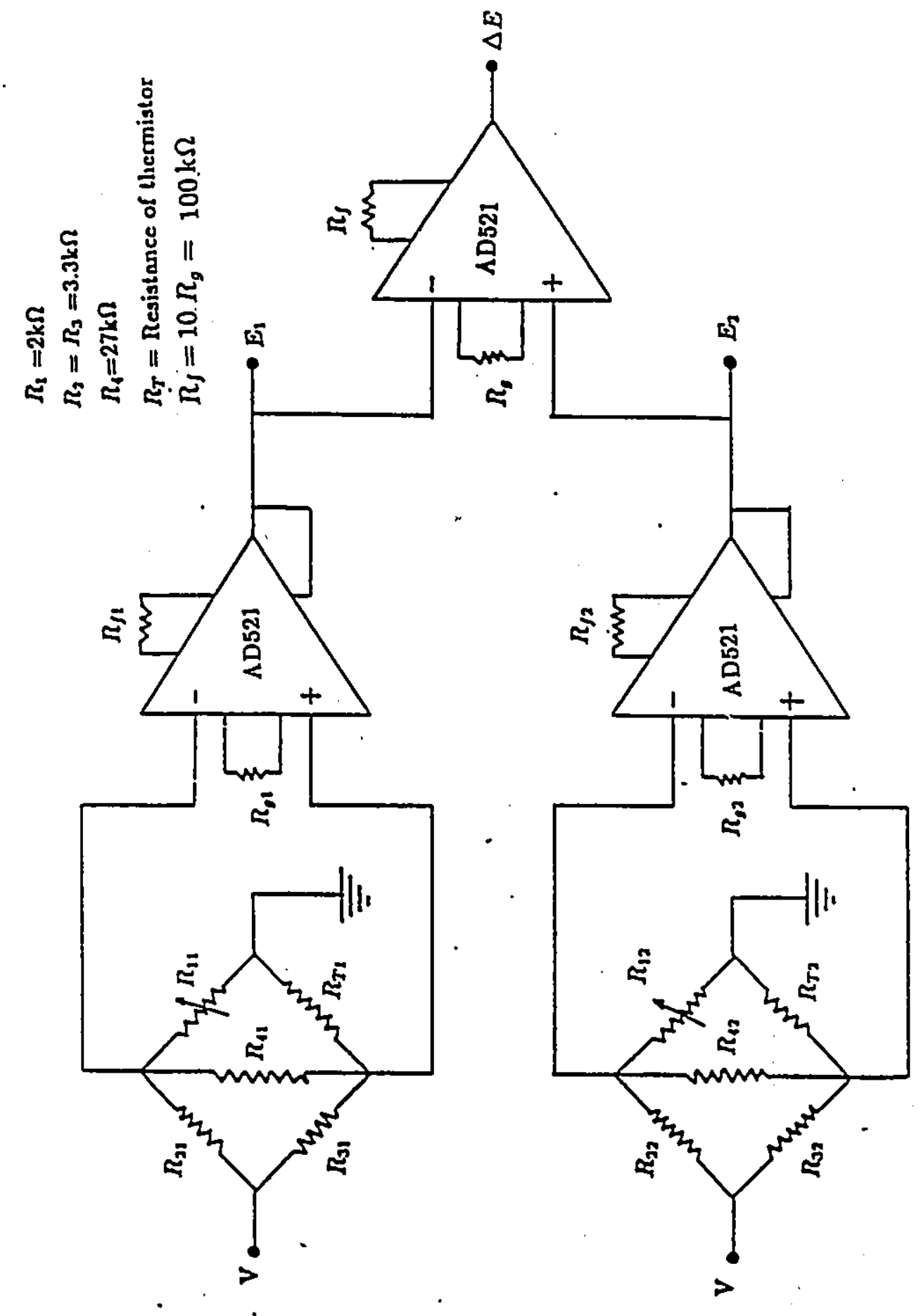

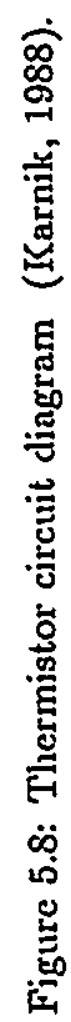



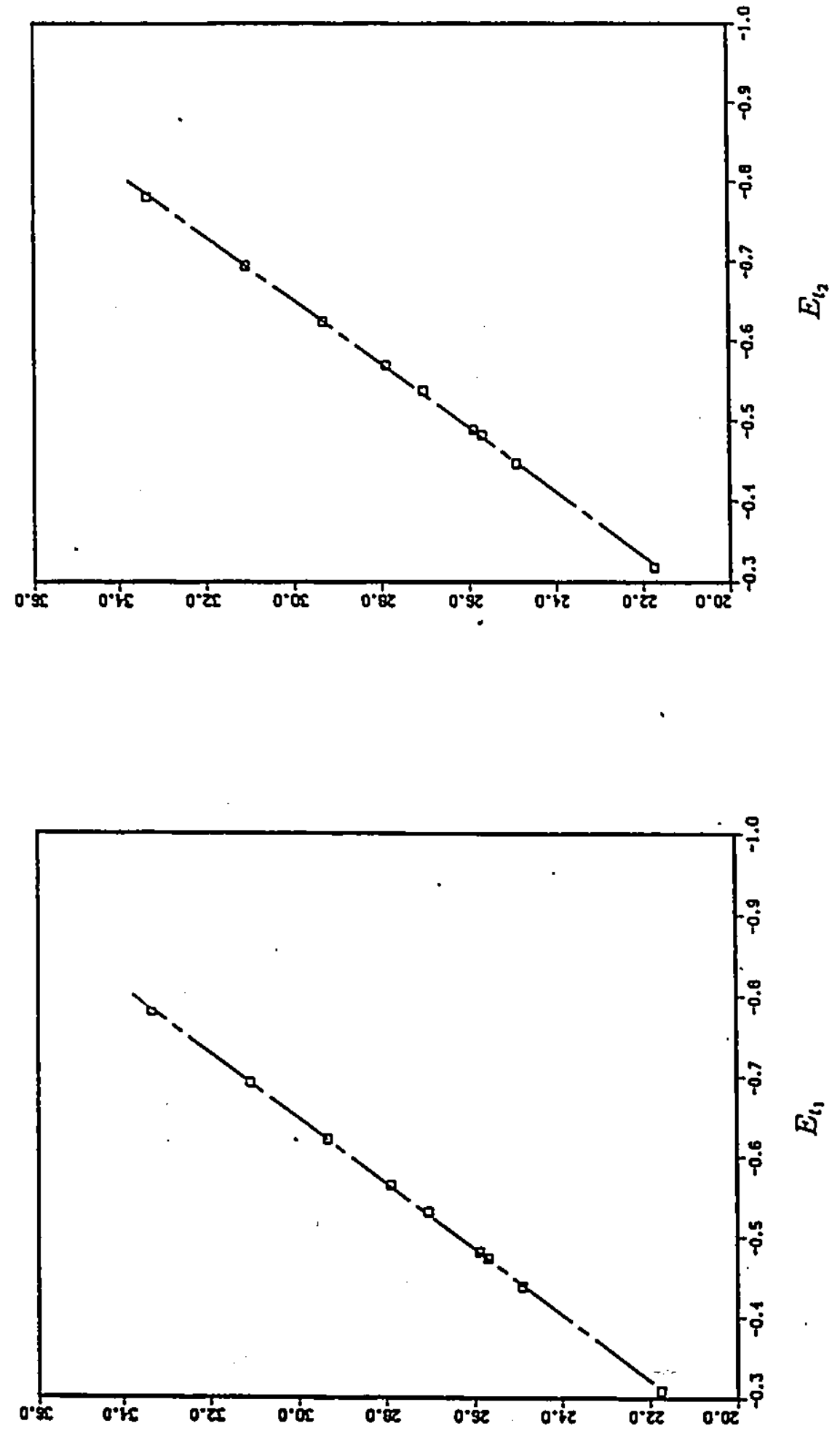

Eั 

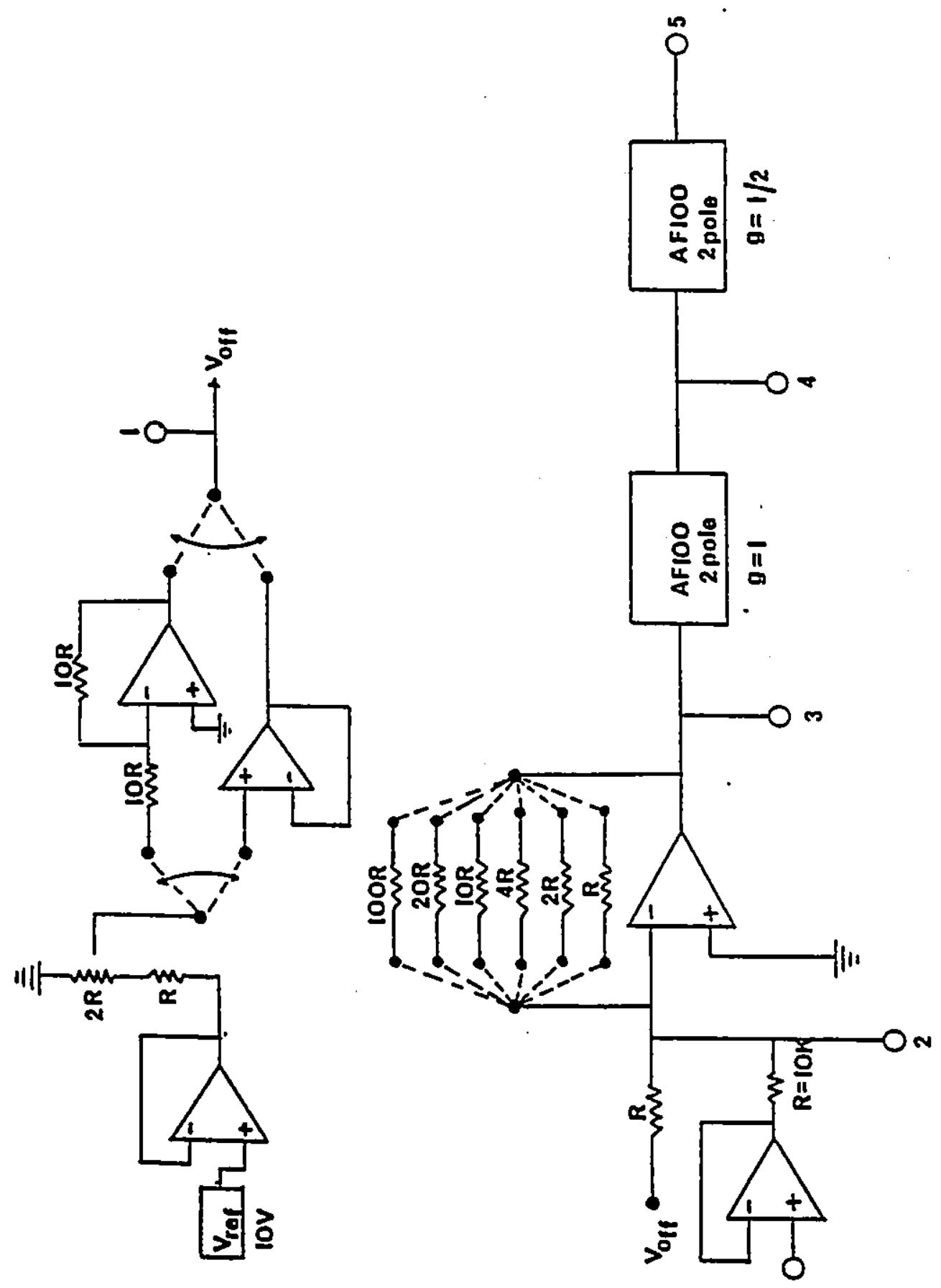

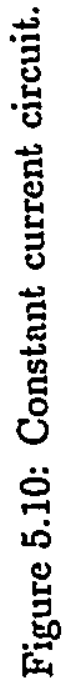




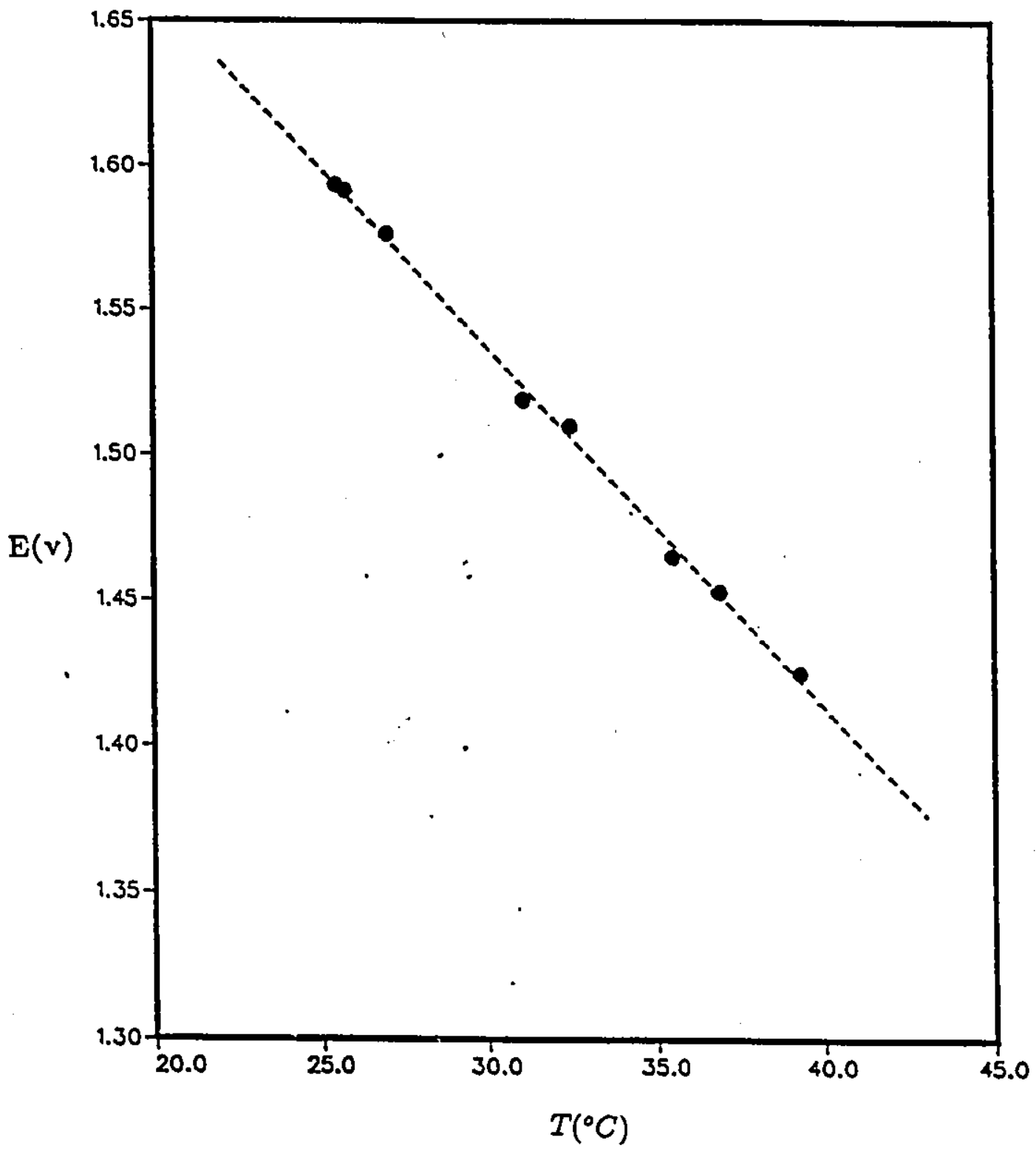

Figure 5.11: Typical calibration curve for cold wire. 

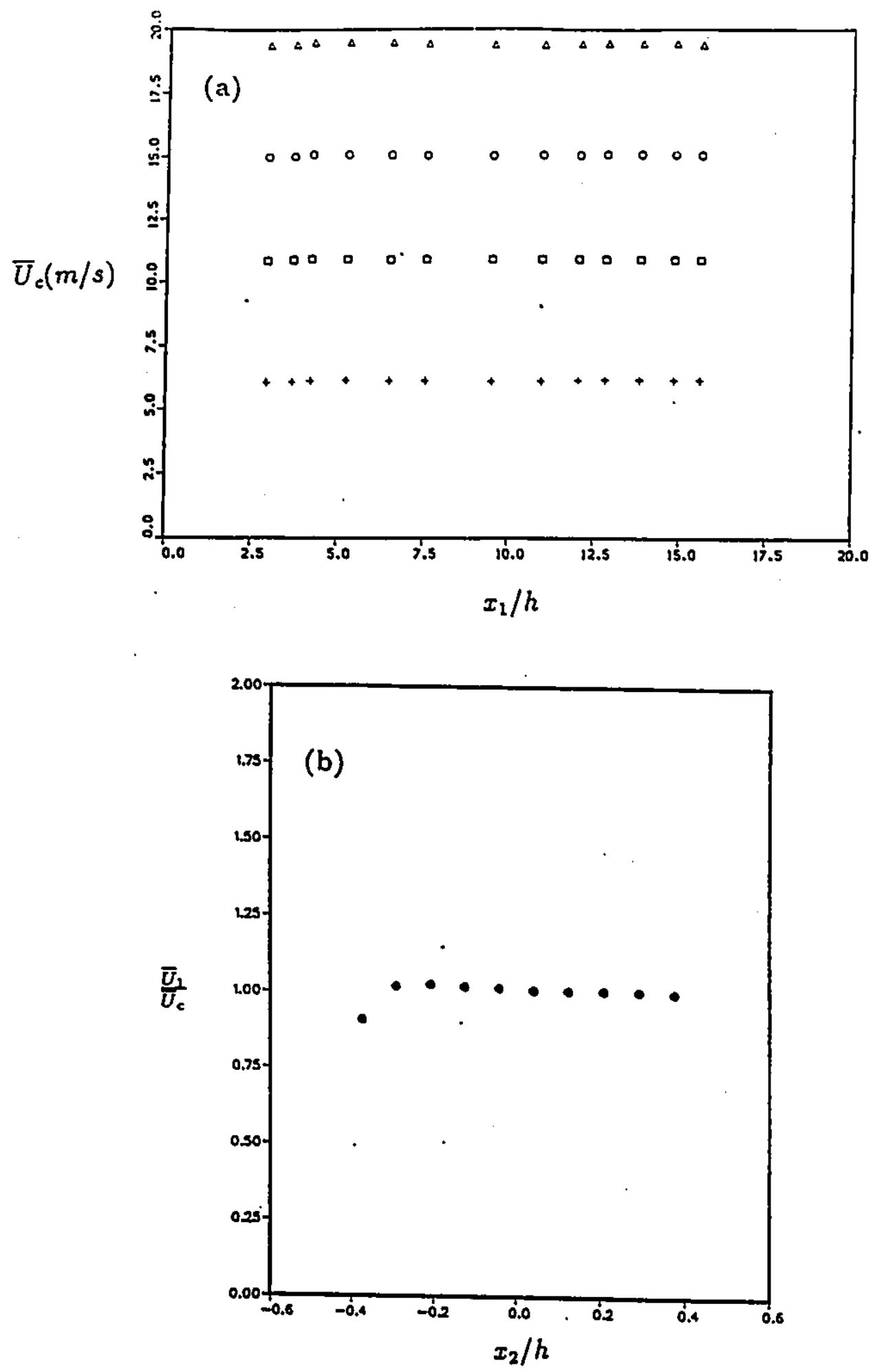

Figure 6.1: Free stream velocity measurements. (a) centerline velocity, (b) transverse profile. 


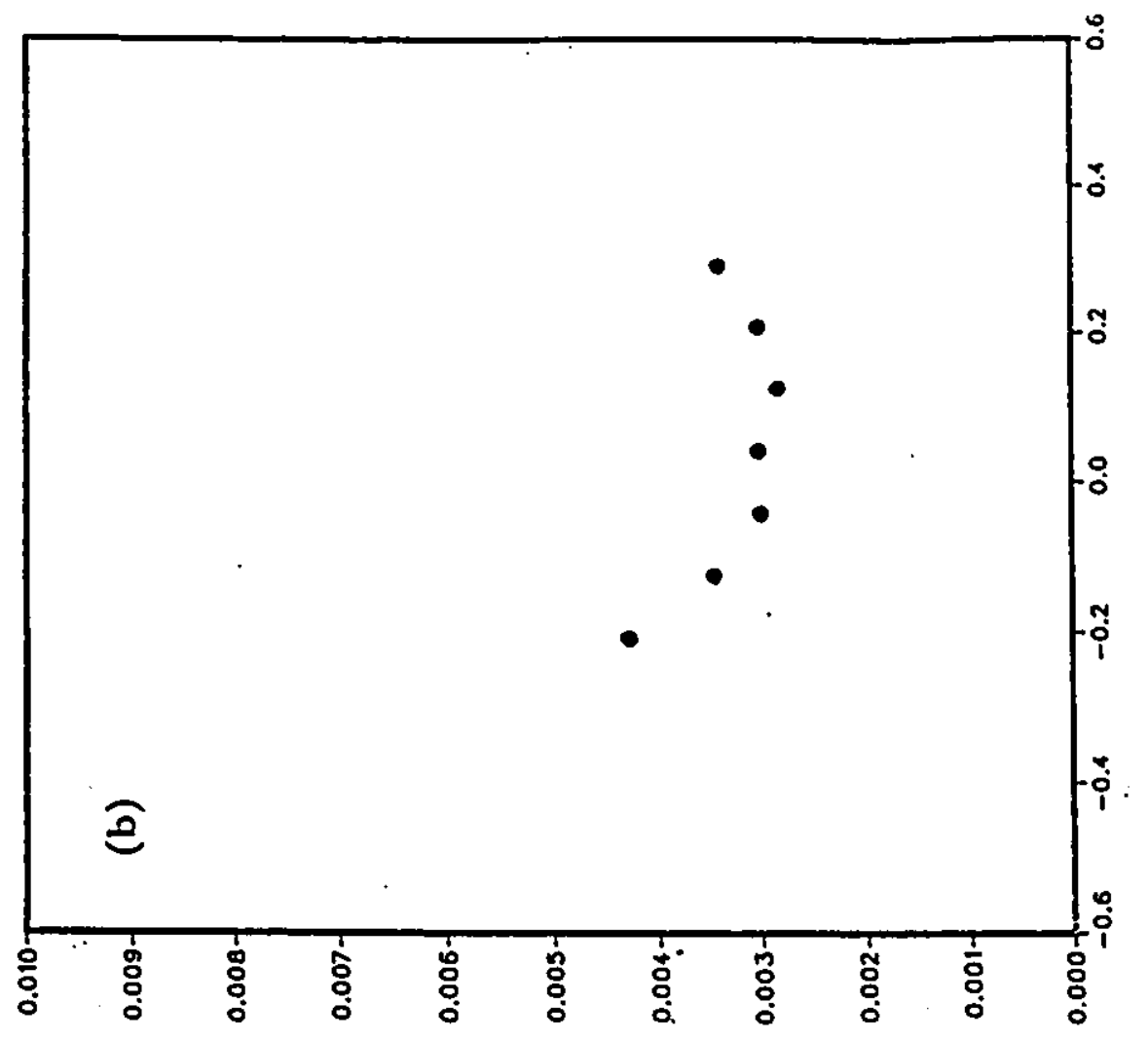

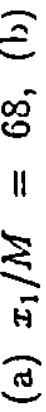

灾

苞

인

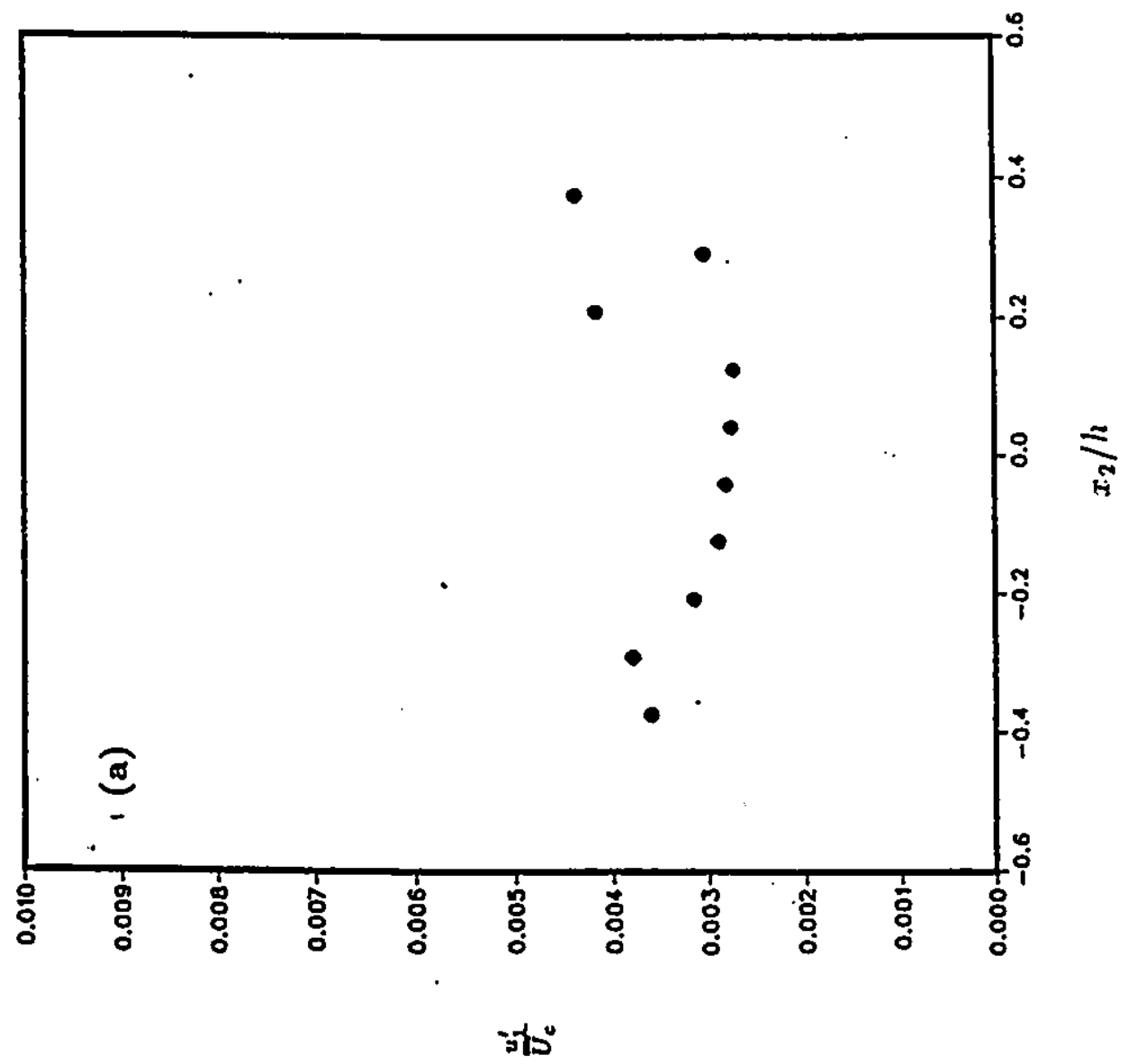

.

总

ن 

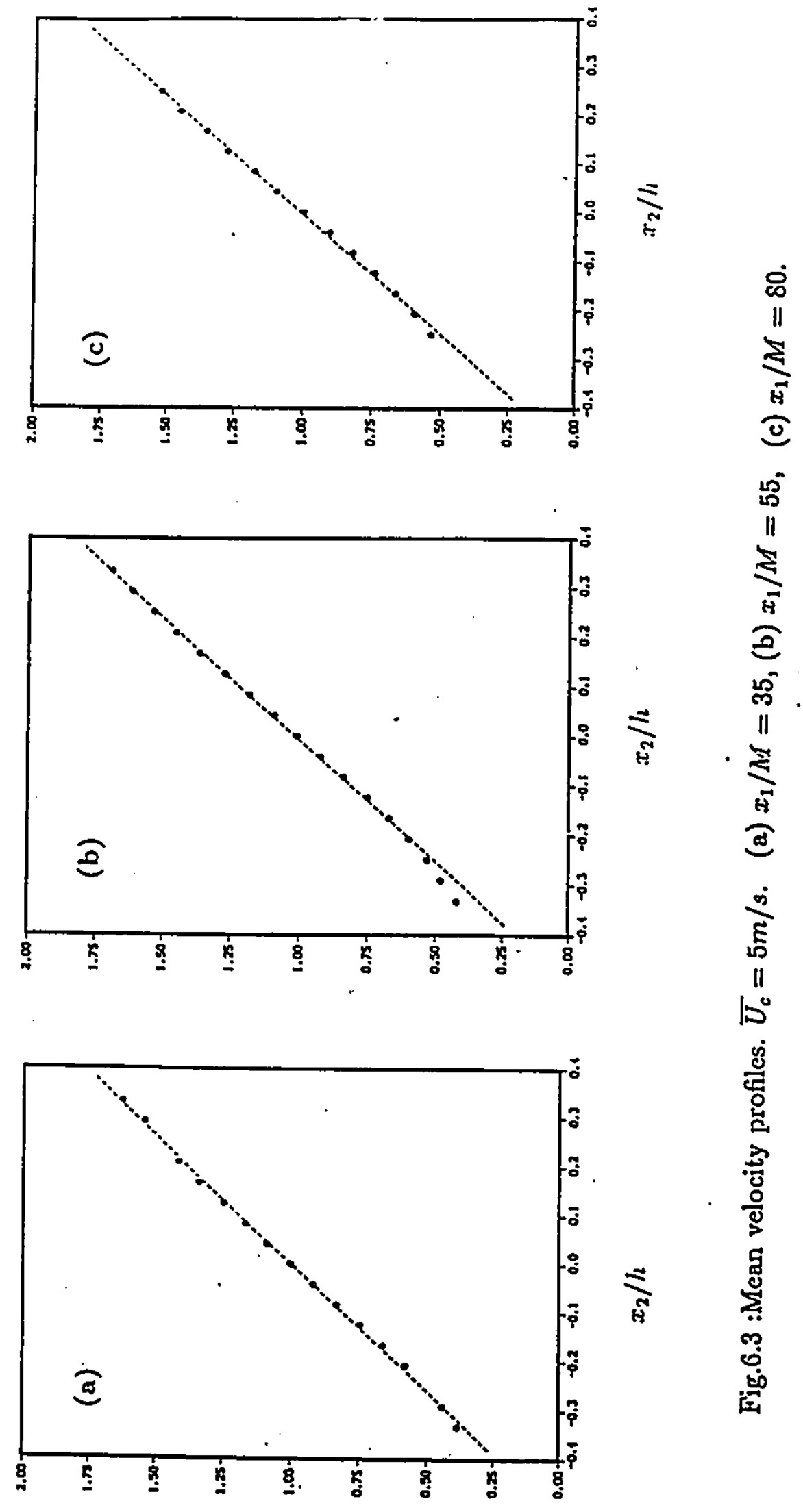

เง

15150 

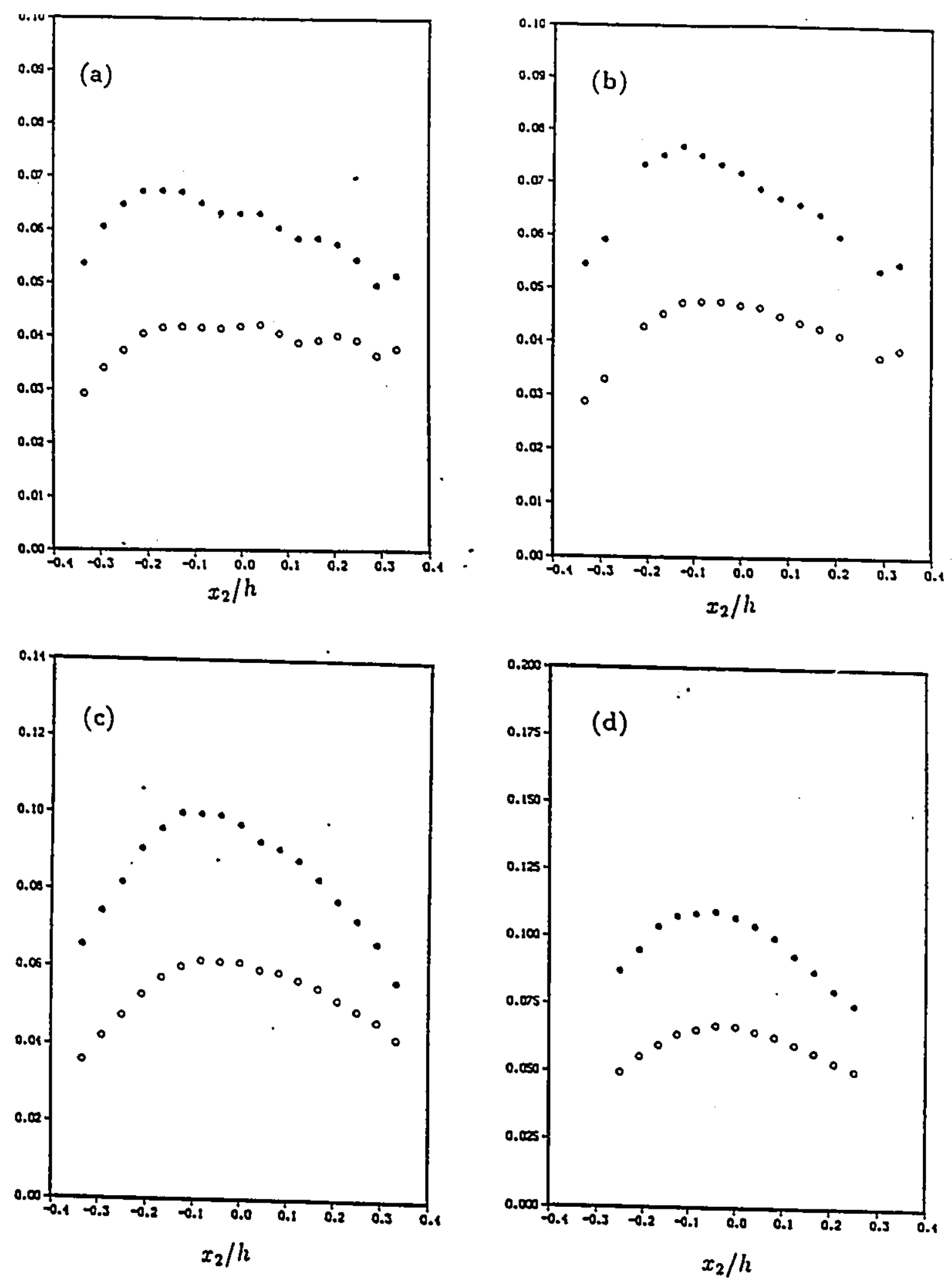

Figure 6.4: Variations of streamivise and transverse turbulent intensity. $x_{1} / M=15$, (b) $x_{1} / M=35$, (c) $x_{1} / M=70$, (d) $x_{1} / M=80$. $\bullet \frac{u_{1}^{\prime}}{U_{c}} \circ \frac{u_{2}^{\prime}}{U_{c}}$ 

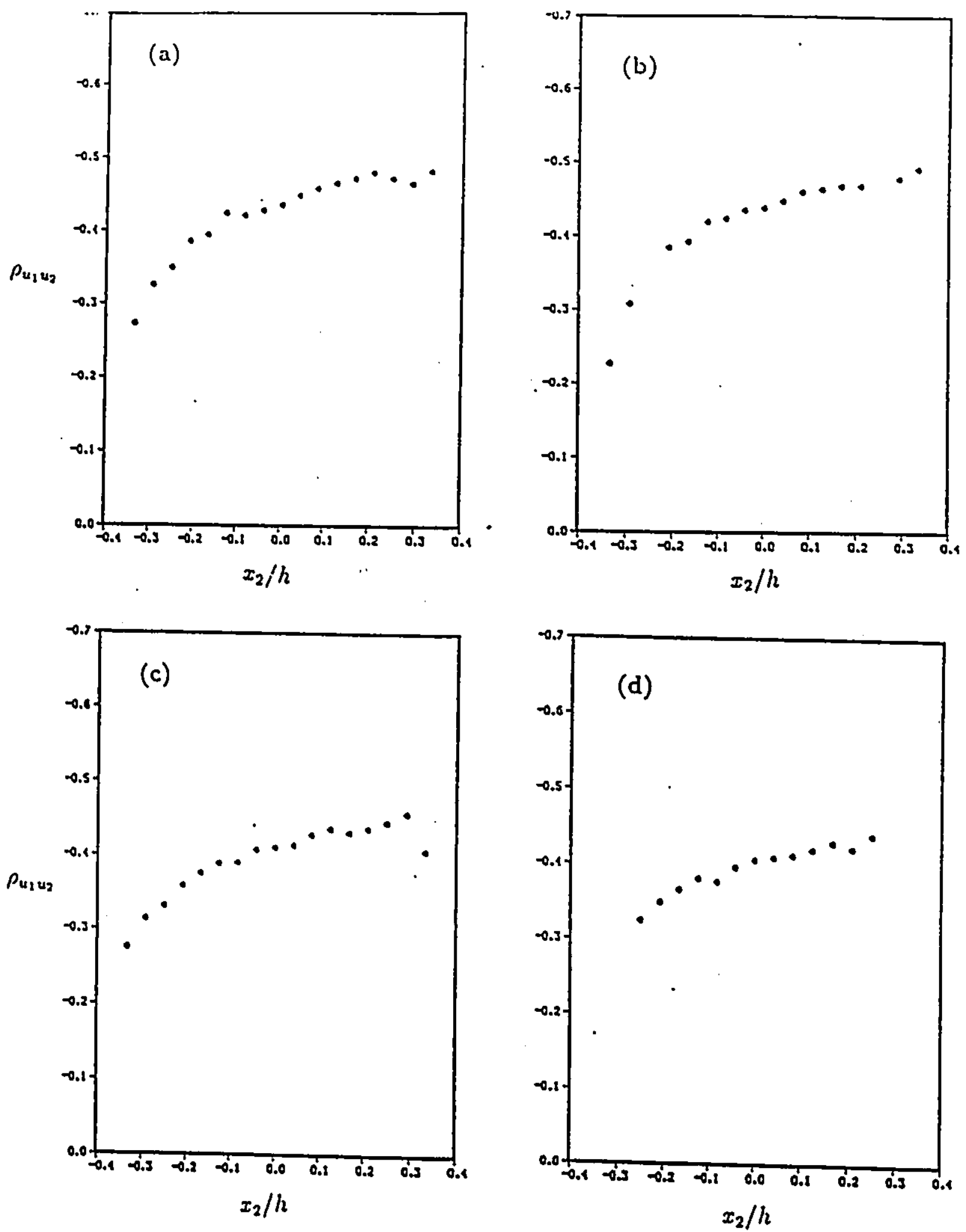

Figure 6.5̄: Shear stress coefficient. (a) $x_{1} / M=15$, (b) $x_{1} / M=35$, (c) $x_{1} / M=70$, (d) $x_{1} / M I=S 0$. 


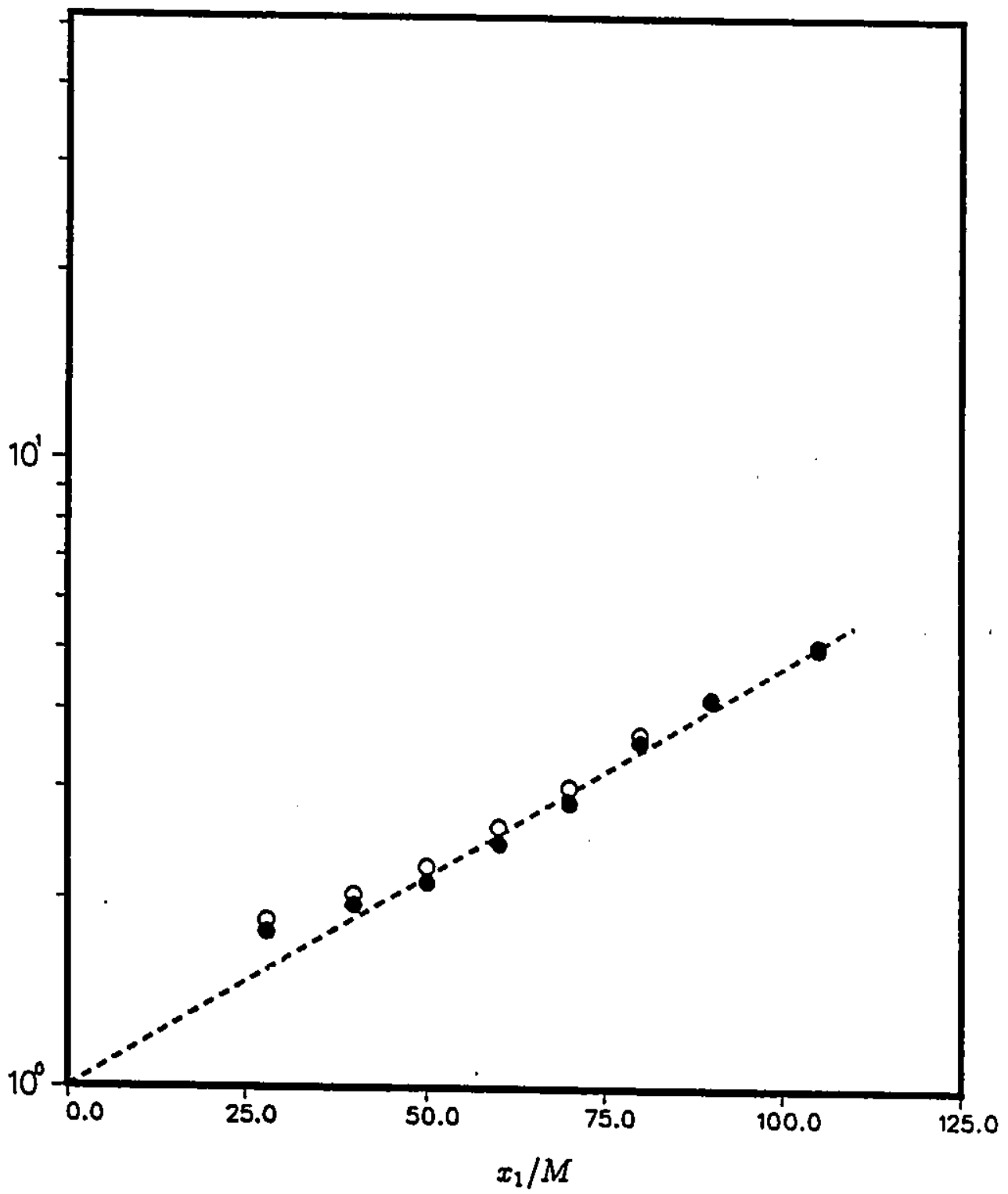

Figure 6.6: Streamwise growth of turbulent stresses; $-\overline{u_{1}^{2}} / \overline{u_{1,0}^{2}} \circ \overline{u_{2}^{2}} / \overline{u_{2,0}^{2}}$ ----Tavoularis and Karnik (1990). 

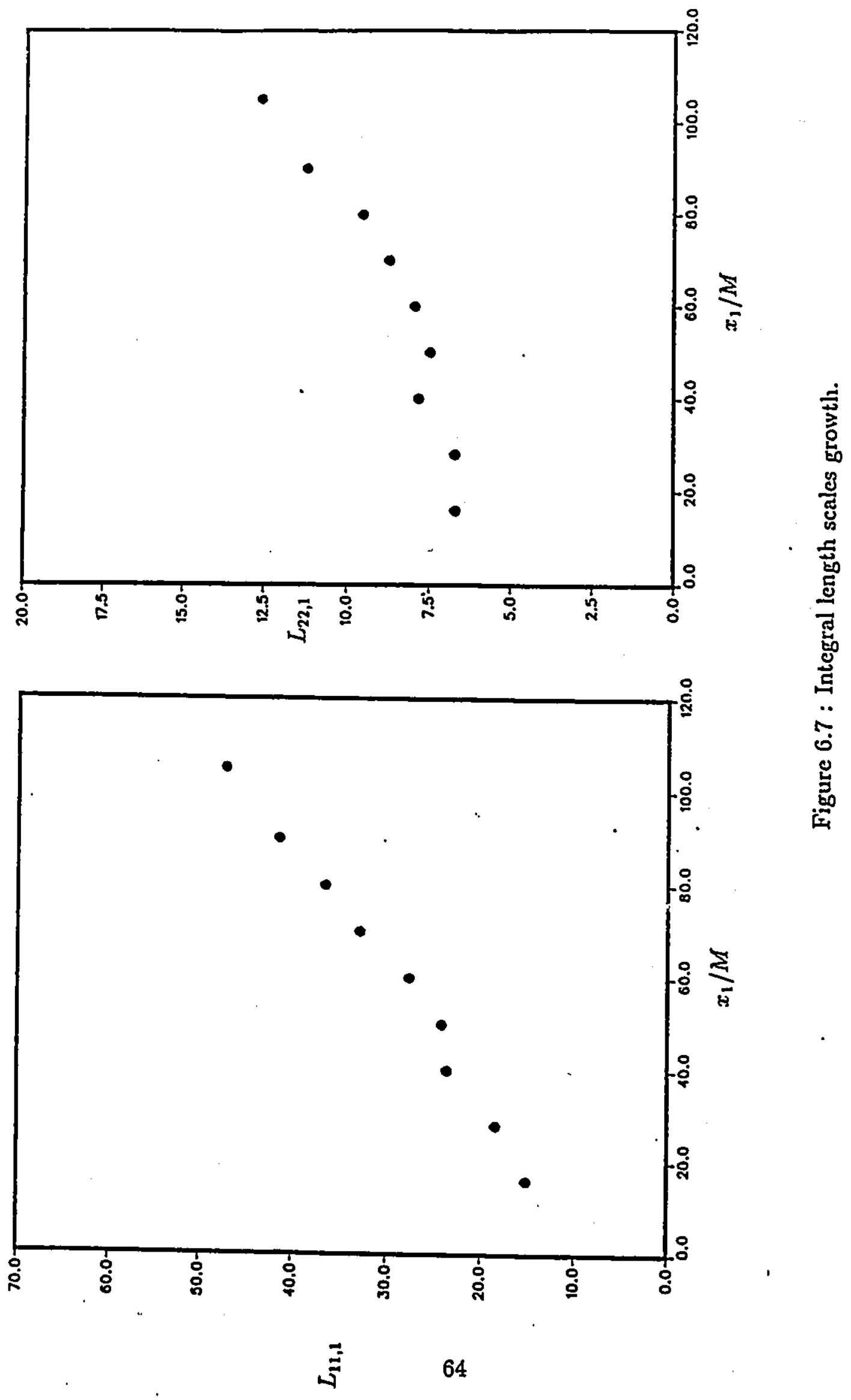


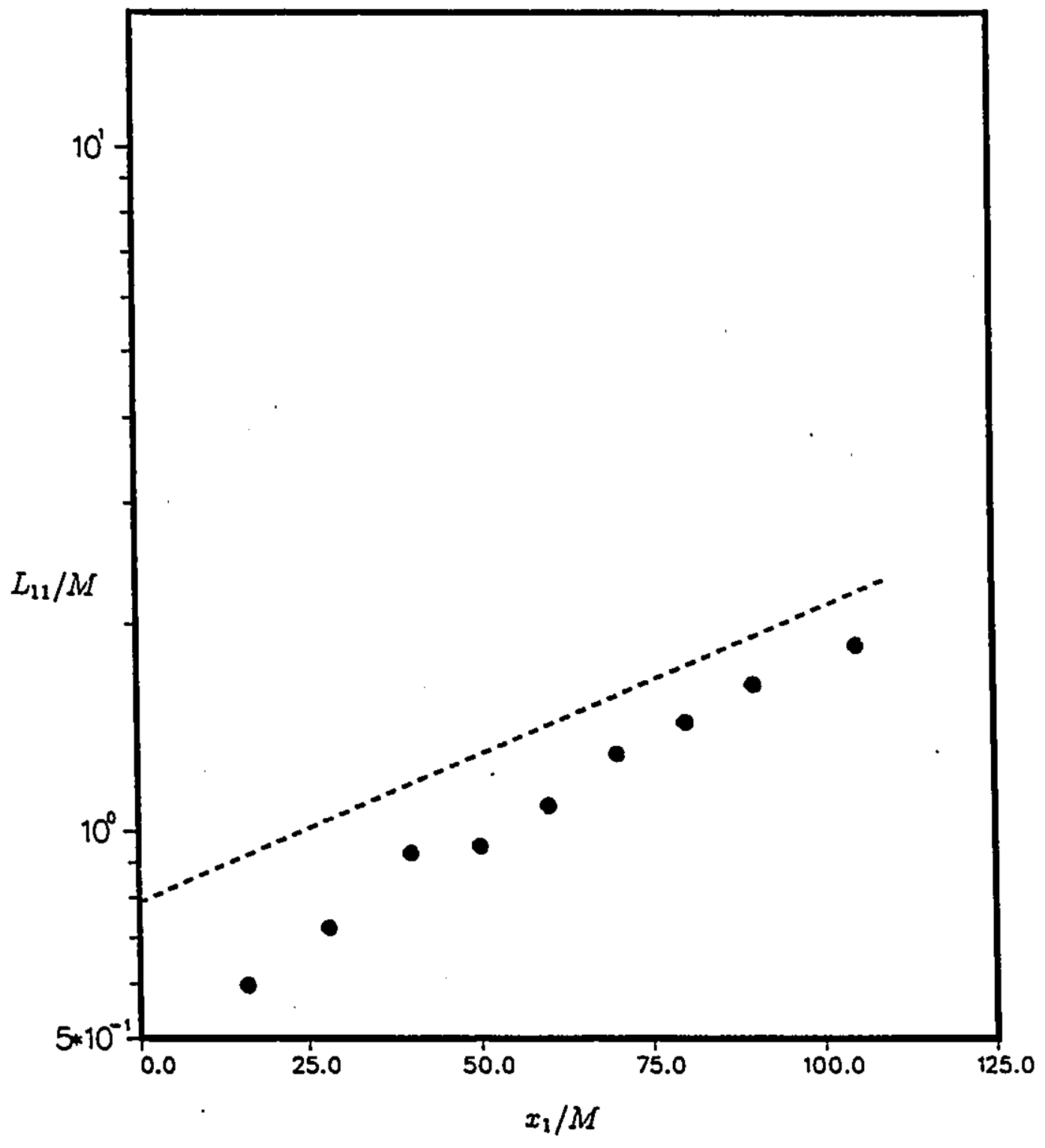

Figure 6.8: sreamwise growth of $L_{11}$; • present experiment -..- from Tavoularis and Karnik (1990). 

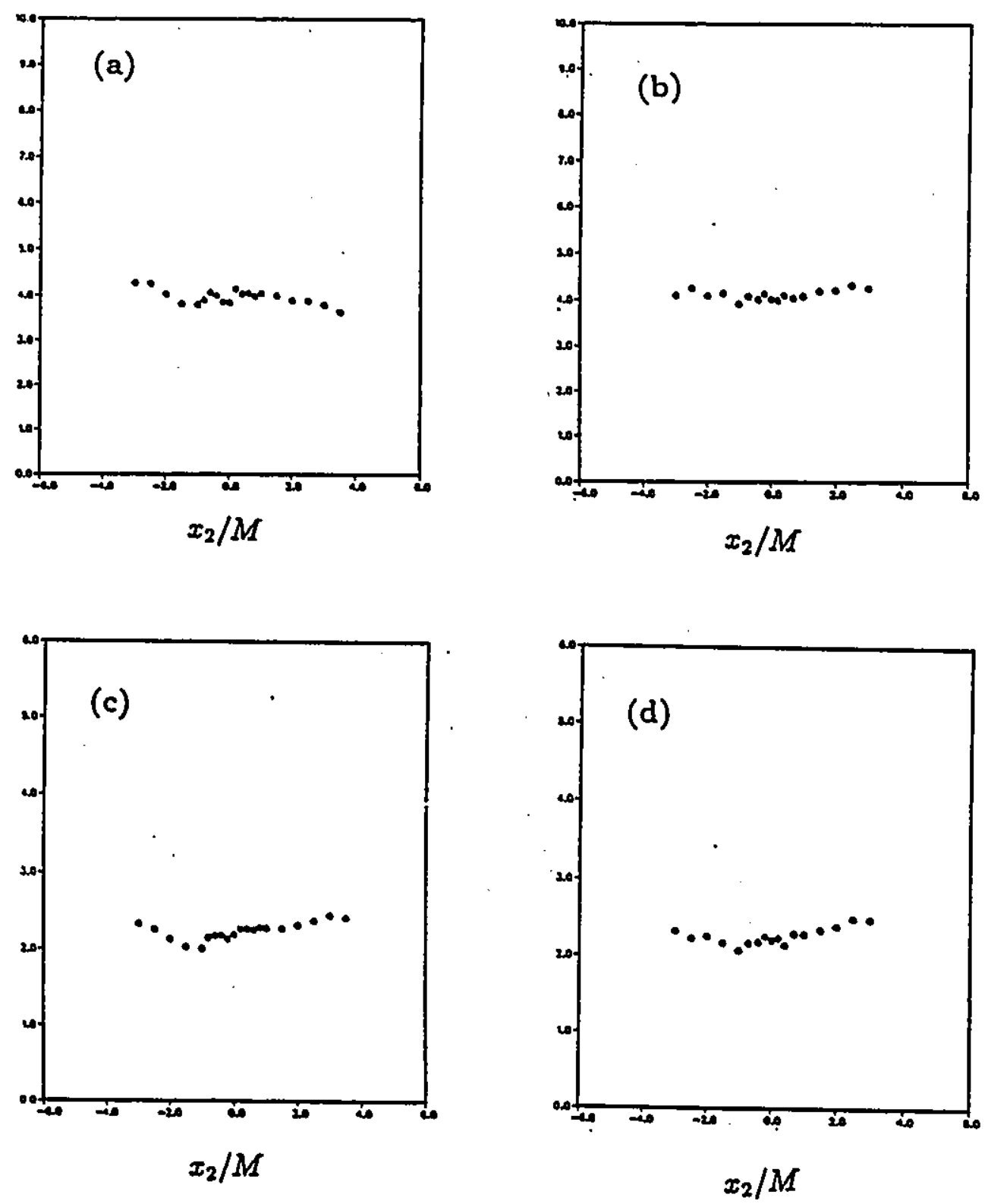

Figure 6.9: Transverse profiles of Taylor microscales. (a) $\lambda_{11}$ at $x_{1} / M=40$, (b) $\lambda_{11}$ at $x_{1} / M=60$, (c) $\lambda_{22}$ at $x_{1} / M=40$, (d) $\lambda_{22}$ at $x_{1} / M=60$. 


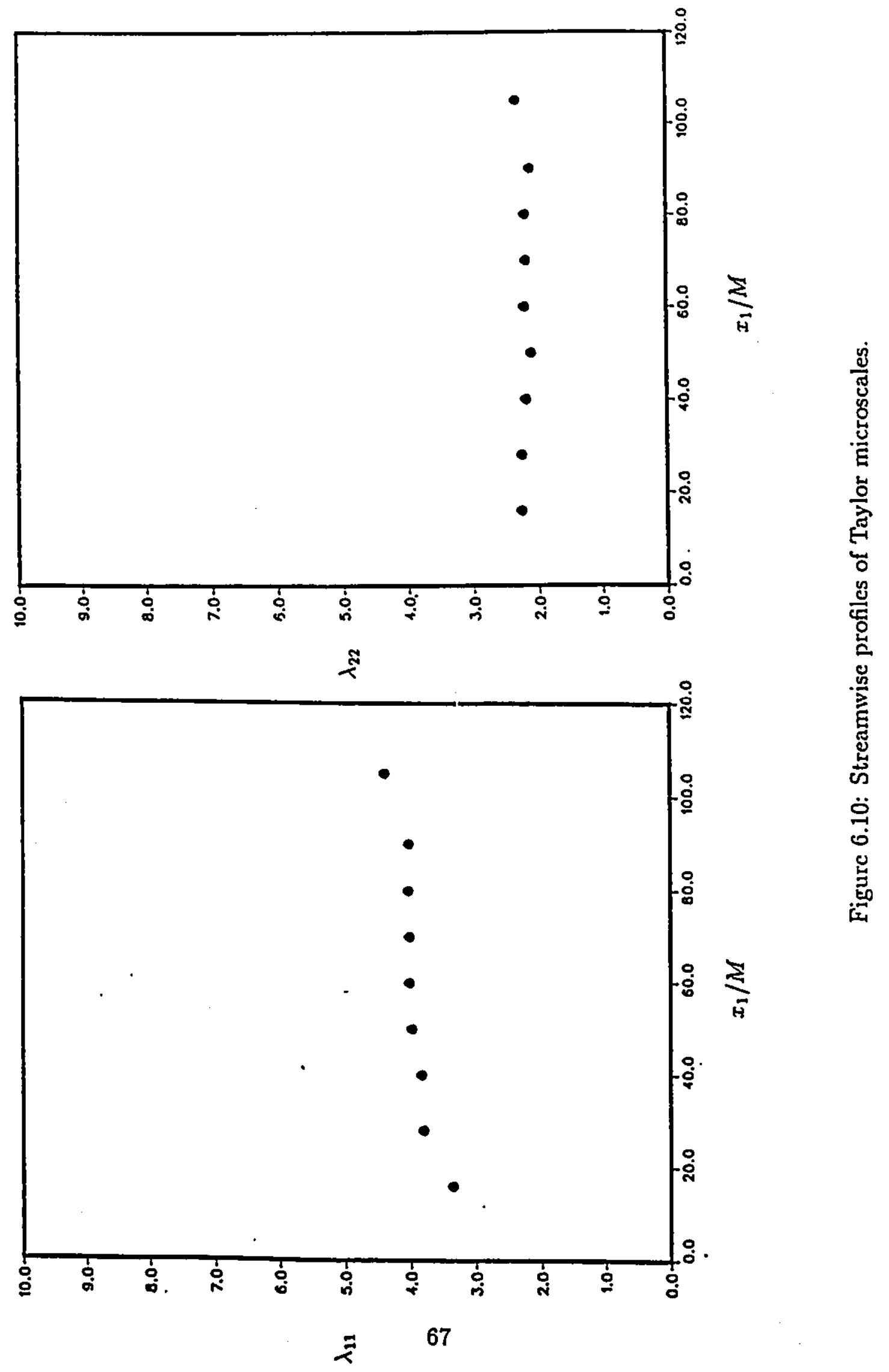




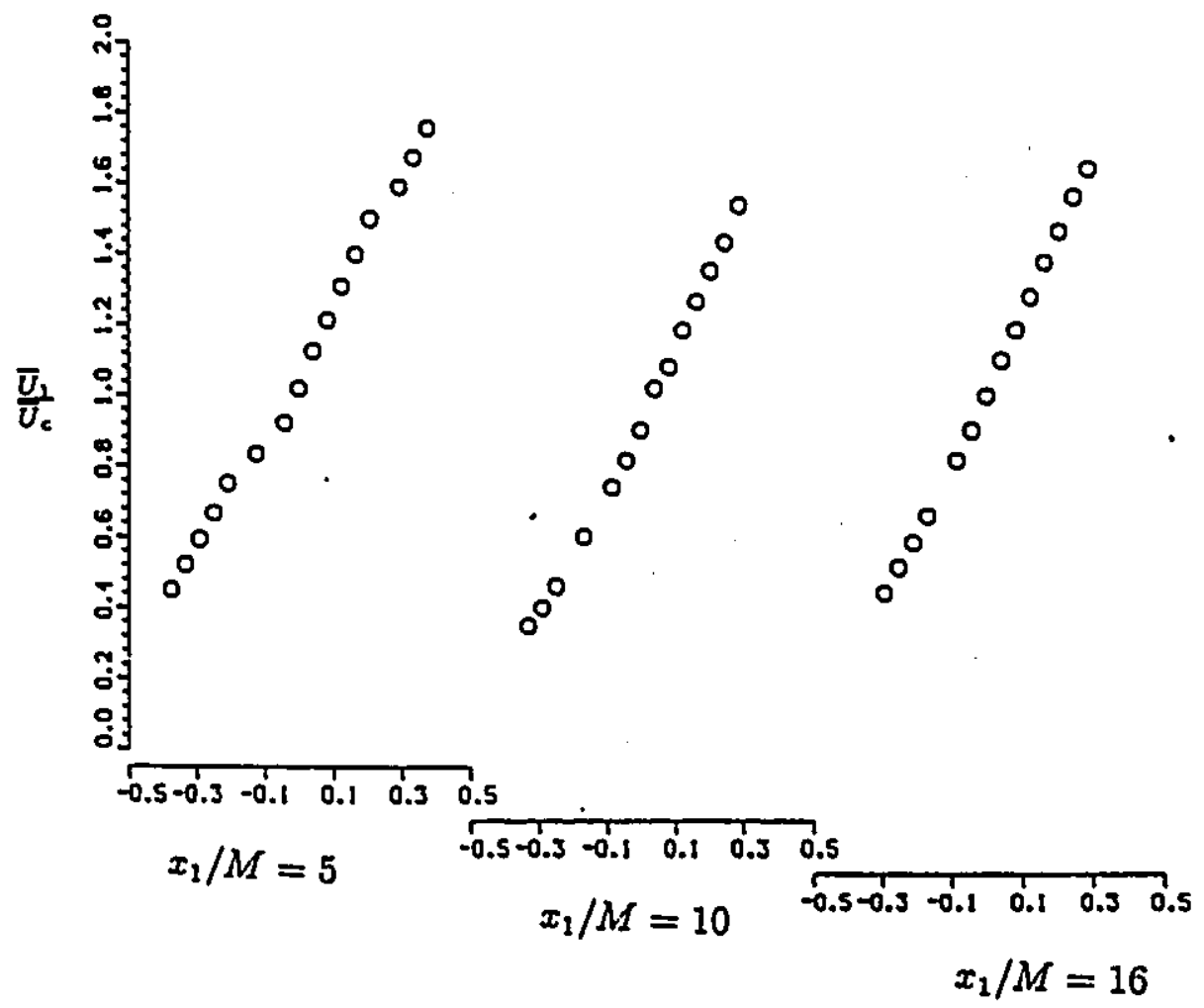

Figure 6.11: Velocity profiles with the presence of the heating ribbons. 


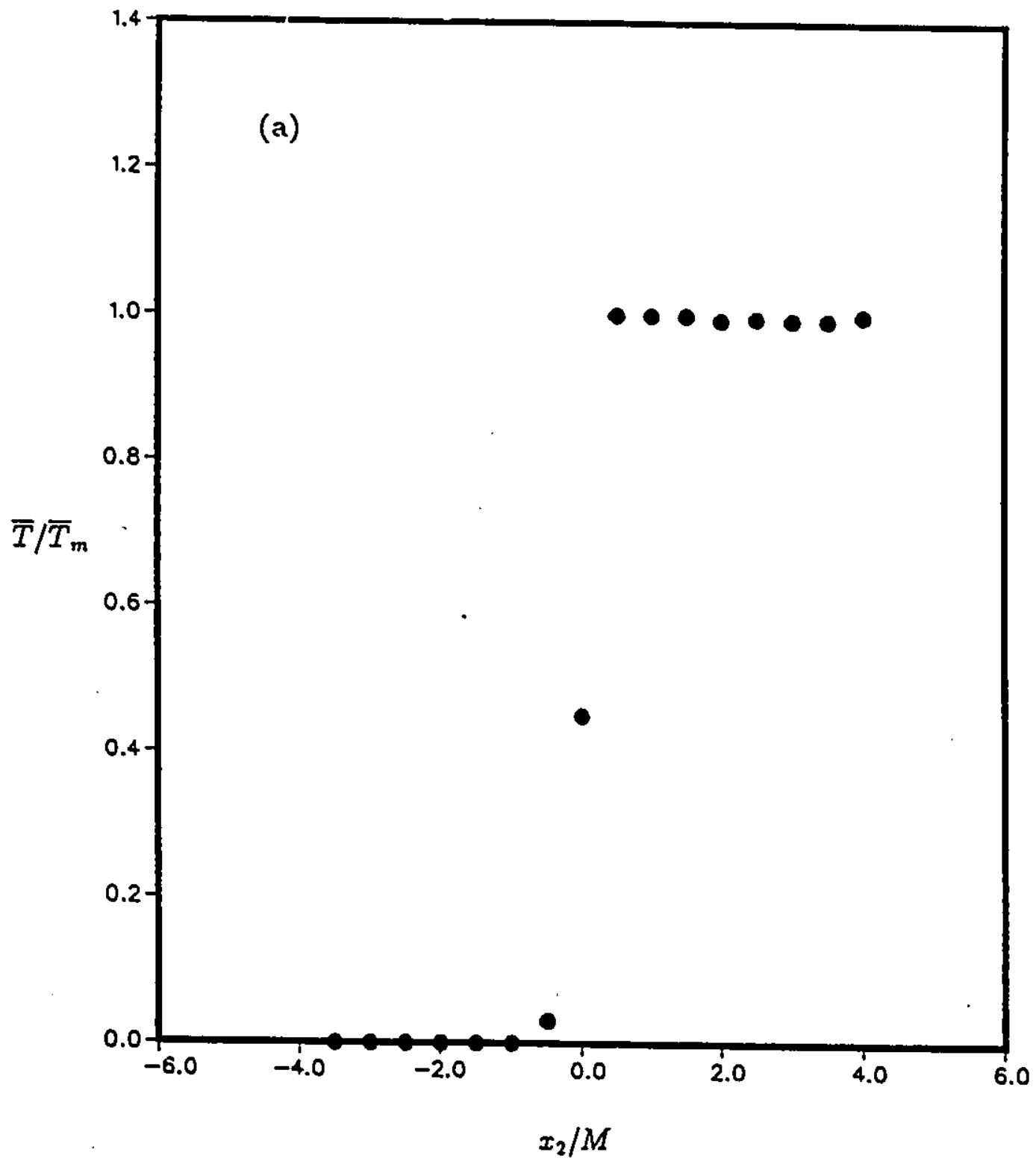

Figure 6.12: Transverse profiles of the mean temperature rise. (a) $x_{1} / M=16$ (b) $x_{1} / M=28$ (c) $x_{1} / M=40$ (d) $x_{1} / M=50$ (e) $x_{1} / M=60$ (f) $x_{1} / M=70(\mathrm{~g})$ $x_{1} / M=80$ (h) $x_{1} / M=90$ (i) $x_{1} / M=105$. 

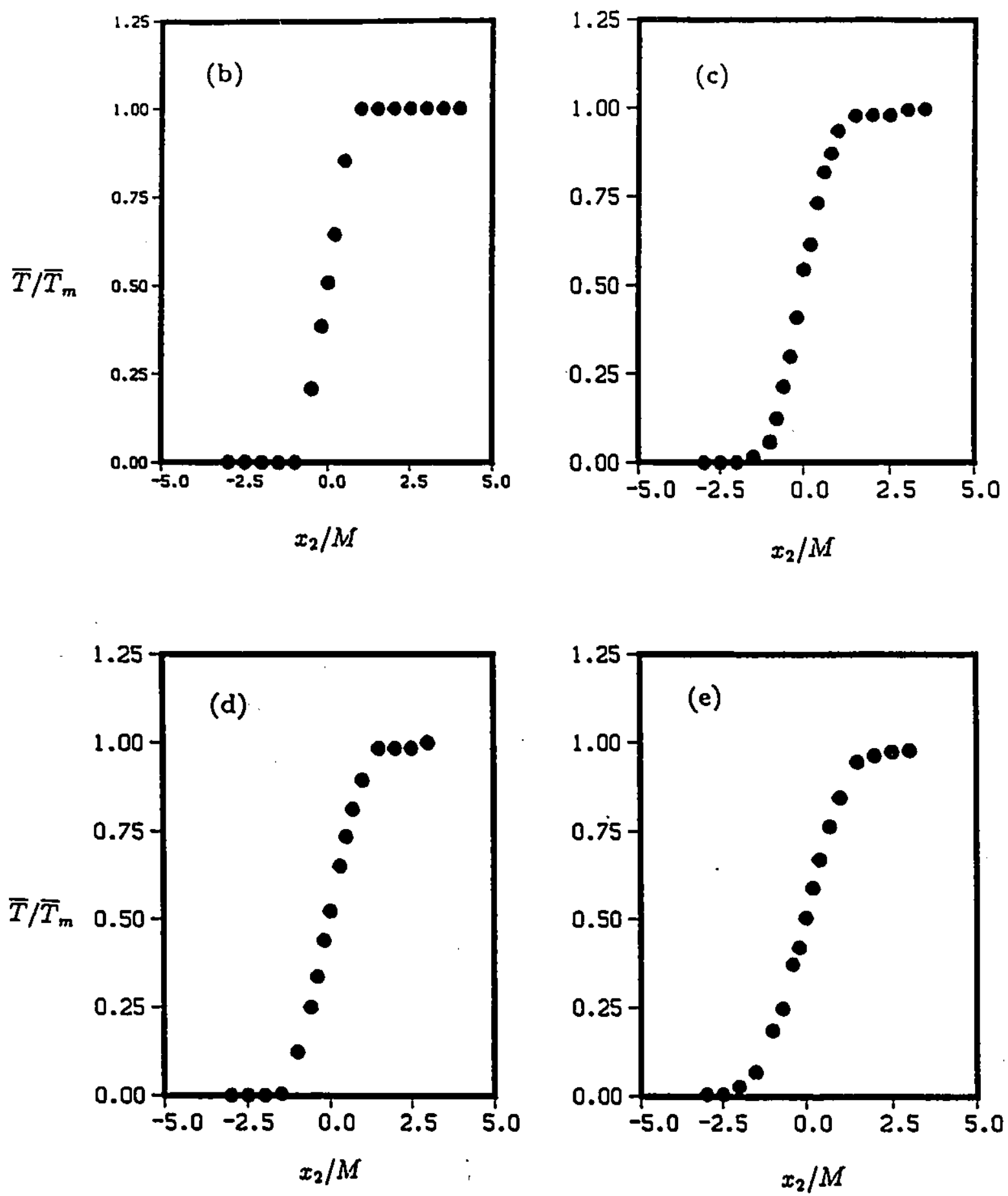

Figure 6.12. cont'd. 

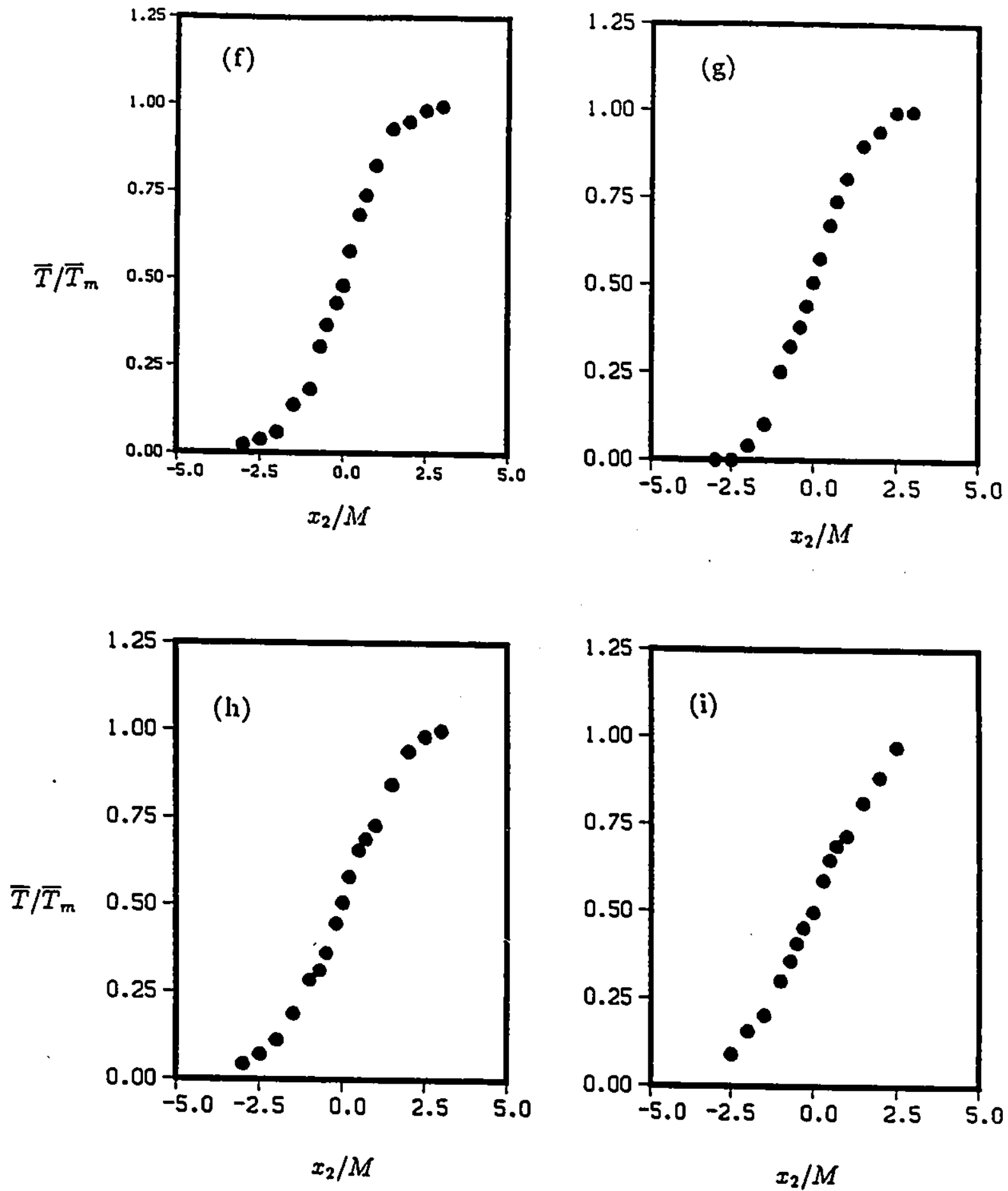

Figure 0.12. cont'd. 


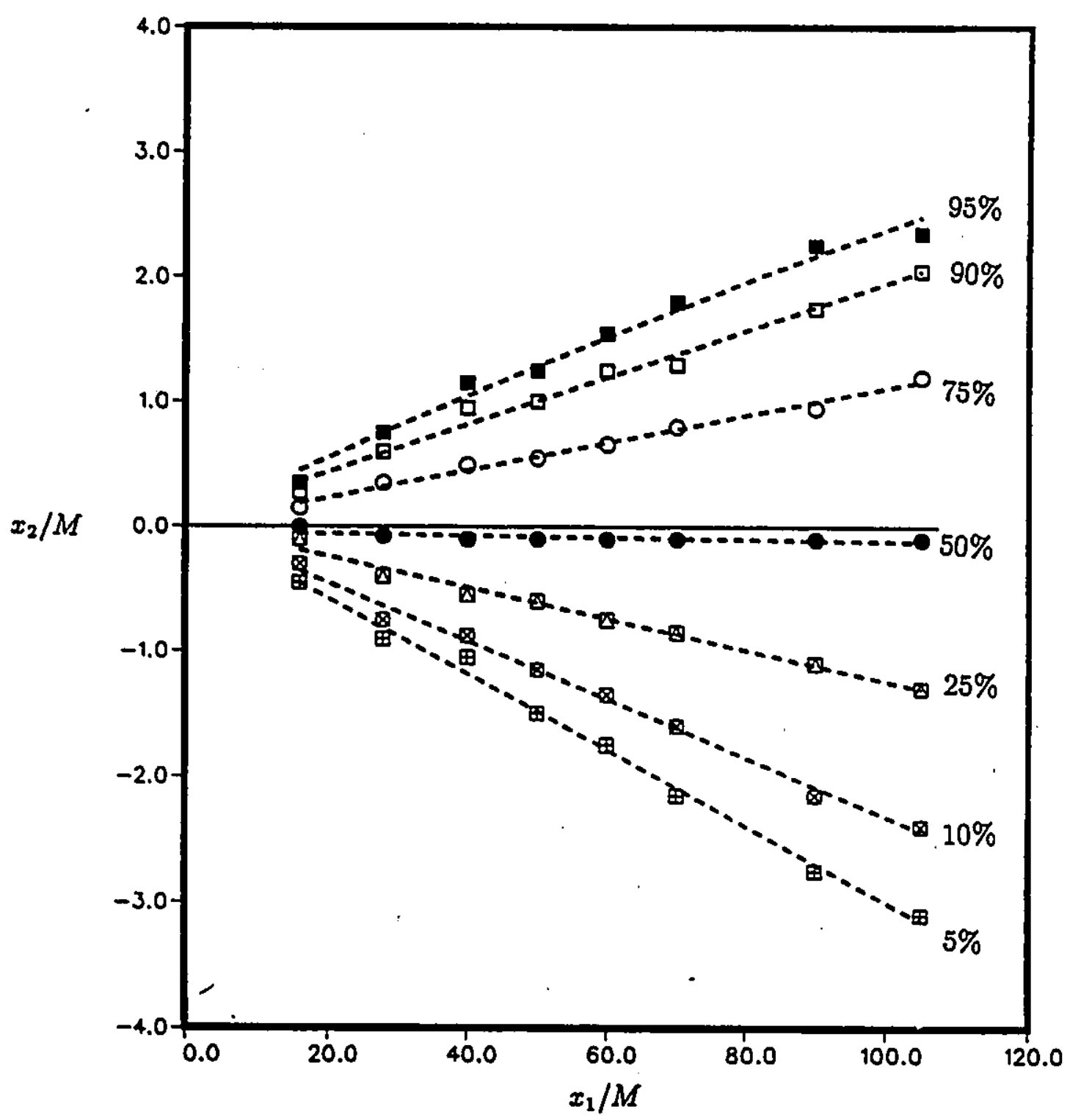

Figure 6.13: Isotherms on the wind tunnel centerplane. 


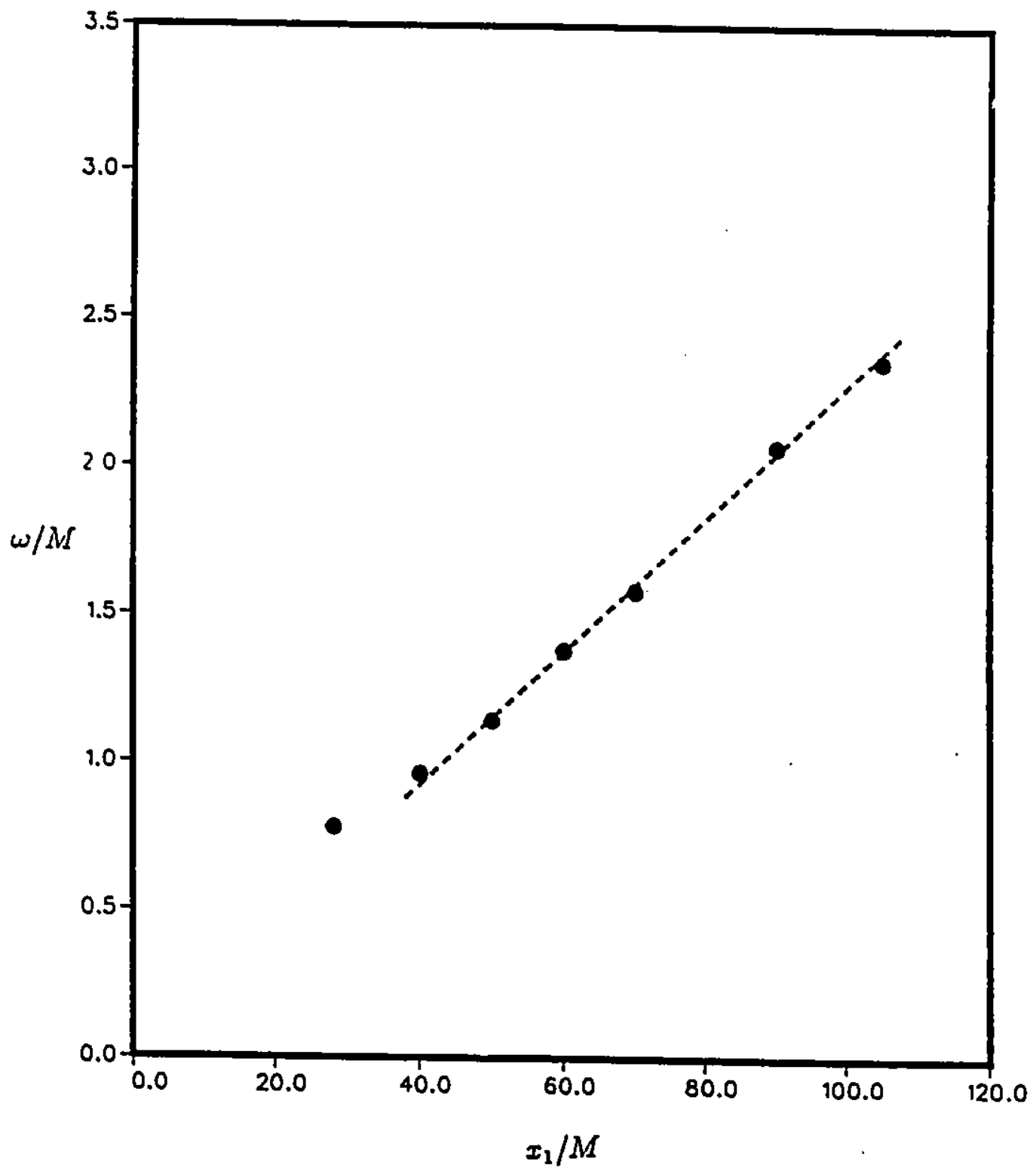

Figure 6.14: Growth of the mixing layer's width. 

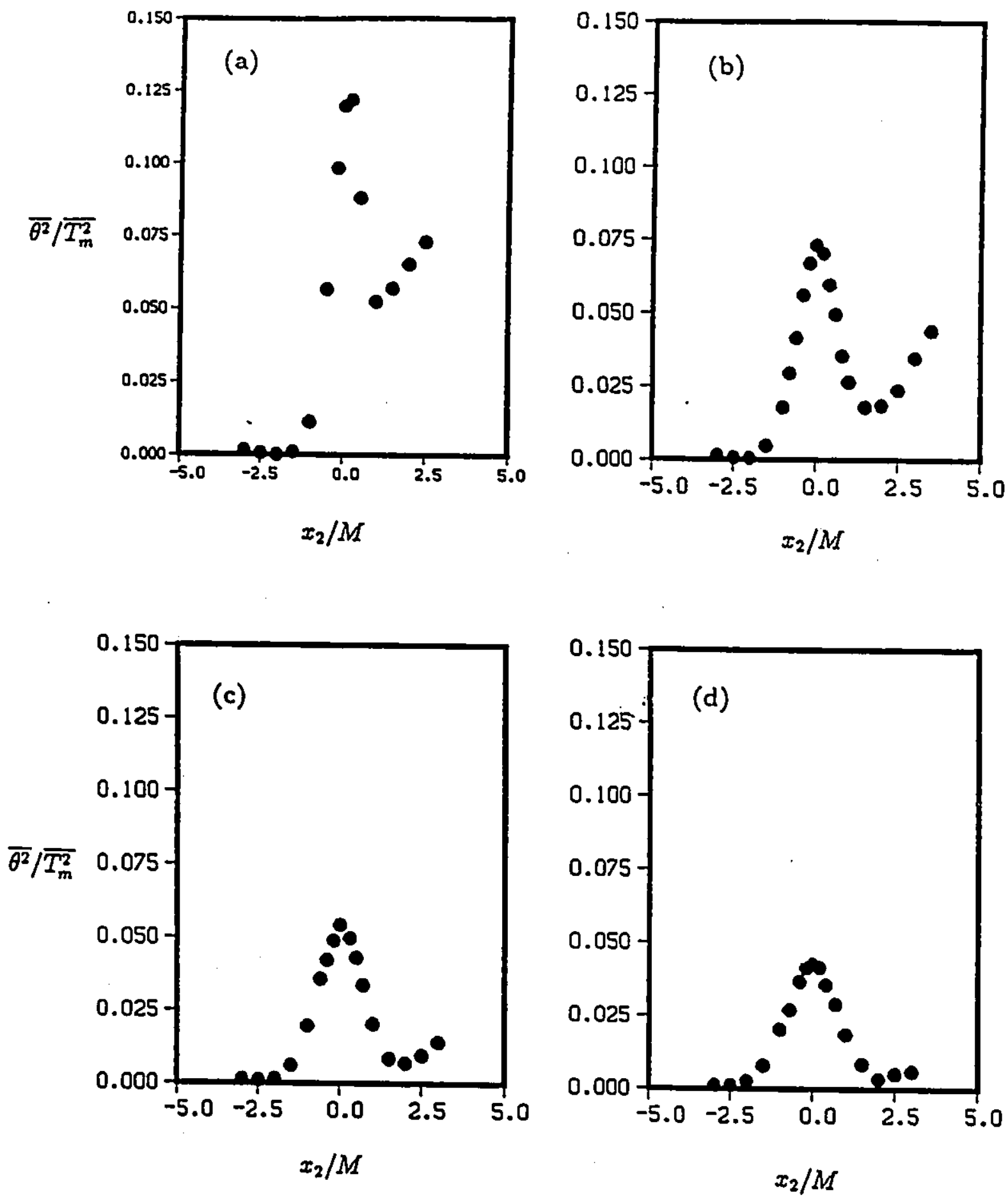

Figure 6.15: Transverse distribution of temperature variance. (a) $x_{1} / M=28$ (b) $x_{1} / M=40$ (c) $x_{1} / M=50$ (d) $x_{1} / M=60$ (e) $x_{1} / M=70$ (f) $x_{1} / M=80(\mathrm{~g})$
$x_{1} / M=90(\mathrm{~h}) x_{1} / M=105$. 

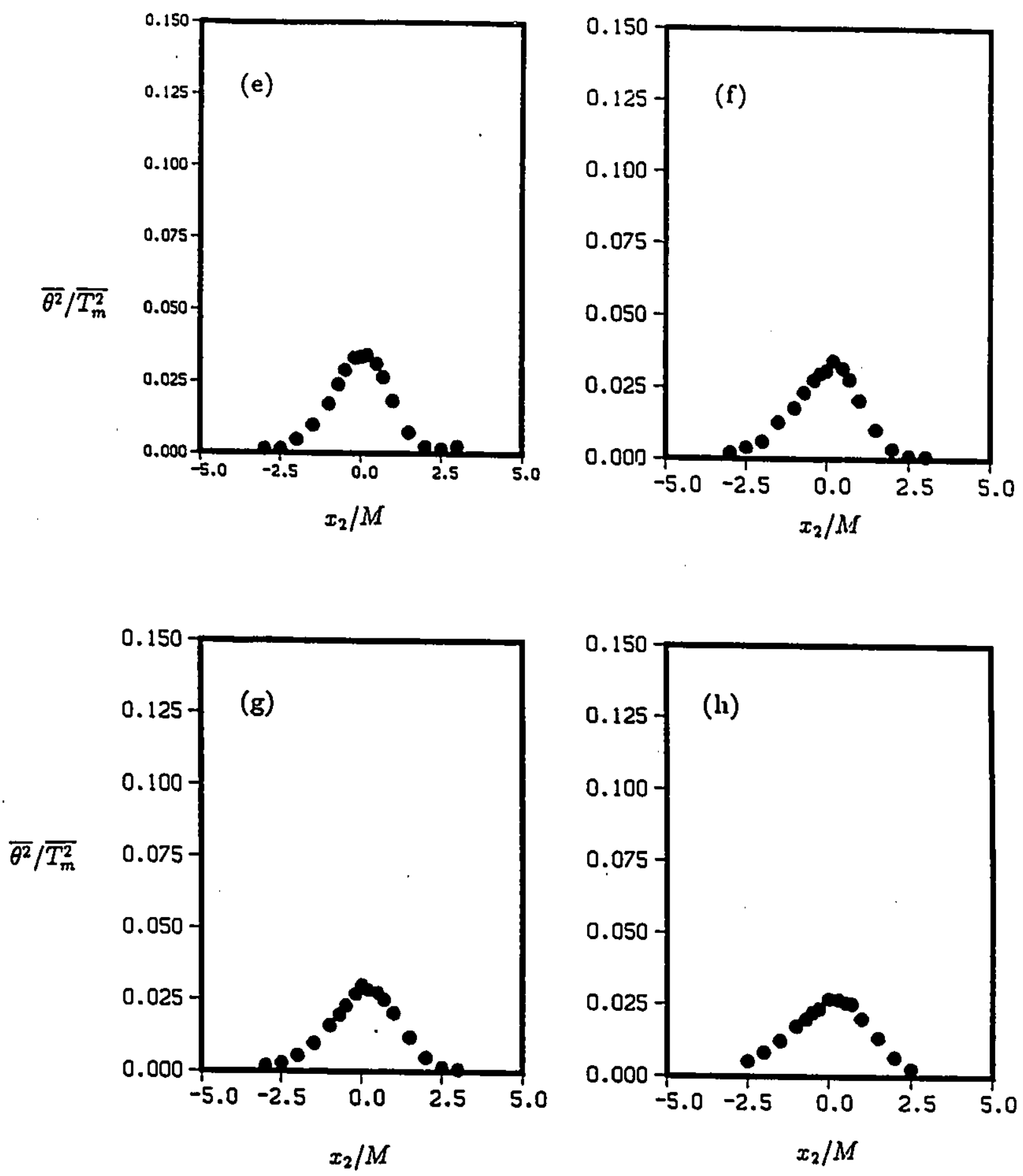

Figure 6.15. cont'd. 


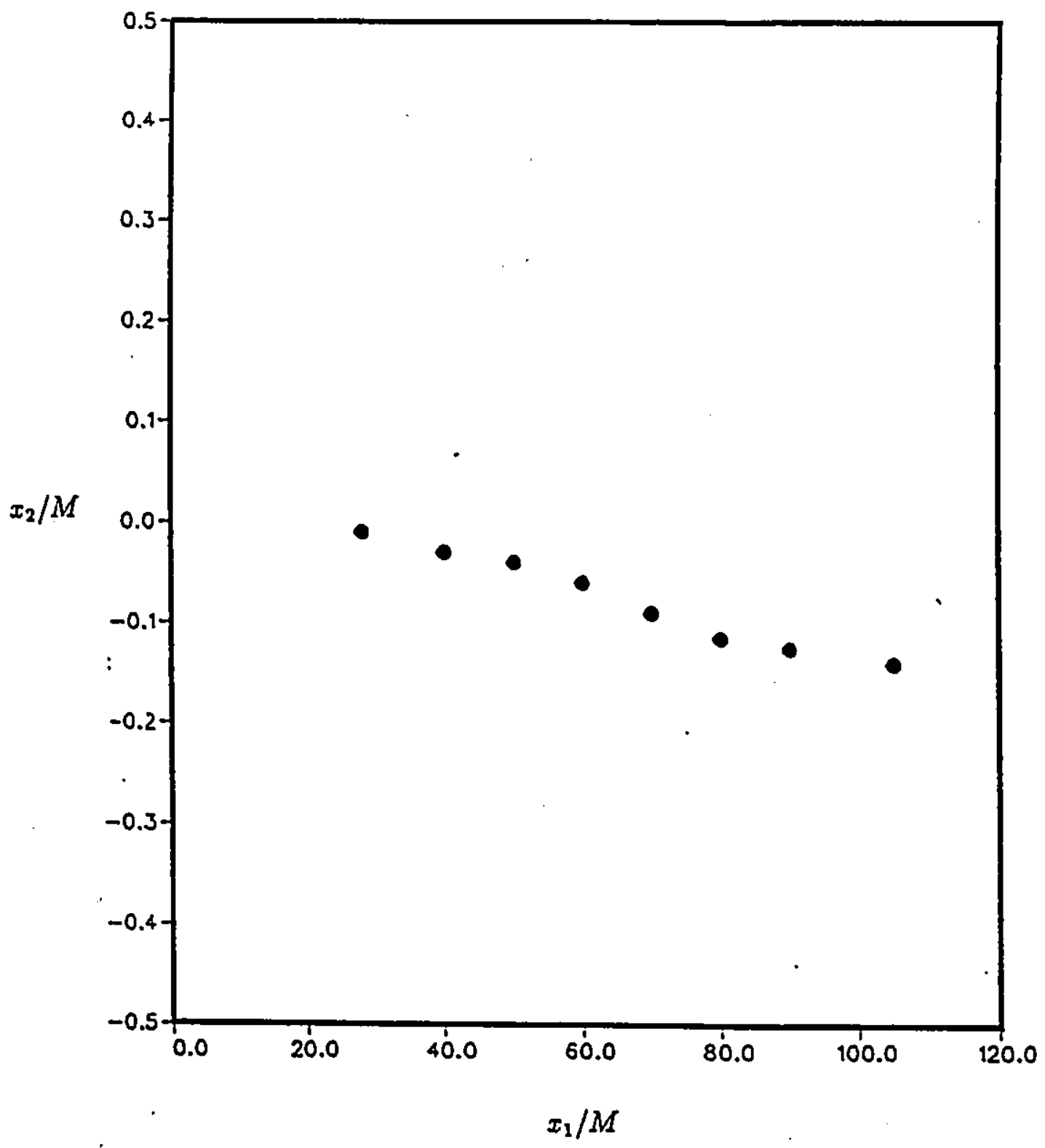

Figure 6.16: Positions of the temperature variance peaks. 


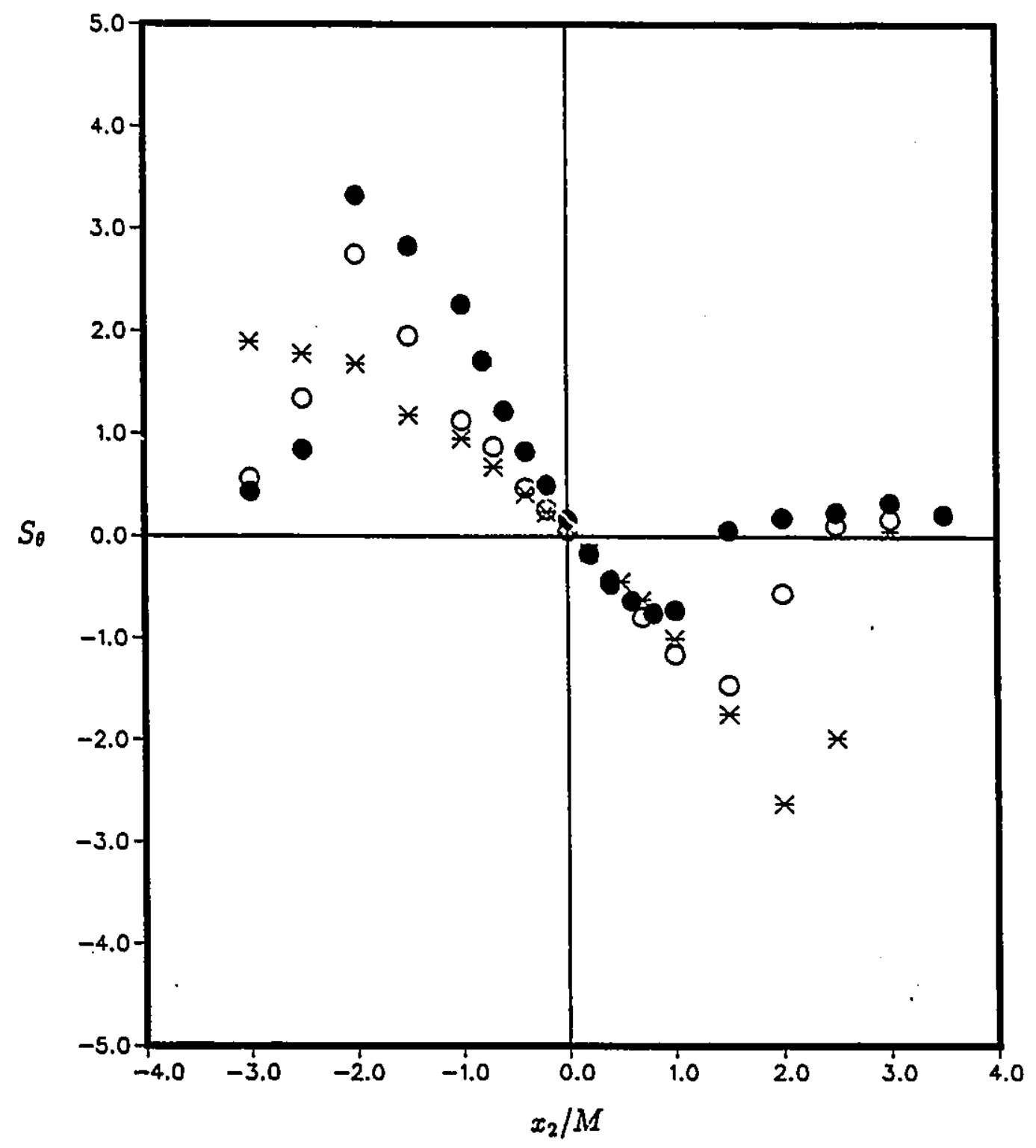

Figure 6.17: Transverse variation of the temperature fluctuation skewness factor. - $x_{1} / M=40 \circ x_{1} / M=60 * x_{1} / M=80$. 

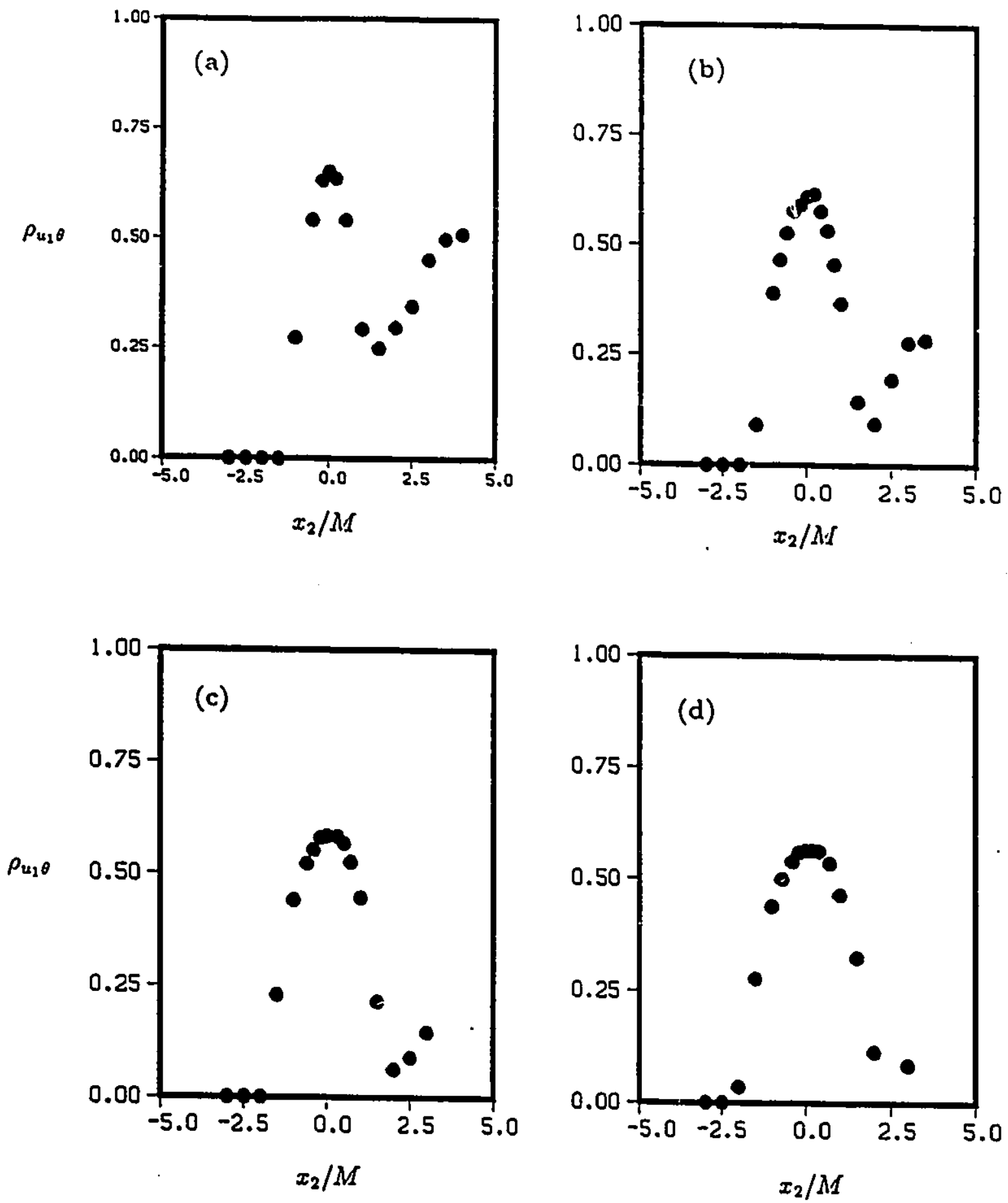

Figure 6.18: Transverse variation of the streamwise heat flux coefficient. (a) $x_{1} / M=28$ (b) $x_{1} / M=40$ (c) $x_{1} / M=50$ (d) $x_{1} / M=60$ (e) $x_{1} / M=70$ (f) $x_{1} / M=80(\mathrm{~g}) x_{1} / M=90(\mathrm{~h}) x_{1} / M I=105$. 

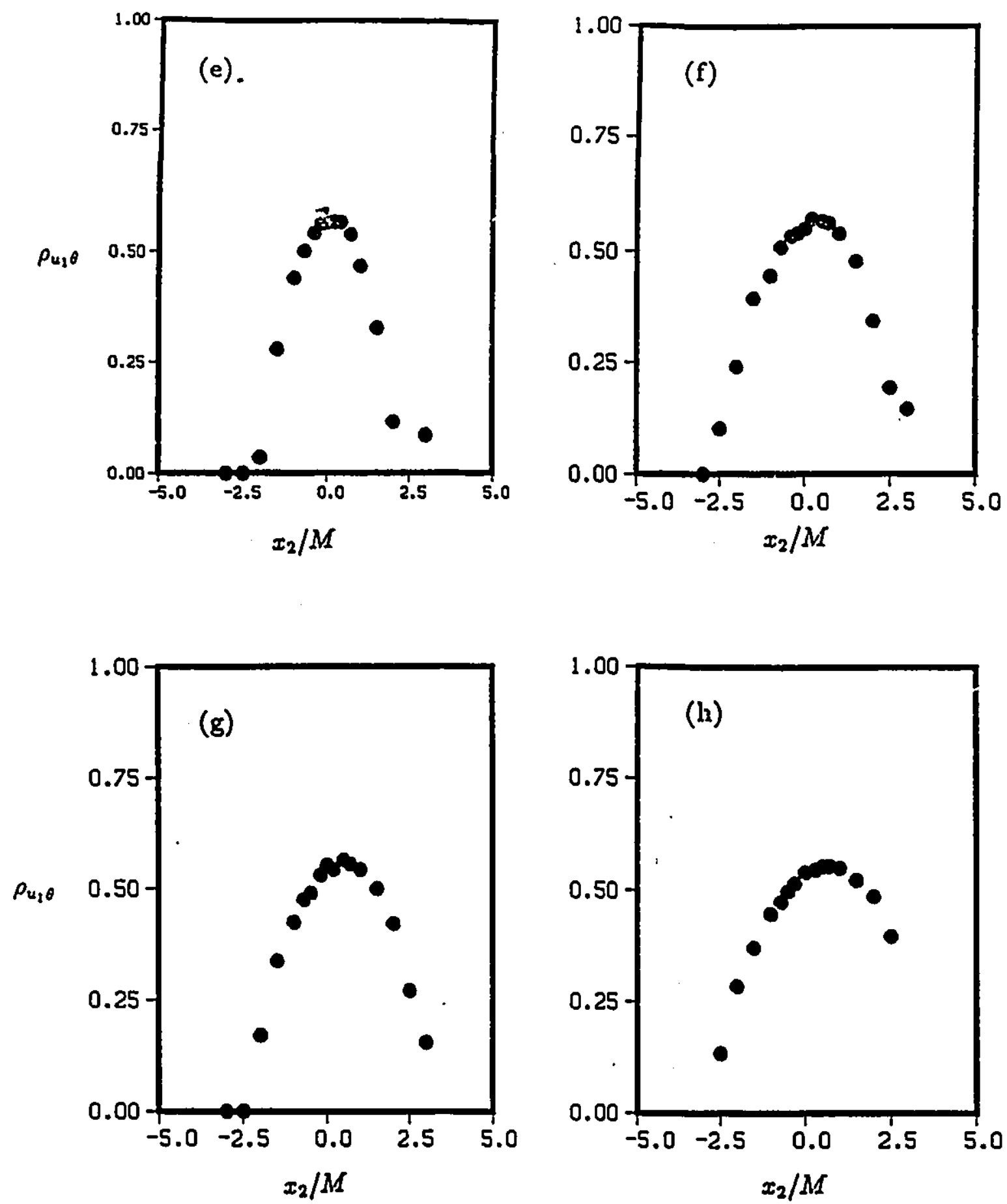

Figure 6.1s. Cont'd. 

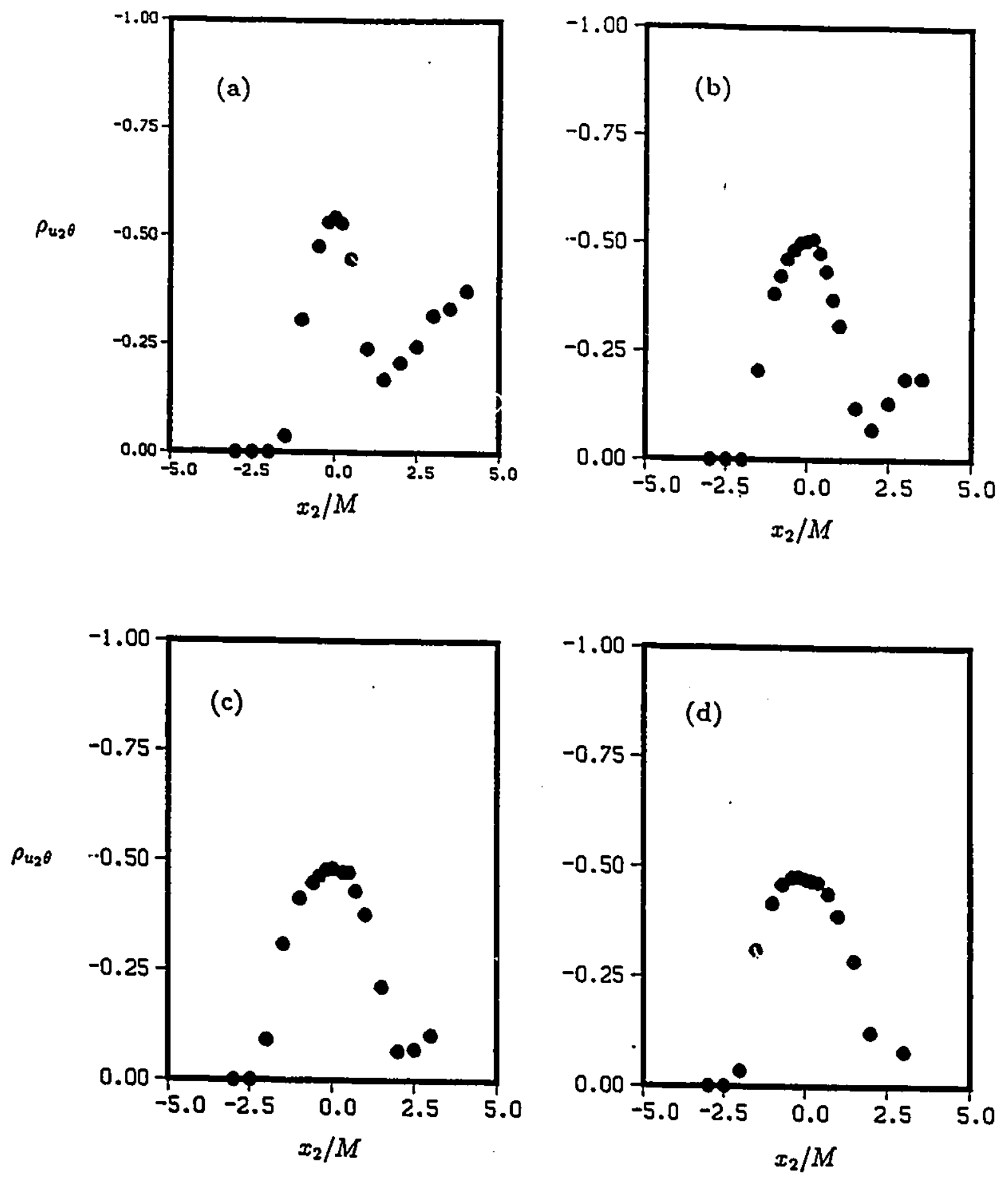

Figure 6.19: Transverse variation of the transverse heat flux coefficient. (a) $x_{1} / M=28$ (b) $x_{1} / M=40$ (c) $x_{1} / M=50$ (d) $x_{1} / M=60$ (e) $x_{1} / M=70$ (f)
$x_{1} / M=80$ (g) $x_{1} / M=90$ (h) $x_{1} / M=105$. 

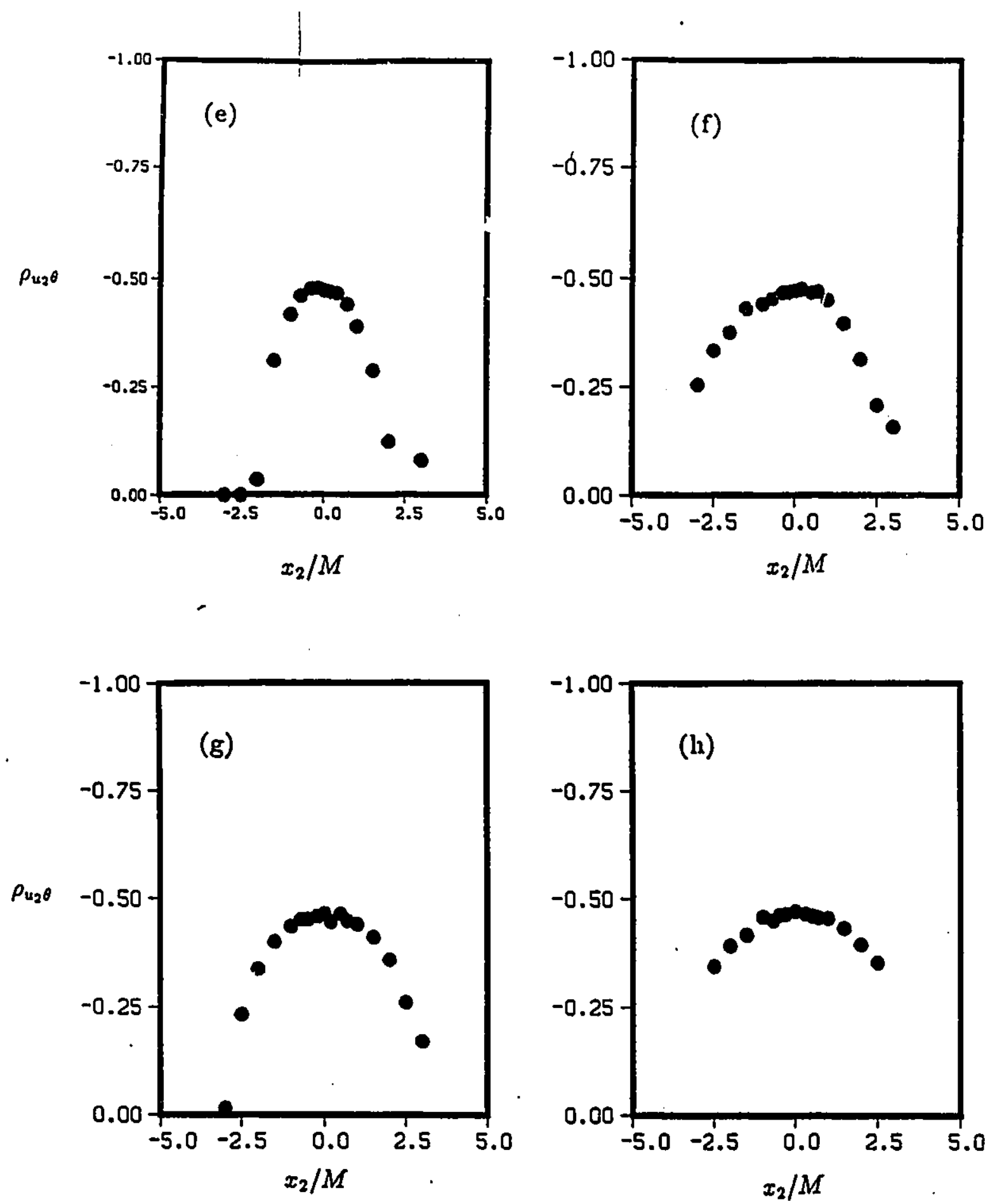

Figure 6.19. Cont'd. 


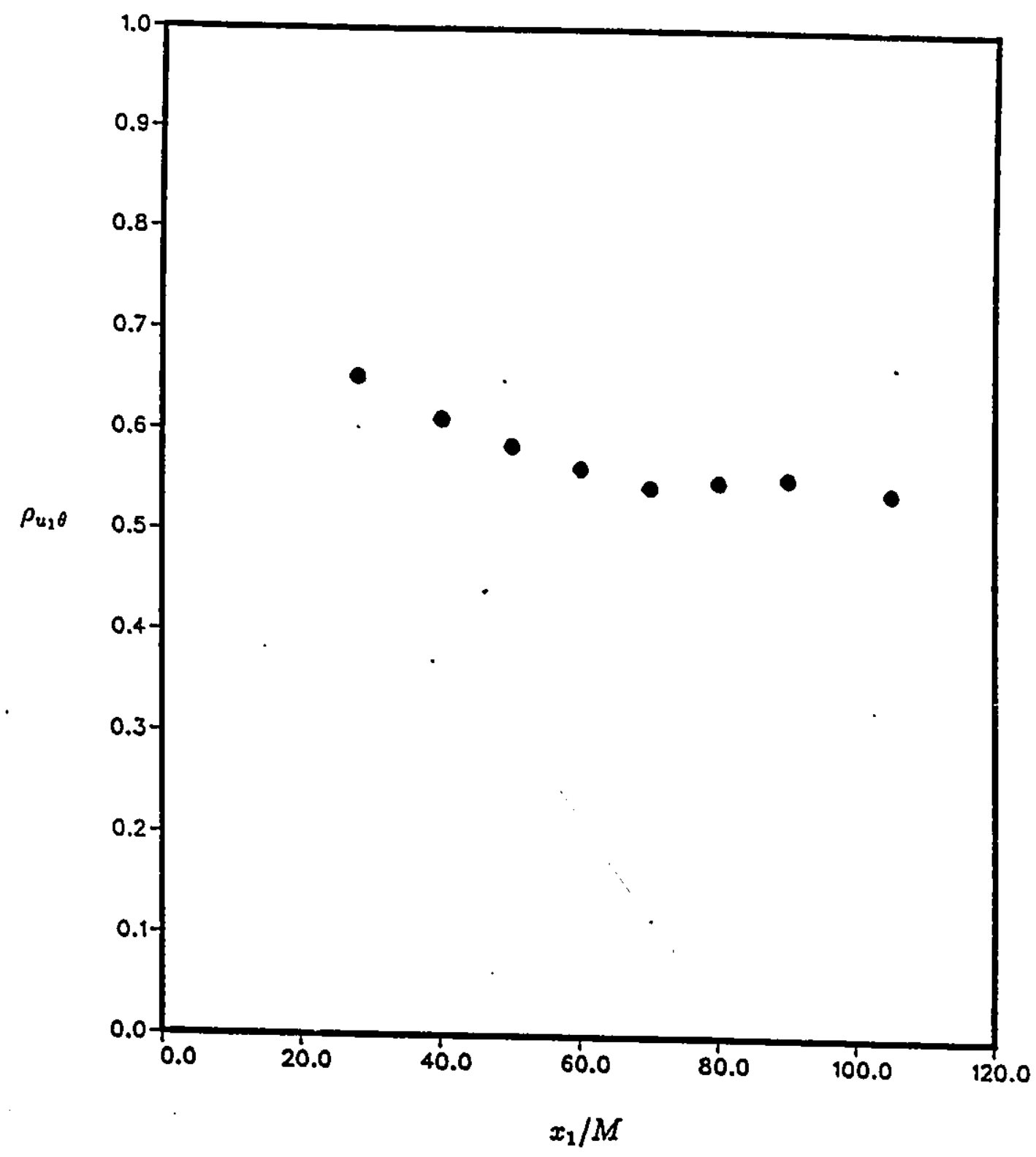

Figure 6.20: Centerline streamivise heat flux coefficient. 


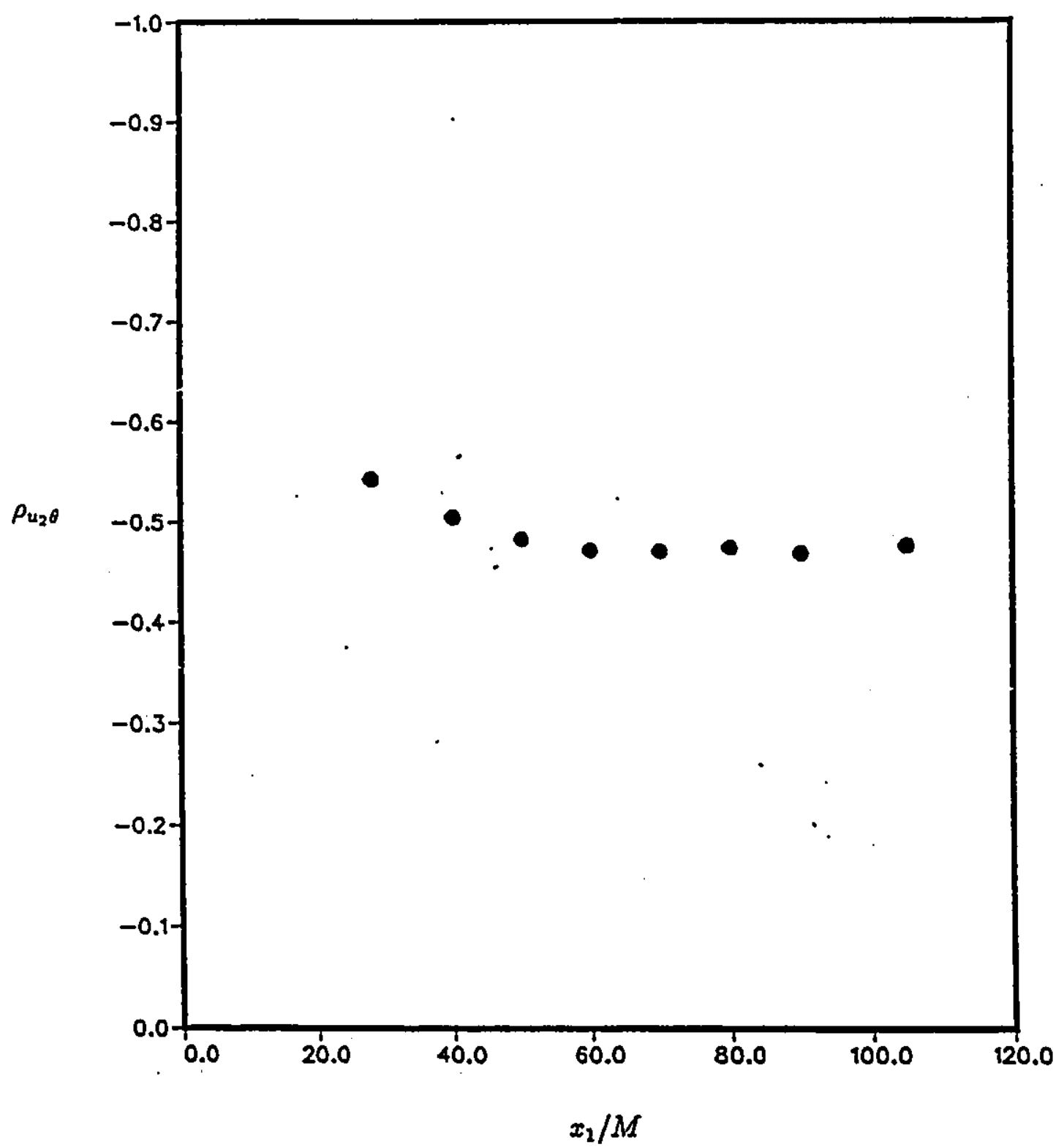

Figure 6.21: Centerline transverse heat flux coefficient. 


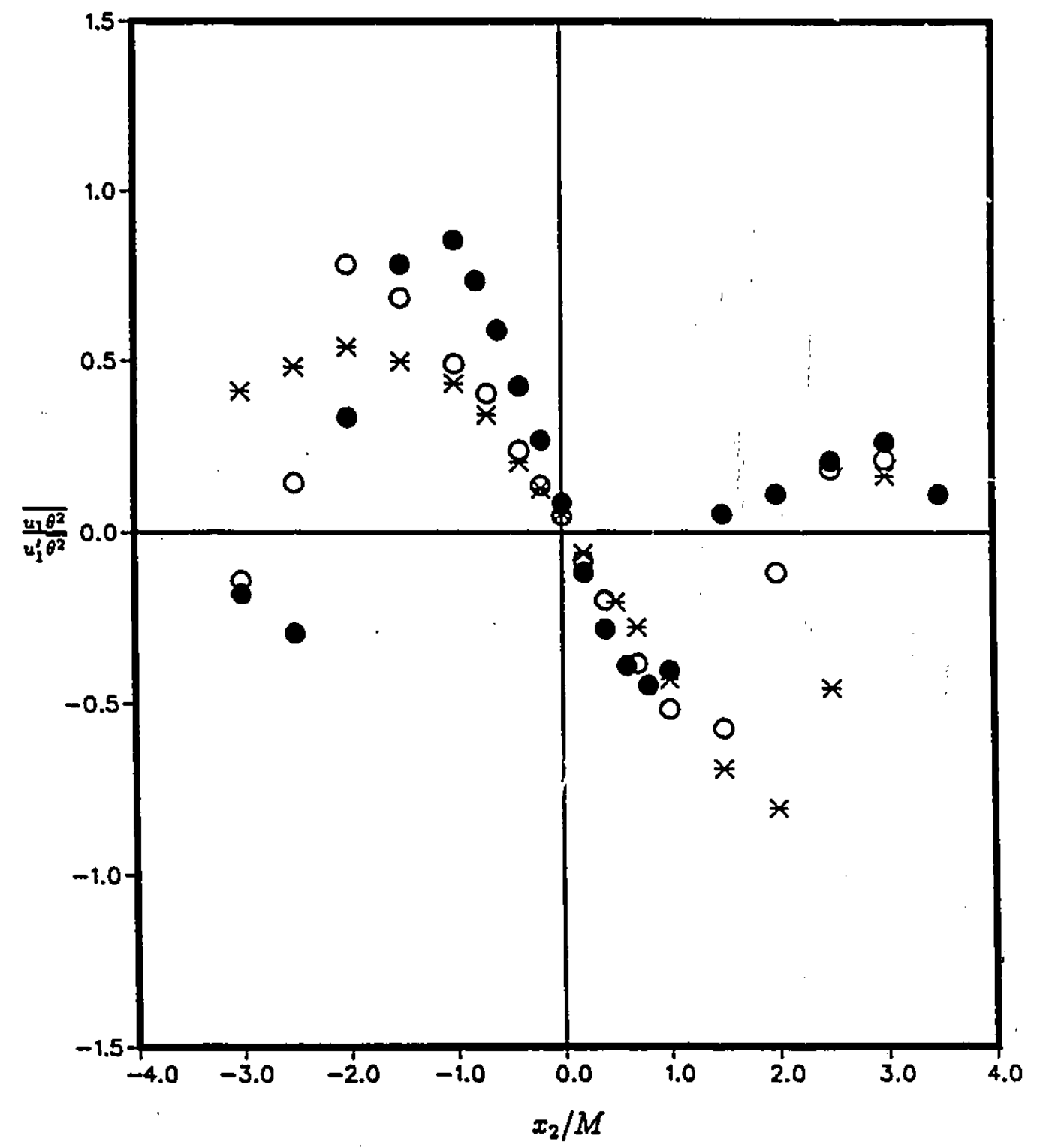

Figure 6.22: Triple correlation coefficient $\frac{\overline{u_{1} \theta^{2}}}{\overline{u_{1}^{\prime} \overline{\theta^{2}}}} \cdot x_{1} / M=40 \circ x_{1} / M=60$ * $x_{1} / M=\varepsilon ?$. 


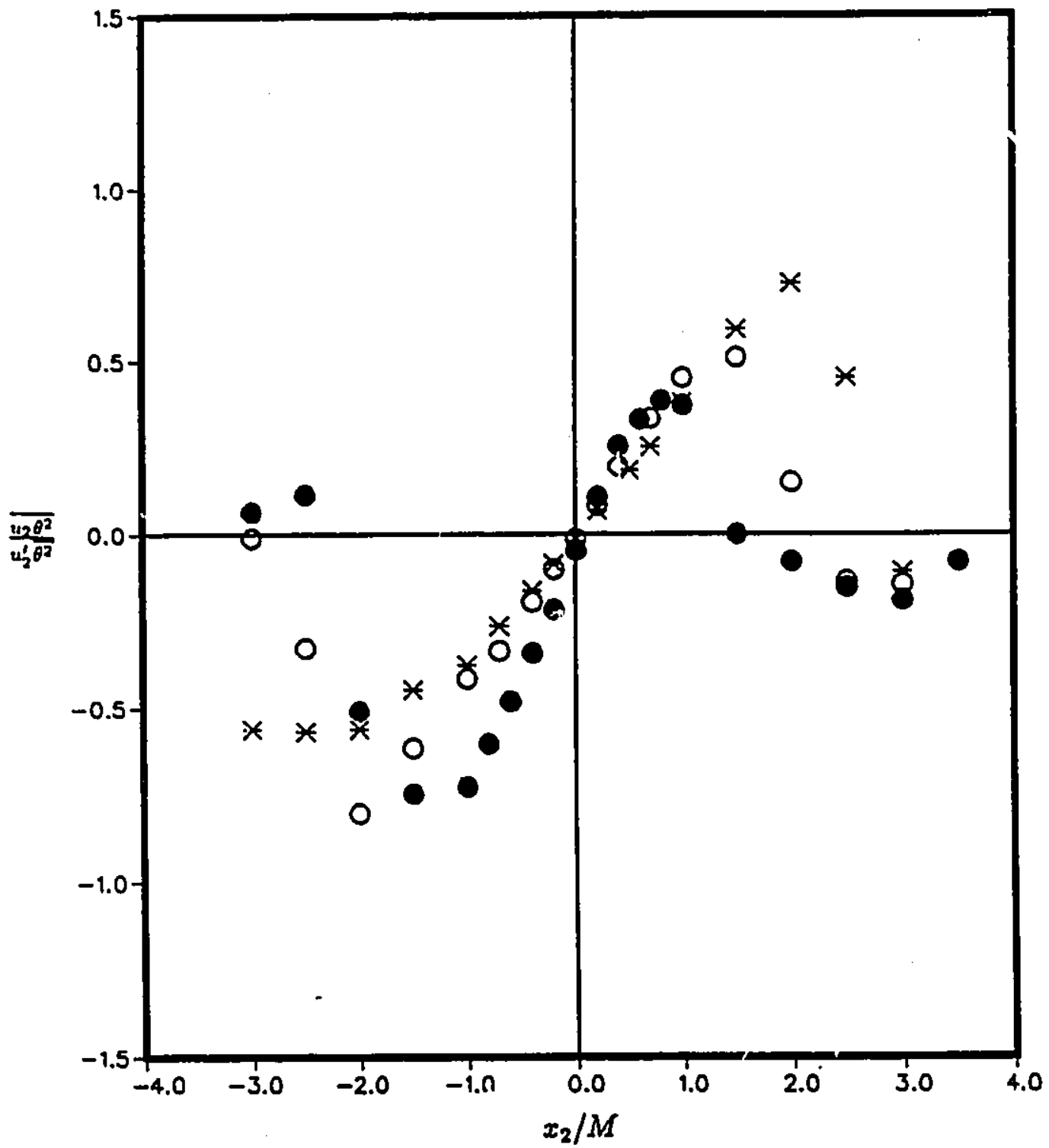

Figure 6.23: Triple correlation coefficient $\frac{\overline{u_{2} \theta^{2}}}{\frac{u_{2}^{\prime} \theta^{2}}{\theta^{2}}} \cdot x_{1} / M=40 \circ x_{1} / M=60 *$ $x_{1} / M=S 0$. 


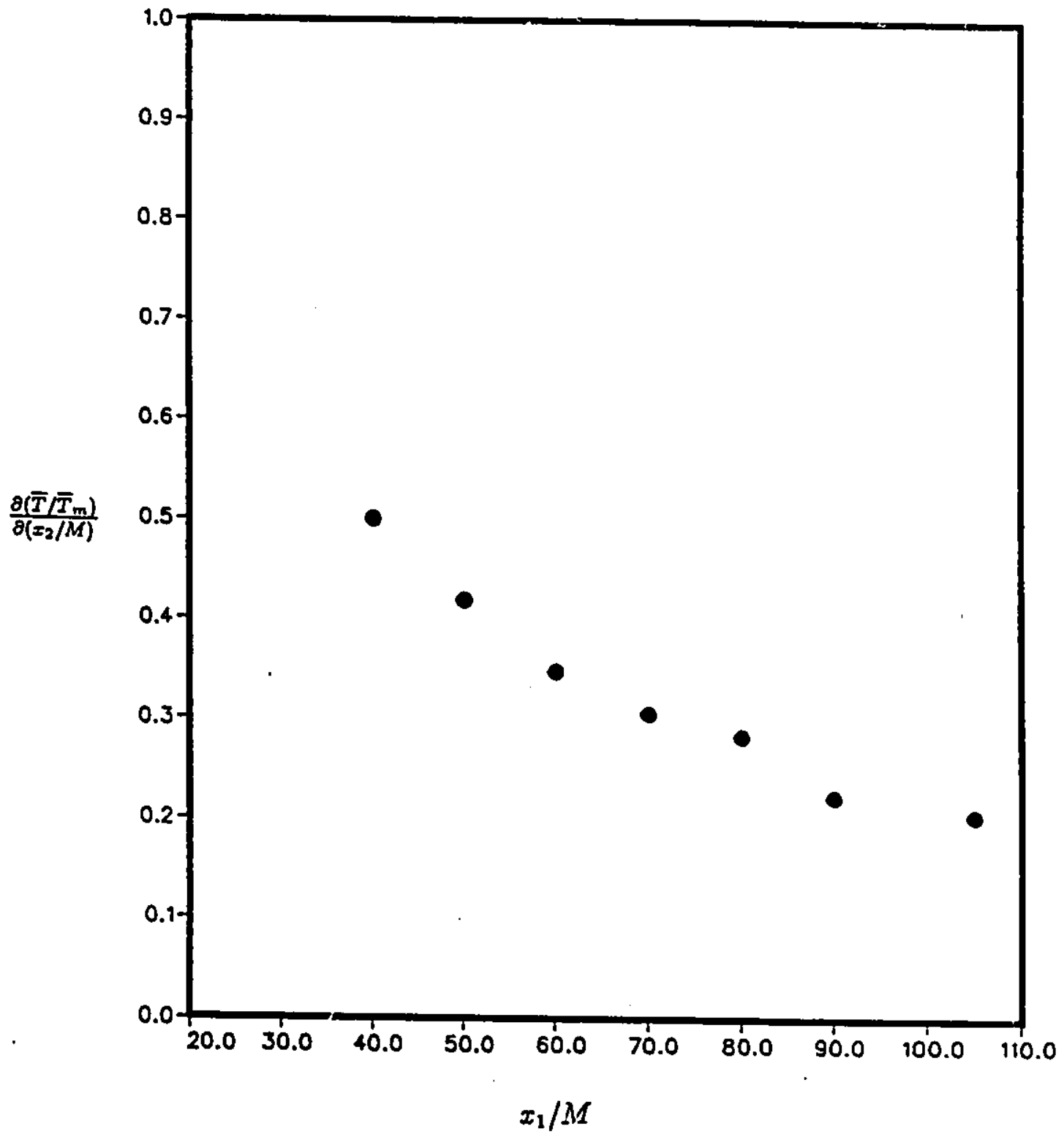

Figure 7.1: Dimensionless maximum temperature gradient. 


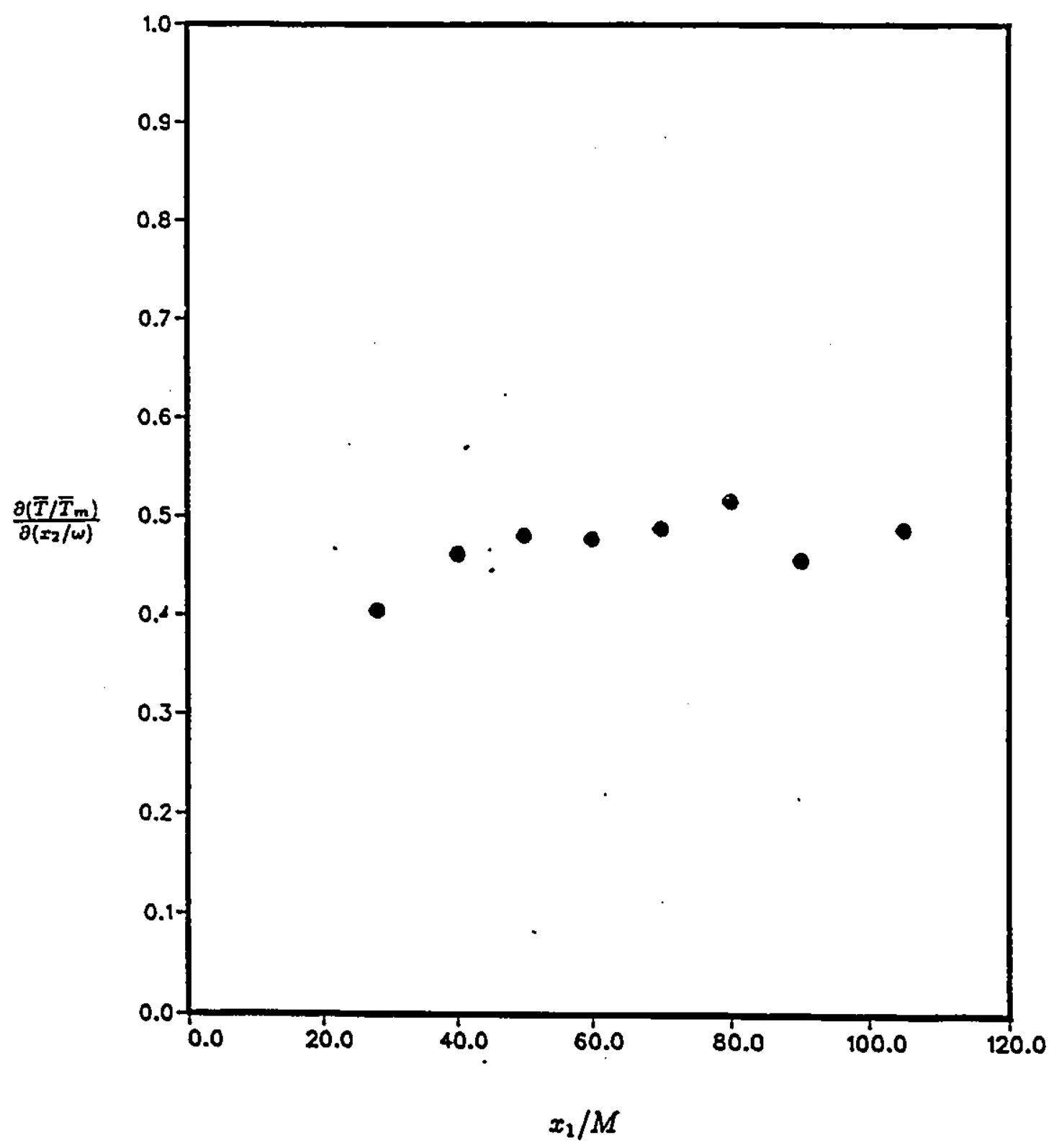

Figure 7.2: Dimensionless maximum temperature gradient. 


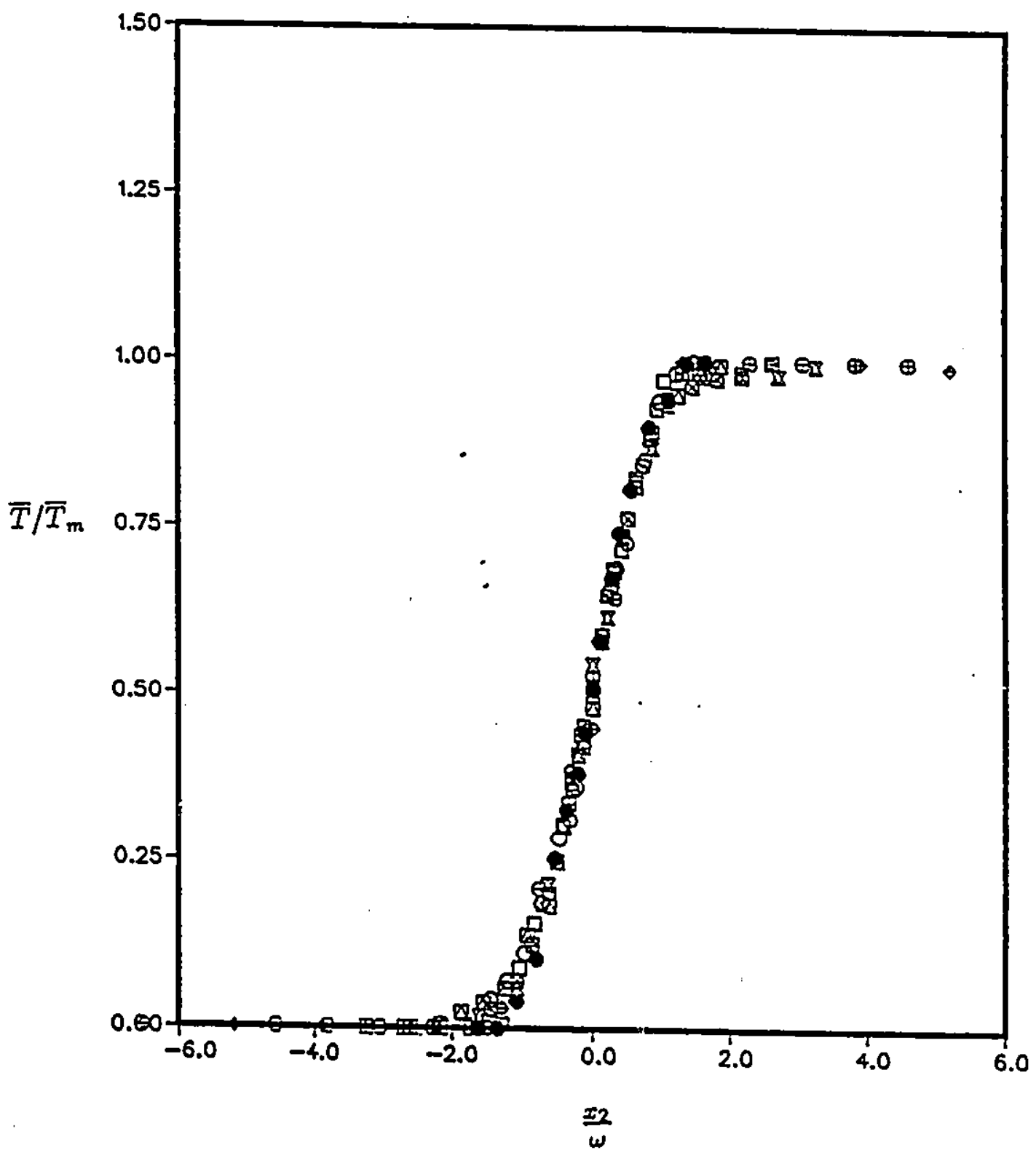

Figure 7.3: Collapse of all mean temperature profiles. 


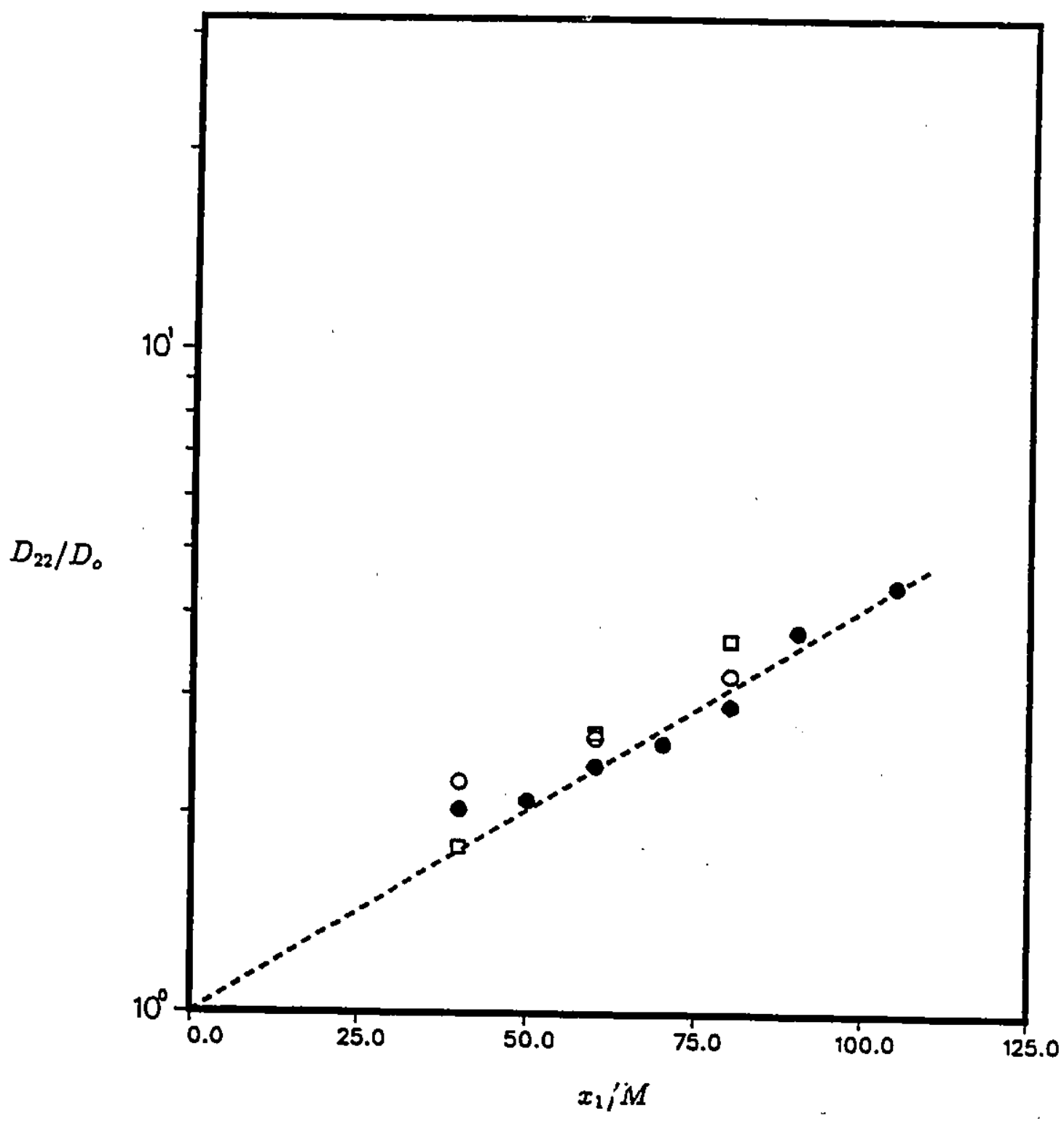

Figure 7.4: Estimates of the turbulent diffusivity, $D_{22}$ • as $\frac{-\overline{\theta u_{2}}}{\partial \bar{T} / \partial x_{2}} ; 0$ as $\frac{-\overline{\theta^{2} u_{2}}}{\partial \bar{\theta}^{2} / \partial x_{2}}$ $\left(x_{2}>0\right) ; \square$ as $\frac{-\overline{\theta^{2} u_{2}}}{\partial \theta^{2} / \partial x_{2}}\left(x_{2}<0\right) ;----$ equation(31). 


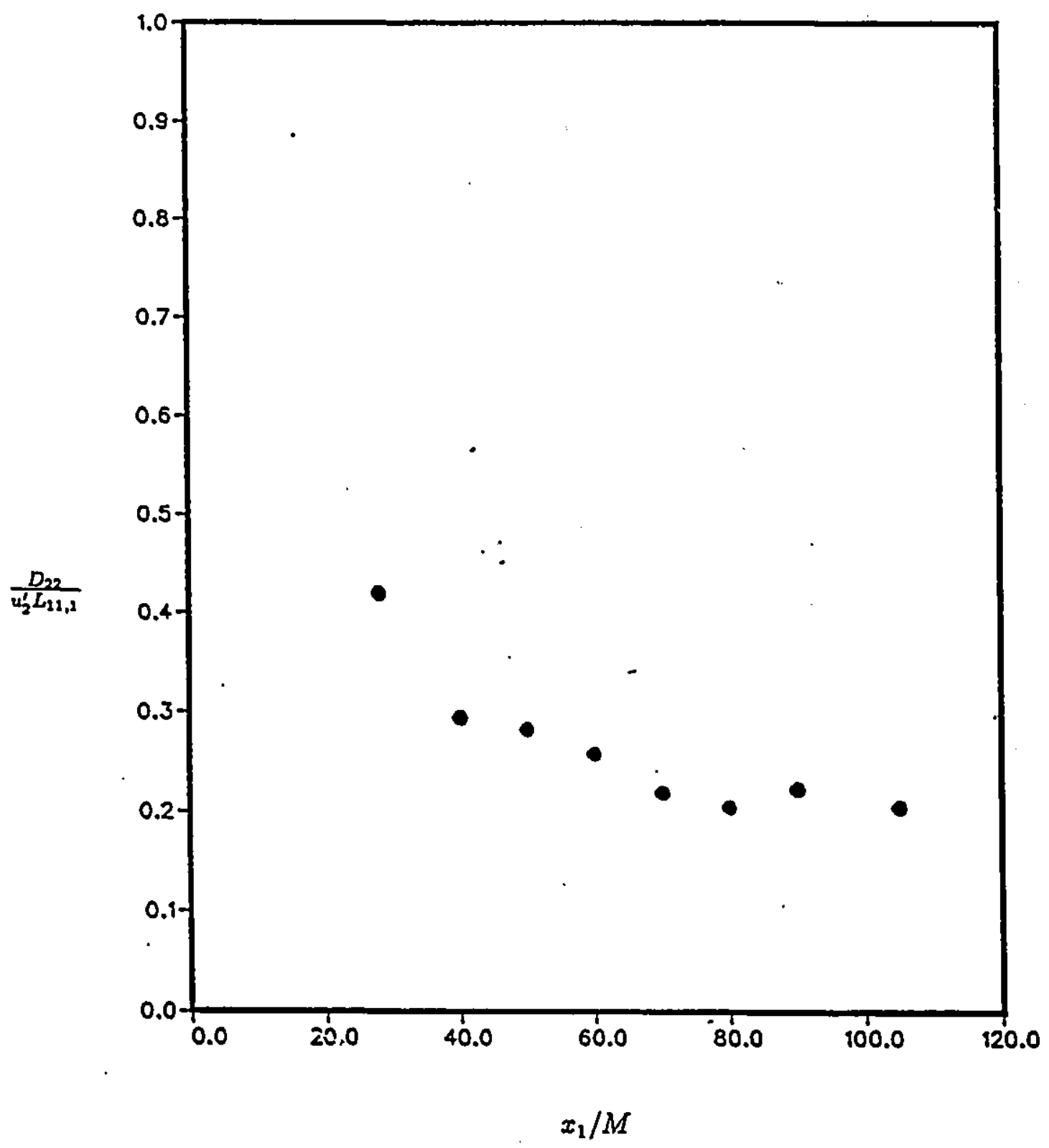

Figure 7.5: Turbulent diffusivity normalized with Eulerian scales. 


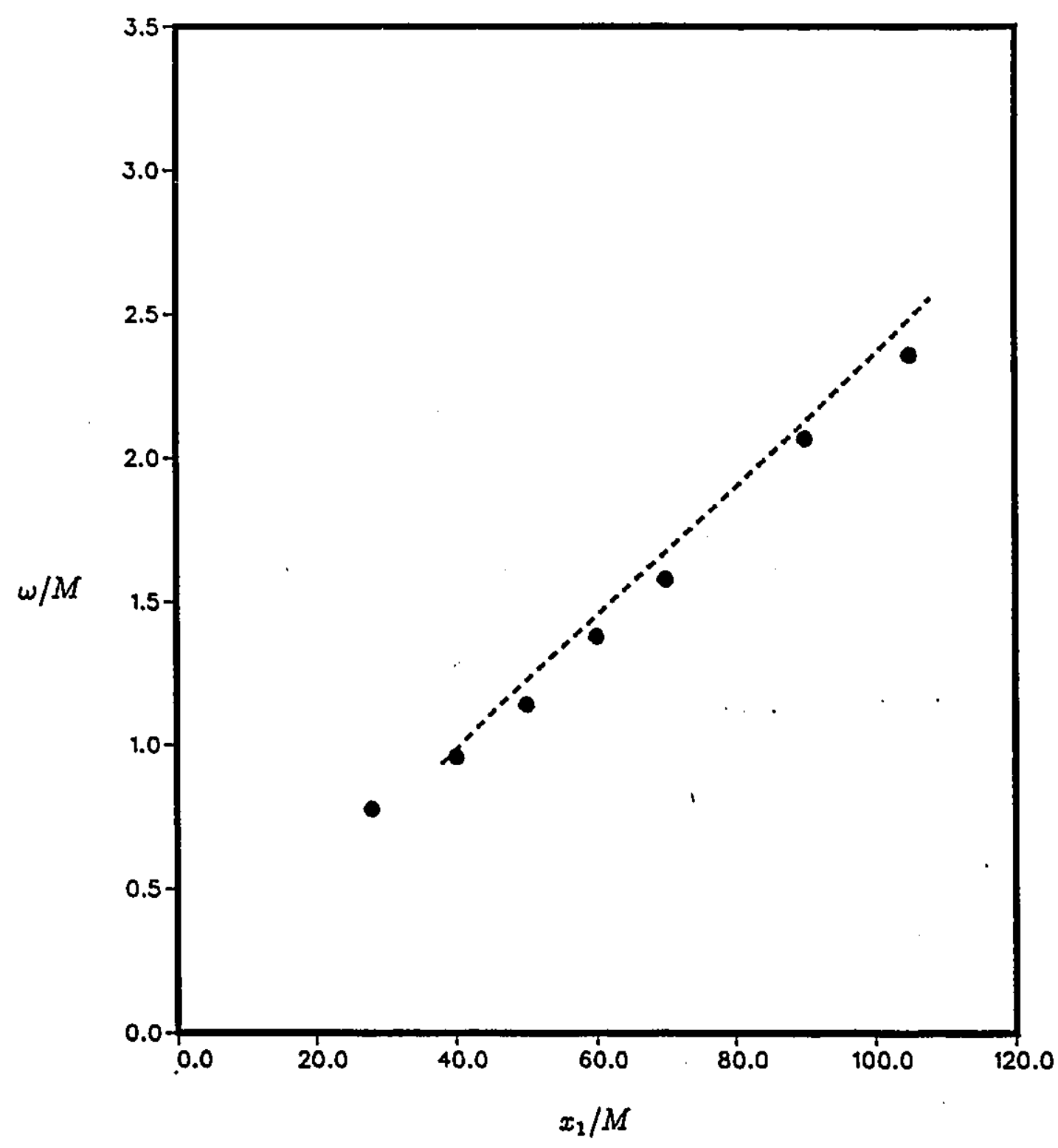

Figure 7.6: Comparison of width with the approximate solution. : measured -.-- equation(7.7). 


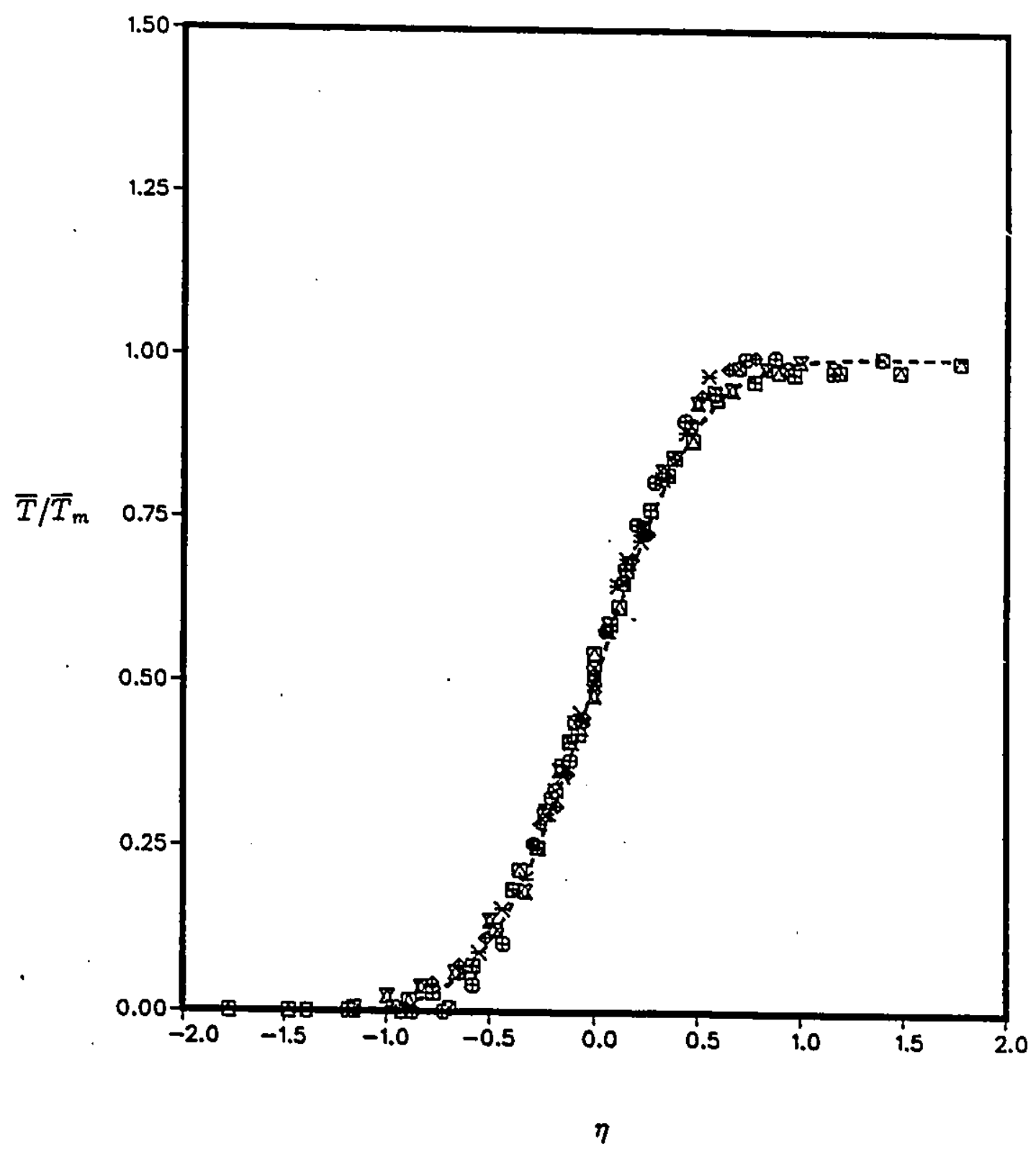

Figure 7.7: Mean temperature profiles. $\Delta \frac{x_{1}}{M}=40 . \otimes \frac{x_{1}}{M}=50 . \oplus \frac{x_{1}}{M}=60 \mathbb{Z} \frac{x_{1}}{M}=70$. $\oplus \frac{x_{1}}{M}=80 . \div \frac{x_{1}}{M}=90-\frac{x_{1}}{M}=105 \ldots$ solution(4.13). 


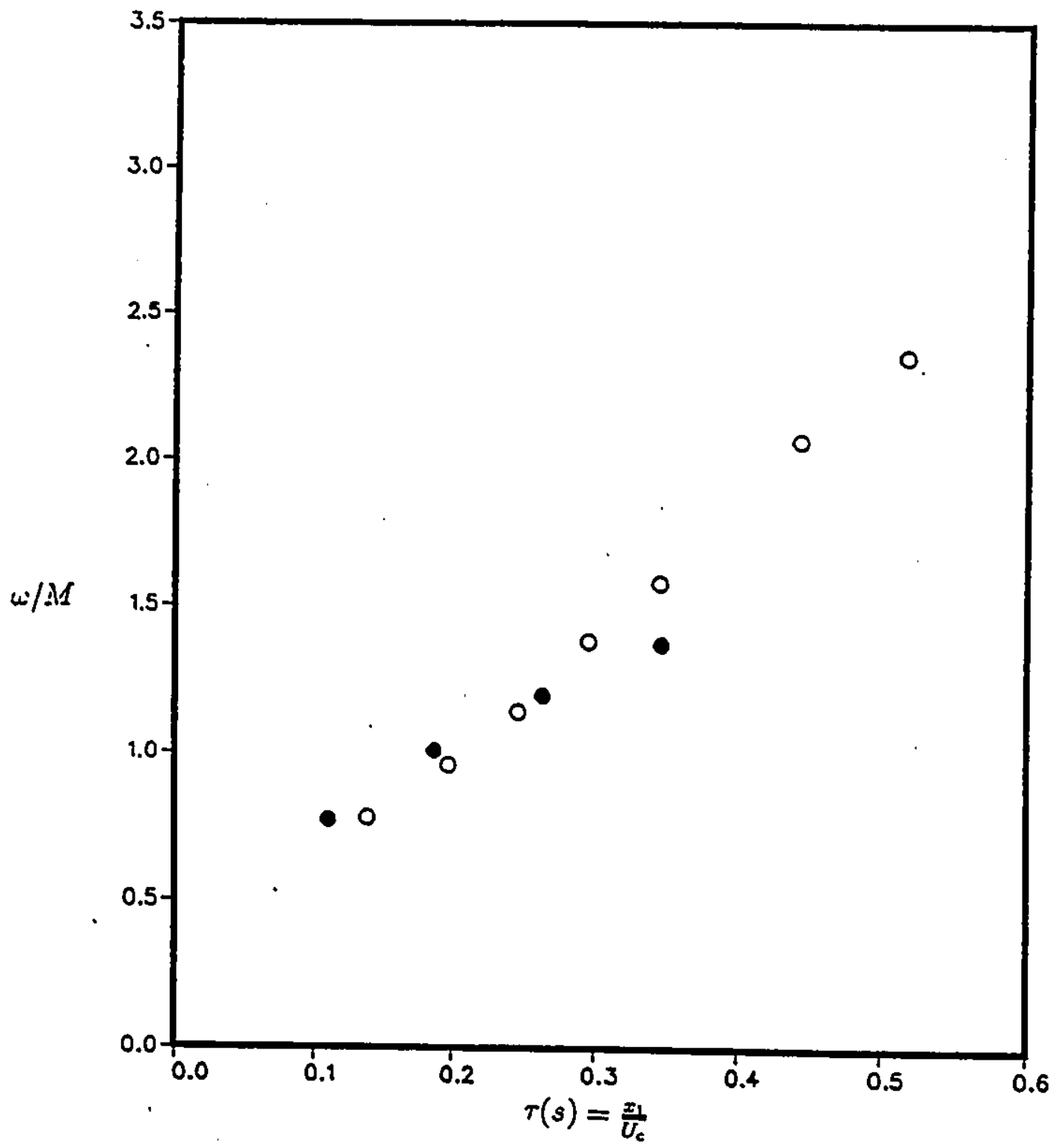

Figure 7.8: Comparison of thermal mixing layer width in isotropic $(\bullet)$ and uniformly sheared flows(0). 


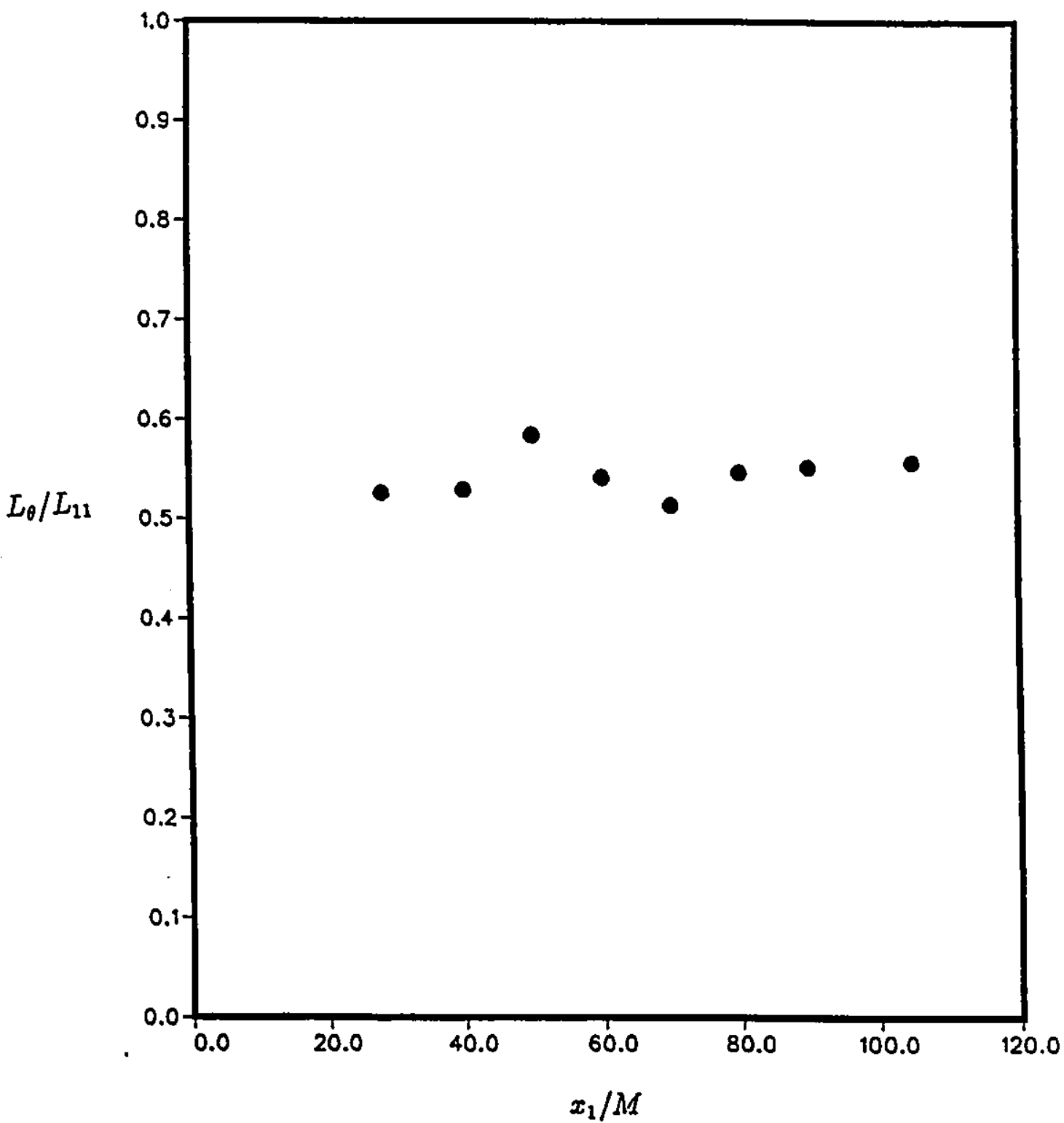

Figure 7.9: Ratio of temperature to velocity streamwise length scales. 


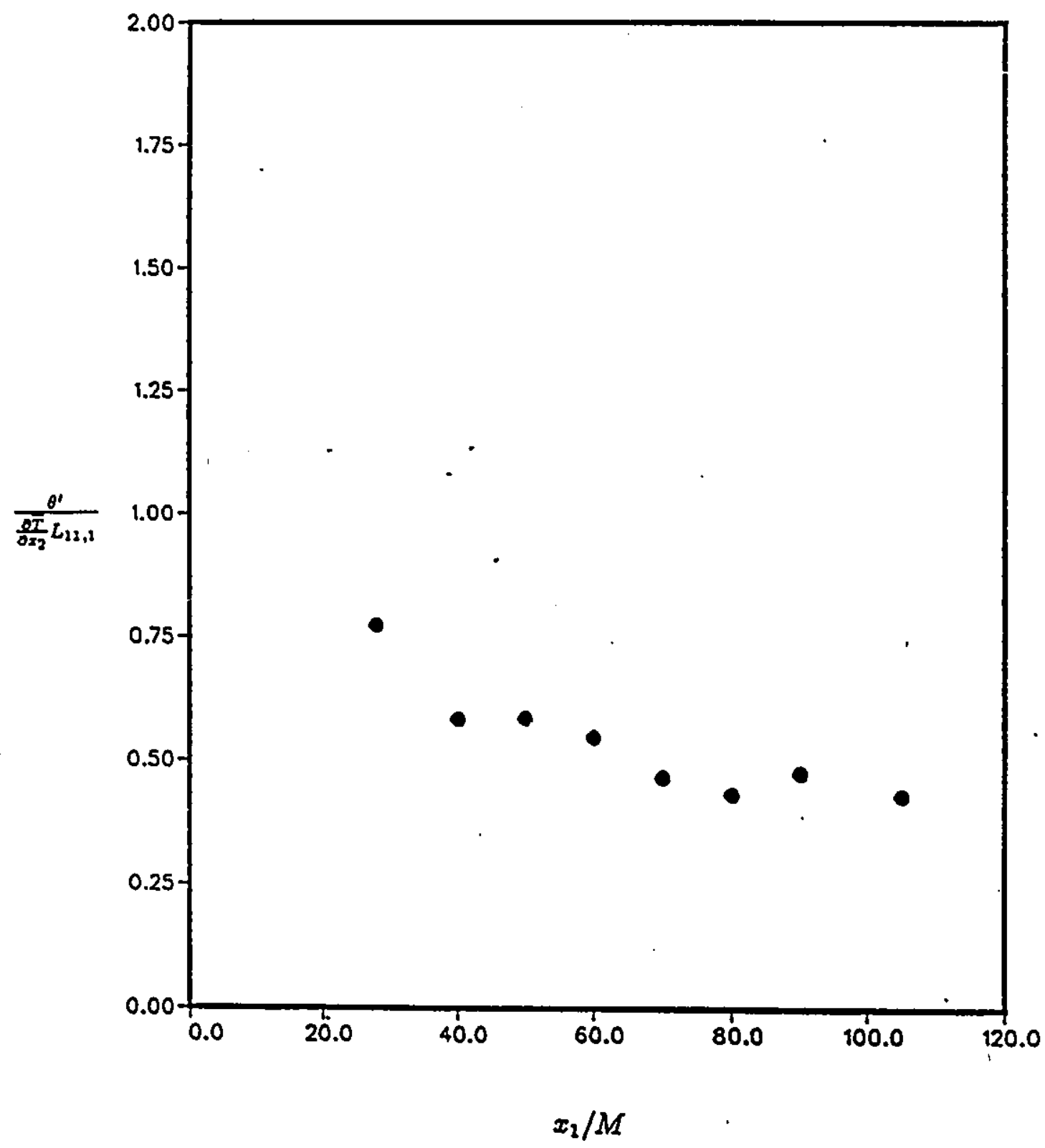

Figure 7.10: Normalized peak r.m.s. temperature fluctuations. 
Appendix A

\section{Tables Containing Measured and Estimated Quantities}




\begin{tabular}{|c|c|}
\hline$\stackrel{\Im}{\widetilde{\Xi}}$ & 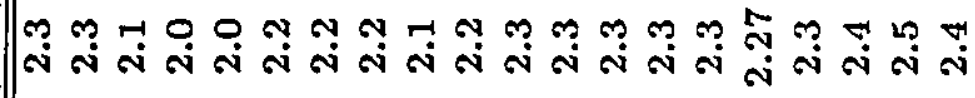 \\
\hline & 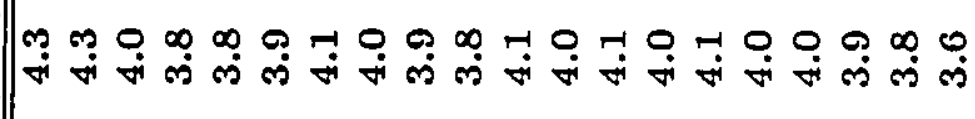 \\
\hline & ف일 \\
\hline & | \\
\hline (i) & W \\
\hline I5 & 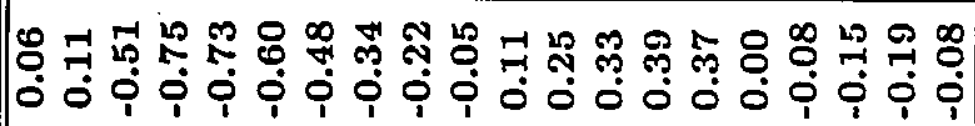 \\
\hline $\begin{array}{l}18 \\
2\end{array}$ & 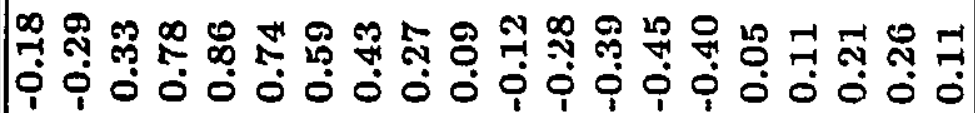 \\
\hline$\stackrel{5}{2}$ & 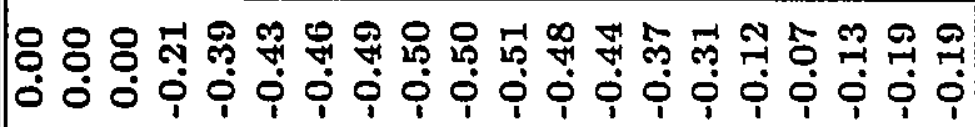 \\
\hline 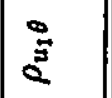 & 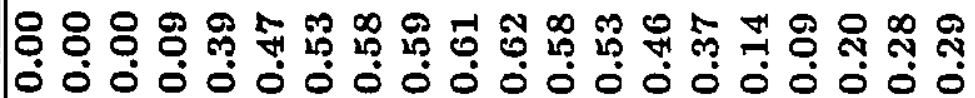 \\
\hline & 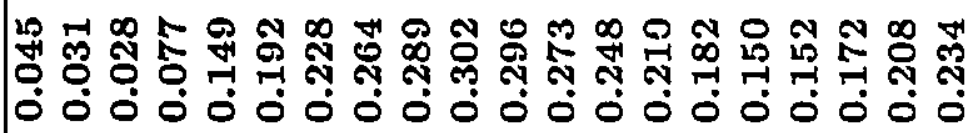 \\
\hline & \\
\hline
\end{tabular}
F

개

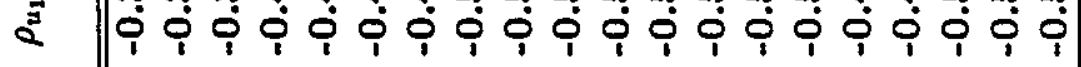
₹ E

= क \# E

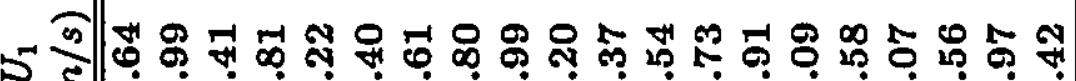

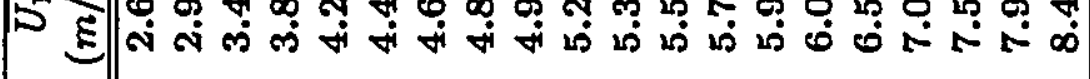
กิบ

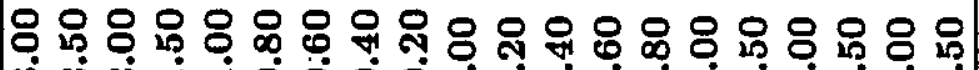
भं 


\begin{tabular}{|c|c|c|c|c|c|c|c|c|}
\hline$\frac{\Sigma_{2}}{\bar{T}^{\prime}}$ & $\begin{array}{c}\overline{U_{1}} \\
(\mathrm{~m} / \mathrm{s})\end{array}$ & $\begin{array}{c}u_{1}^{\prime} \\
(\mathrm{m} / \mathrm{s})\end{array}$ & $\begin{array}{c}u_{2}^{\prime} \\
(\mathrm{m} / \mathrm{s})\end{array}$ & $\rho_{u_{1} u_{2}}$ & $\begin{array}{c}\bar{T} \\
\left({ }^{0} \mathrm{~K}\right)\end{array}$ & $\begin{array}{c}\theta^{\prime} \\
\left({ }^{0} \mathrm{~K}\right)\end{array}$ & $\rho_{u_{1} \theta}$ & $\rho_{u_{2} 0}$ \\
\hline \hline-3.00 & 2.68 & 0.35 & 0.22 & -0.36 & 0.00 & 0.040 & 0.00 & 0.00 \\
-2.50 & 3.05 & 0.40 & 0.24 & -0.40 & 0.00 & 0.035 & 0.00 & 0.00 \\
-2.00 & 3.37 & 0.39 & 0.25 & -0.39 & 0.00 & 0.042 & 0.00 & -0.09 \\
-1.50 & 3.80 & 0.42 & 0.27 & -0.42 & 0.01 & 0.087 & 0.23 & -0.31 \\
-1.00 & 4.23 & 0.42 & 0.28 & -0.44 & 0.14 & 0.156 & 0.44 & -0.41 \\
-0.60 & 4.61 & 0.44 & 0.28 & -0.46 & 0.28 & 0.211 & 0.52 & -0.45 \\
-0.40 & 4.79 & 0.44 & 0.28 & -0.47 & 0.38 & 0.229 & 0.55 & -0.47 \\
-0.20 & 4.99 & 0.45 & 0.29 & -0.49 & 0.49 & 0.247 & 0.58 & -0.48 \\
0.00 & 5.17 & 0.44 & 0.29 & -0.50 & 0.58 & 0.260 & 0.58 & -0.48 \\
0.30 & 5.45 & 0.44 & 0.29 & -0.50 & 0.73 & 0.249 & 0.58 & -0.47 \\
0.50 & 5.62 & 0.43 & 0.28 & -0.51 & 0.82 & 0.232 & 0.57 & -0.47 \\
0.70 & 5.82 & 0.42 & 0.28 & -0.50 & 0.91 & 0.205 & 0.53 & -0.43 \\
1.00 & 6.11 & 0.41 & 0.27 & -0.50 & 1.00 & 0.159 & 0.45 & -0.38 \\
1.50 & 6.60 & 0.39 & 0.26 & -0.50 & 1.10 & 0.103 & 0.21 & -0.21 \\
2.00 & 7.09 & 0.36 & 0.25 & -0.48 & 1.10 & 0.093 & 0.06 & -0.07 \\
2.50 & 7.55 & 0.34 & 0.24 & -0.50 & 1.10 & 0.109 & 0.09 & -0.07 \\
3.00 & 8.01 & 0.33 & 0.23 & -0.51 & 1.12 & 0.133 & 0.15 & -0.10 \\
\hline
\end{tabular}

Table A.2: Measurements at $x_{1} / M=50$ 


\begin{tabular}{|c|c|}
\hline$\stackrel{\overbrace{}}{\overparen{\Xi}}$ & 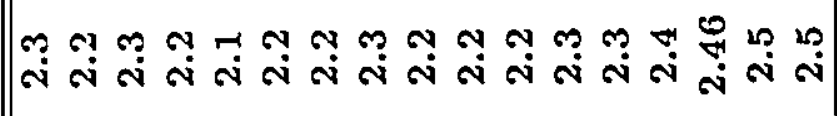 \\
\hline & 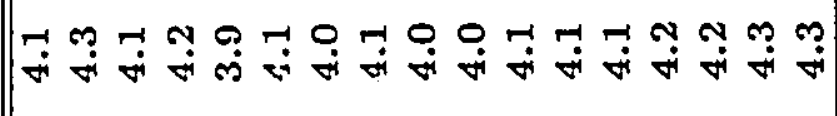 \\
\hline $\bar{్}$ & 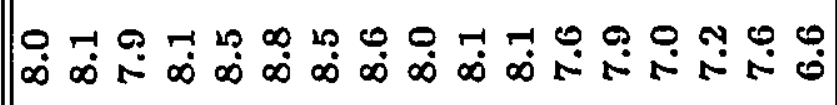 \\
\hline$=\overparen{E}$ & | \\
\hline क & 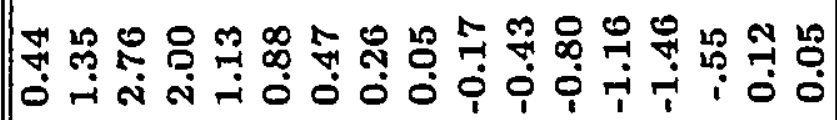 \\
\hline$e^{10}$ & 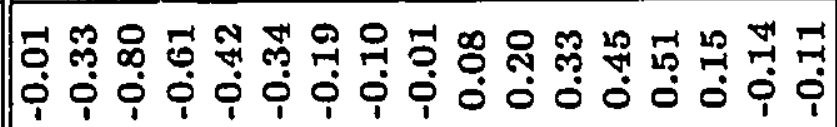 \\
\hline$e^{15}$ & 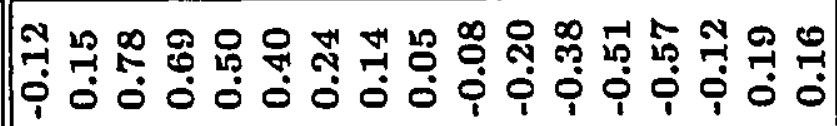 \\
\hline i & 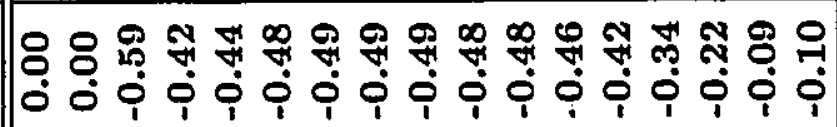 \\
\hline$\stackrel{5}{2}$ & 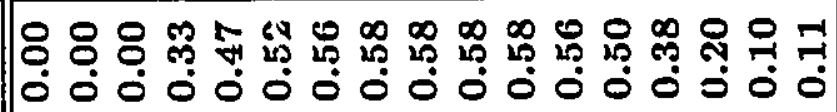 \\
\hline & 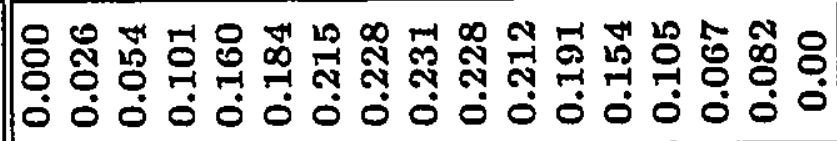 \\
\hline Er $\frac{\text { is }}{2}$ & 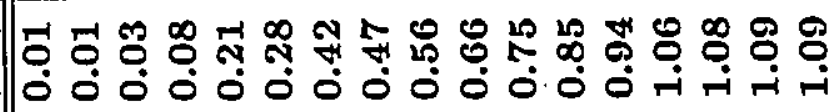 \\
\hline$\underset{3}{3}$ & 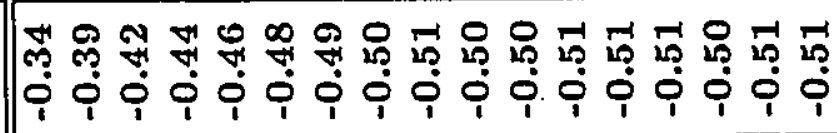 \\
\hline$\approx \frac{D}{E}$ & 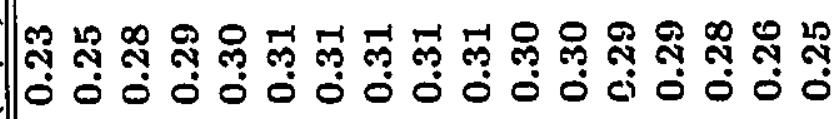 \\
\hline$=-\frac{5}{E}$ & 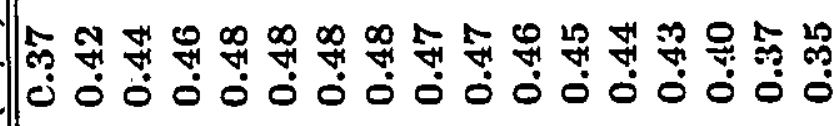 \\
\hline$-\frac{\infty}{E}$ & 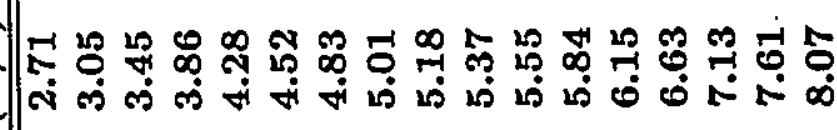 \\
\hline માટ & 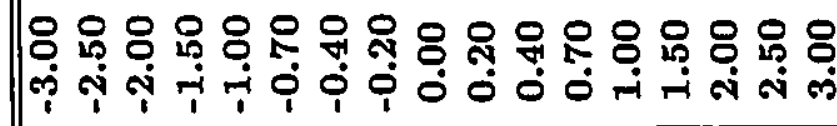 \\
\hline
\end{tabular}




\begin{tabular}{|c|c|}
\hline$\stackrel{N}{\stackrel{\Xi}{\Xi}}$ & 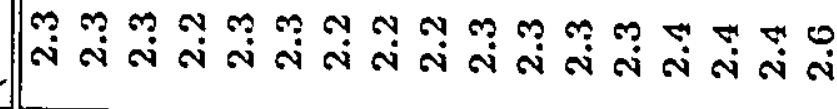 \\
\hline 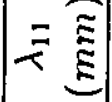 & 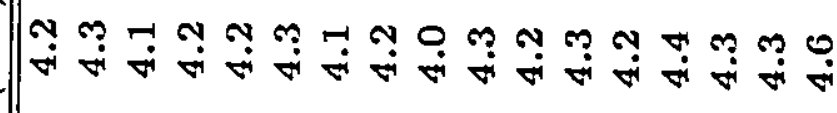 \\
\hline & 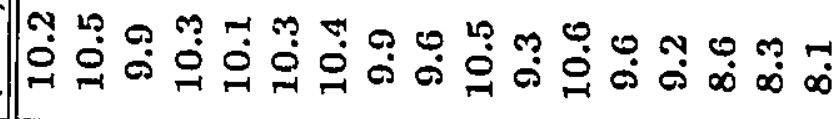 \\
\hline & 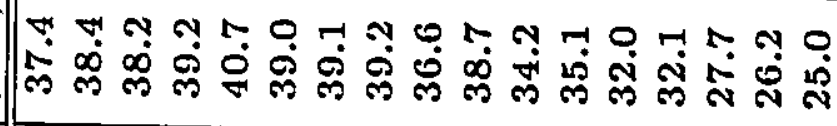 \\
\hline (i) & 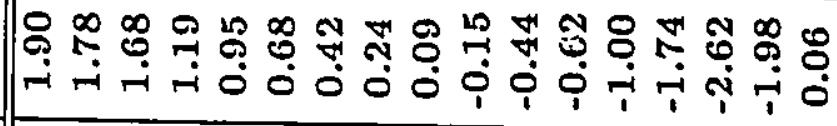 \\
\hline & 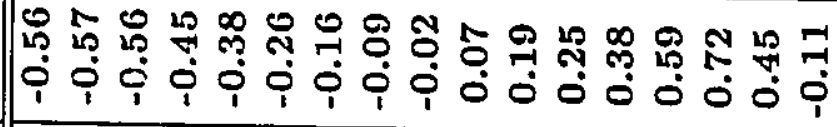 \\
\hline & 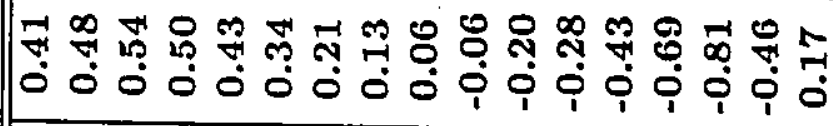 \\
\hline 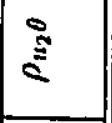 & 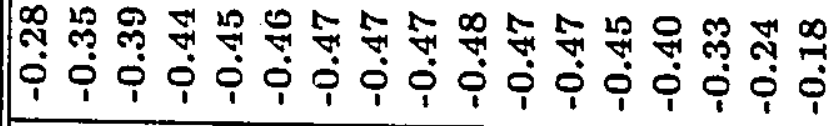 \\
\hline 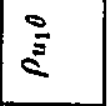 & 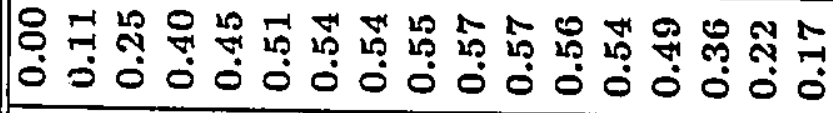 \\
\hline & 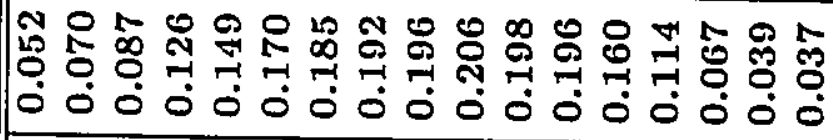 \\
\hline & 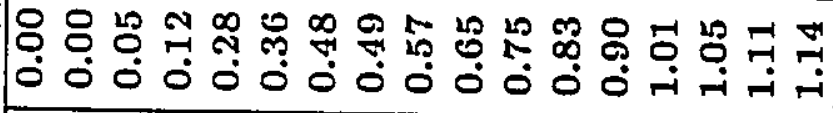 \\
\hline$\stackrel{\Xi}{\equiv}$ & 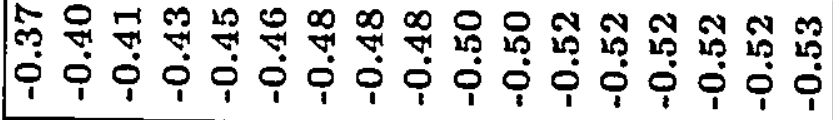 \\
\hline & 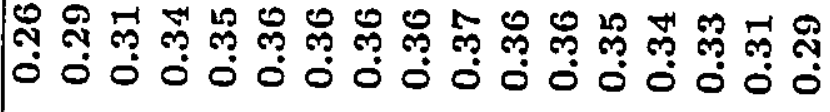 \\
\hline & 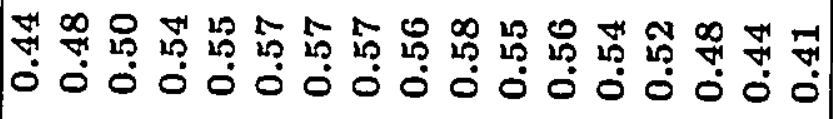 \\
\hline & 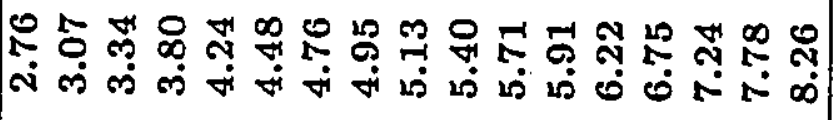 \\
\hline 12 & 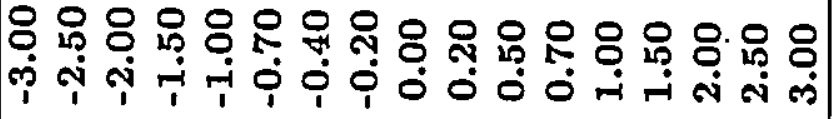 \\
\hline
\end{tabular}




\begin{tabular}{|c|c|c|c|c|c|c|c|c|}
\hline$\frac{52}{M}$ & $\begin{array}{c}\overline{U_{1}} \\
(\mathrm{~m} / \mathrm{s})\end{array}$ & $\begin{array}{c}u_{1} \\
(\mathrm{~m} / \mathrm{s})\end{array}$ & $\begin{array}{c}u_{2} \\
(m / s)\end{array}$ & $\rho_{u_{1} u_{2}}$ & $\begin{array}{c}\bar{T} \\
\left({ }^{\circ}{ }^{\prime}\right)\end{array}$ & $\begin{array}{c}\theta^{\prime} \\
\left({ }^{\circ} I^{\prime \prime}\right)\end{array}$ & $\rho_{u_{1} \theta}$ & $\rho_{u_{2} \theta}$ \\
\hline-3.00 & 2.72 & 0.46 & $\overline{0.27}$ & -0.37 & 0.05 & 0.047 & 0.00 & 0.02 \\
\hline-2.50 & 3.04 & 0.49 & 0.30 & -0.37 & 0.08 & 0.059 & 0.00 & -0.23 \\
\hline-2.00 & 3.41 & 0.52 & 0.33 & -0.41 & 0.12 & 0.083 & 0.17 & -0.34 \\
\hline-1.50 & 3.74 & 0.56 & 0.35 & -0.43 & 0.21 & 0.113 & 0.34 & -0.40 \\
\hline-1.00 & 4.16 & 0.58 & 0.36 & & 0.32 & 0.141 & 0.43 & -0.44 \\
\hline-0.70 & 4.38 & 0.59 & 0.37 & -0.46 & 0.35 & 0.156 & 0.48 & -0.45 \\
\hline-0.50 & 4.55 & 0.59 & 0.37 & & 0.40 & 0.169 & .49 & -0.45 \\
\hline-0.20 & 4.86 & 0.60 & 0.37 & -0.46 & 0.50 & 0.183 & 0.53 & -0.46 \\
\hline 0.00 & 5.04 & 0.60 & 0.38 & -0.48 & 0.57 & 0.193 & 0.55 & -0.46 \\
\hline 0.20 & 5.22 & 0.58 & 0.37 & -0.46 & 0.65 & 0.188 & 0.54 & -0.45 \\
\hline 0.50 & 5.49 & 0.58 & 0.37 & -0.48 & 0.73 & 0.184 & 0.56 & -0.46 \\
\hline 0.70 & 5.64 & 0.57 & 0.37 & -0.48 & 0.77 & 0.176 & 0.56 & -0.45 \\
\hline 1.00 & 5.97 & 0.55 & 0.36 & -0.47 & 0.81 & 0.159 & 0.54 & -0.44 \\
\hline 1.50 & 6.46 & 0.53 & 0.35 & -0.49 & 0.94 & 0.122 & 0.50 & -0.41 \\
\hline 2.00 & 6.99 & 0.50 & 0.33 & -0.48 & 1.05 & 0.079 & 0.42 & -0.36 \\
\hline 2.50 & 7.51 & 0.45 & 0.31 & -0.49 & 1.10 & 0.043 & 0.27 & -0.26 \\
\hline 3.00 & 7.96 & 0.40 & 0.28 & -0.49 & 1.13 & 0.029 & 0.16 & -0.17 \\
\hline
\end{tabular}

Table A.5: Measurements at $x_{1} / M=90$ 


\begin{tabular}{|c|c|c|c|c|c|c|c|c|}
\hline$\frac{x_{2}}{M}$ & $\begin{array}{c}\overline{U_{1}} \\
(\mathrm{~m} / \mathrm{s})\end{array}$ & $\begin{array}{c}u_{1}^{\prime} \\
(\mathrm{m} / \mathrm{s}) \\
\end{array}$ & $\begin{array}{c}u_{\cdot 2} \\
(\mathrm{~m} / \mathrm{s}) \\
\end{array}$ & $\rho_{u_{1} u_{2}}$ & $\begin{array}{c}\bar{T} \\
\left({ }^{\circ} \mathrm{I}\right) \\
\end{array}$ & $\begin{array}{c}\theta^{\prime} \\
\left({ }^{\circ} I I^{\prime}\right) \\
\end{array}$ & $\rho_{u_{2} O}$ & $\rho_{\mathrm{H}_{2} \mathrm{O}}$ \\
\hline-2.50 & 3.17 & 0.56 & 0.43 & -0.42 & 0.10 & 0.081 & 0.13 & -0.35 \\
\hline-2.00 & 3.53 & 0.60 & 0.37 & -0.42 & 0.17 & 0.102 & 0.28 & -0.39 \\
\hline-1.50 & 3.87 & 0.63 & 0.39 & -0.44 & 0.22 & 0.124 & 0.37 & -0.42 \\
\hline-1.00 & 4.28 & 0.65 & 0.41 & -0.46 & 0.34 & 0.147 & 0.45 & -0.46 \\
\hline-0.70 & 4.47 & 0.65 & 0.41 & -0.45 & 0.40 & 0.157 & 0.47 & -0.45 \\
\hline-0.50 & 4.64 & 0.65 & 0.42 & -0.47 & 0.46 & 0.166 & 0.50 & -0.46 \\
\hline-0.30 & 4.87 & 0.65 & 0.42 & -0.47 & 0.51 & 0.171 & 0.51 & -0.46 \\
\hline 0.00 & 5.14 & 0.67 & 0.42 & -0.49 & 0.56 & 0.183 & 0.54 & -0.47 \\
\hline 0.30 & 5.43 & 0.67 & 0.42 & -0.48 & 0.66 & 0.182 & 0.55 & -0.47 \\
\hline 0.50 & 5.62 & 0.66 & 0.42 & -0.49 & 0.72 & 0.178 & 0.55 & -0.46 \\
\hline 0.70 & 5.81 & 0.66 & 0.42 & -0.49 & 0.77 & 0.177 & 0.55 & -0.46 \\
\hline 1.00 & 6.07 & 0.64 & 0.41 & -0.50 & 0.80 & 0.158 & 0.55 & -0.46 \\
\hline 1.50 & 6.58 & 0.60 & 0.40 & -0.51 & 0.91 & 0.130 & 0.52 & -0.43 \\
\hline 2.00 & 7.10 & 0.56 & 0.38 & -0.51 & 0.99 & 0.092 & 0.49 & -0.39 \\
\hline 2.50 & 7.62 & 0.50 & 0.35 & -0.51 & 1.08 & 0.057 & 0.40 & -0.35 \\
\hline
\end{tabular}

Table A.6: Measurements at $x_{1} / M=105$ 


\begin{tabular}{|c|c|}
\hline 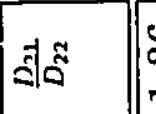 & 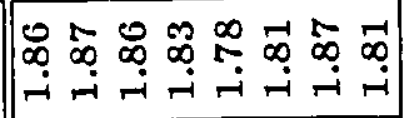 \\
\hline 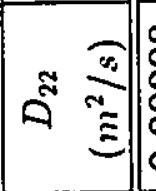 & 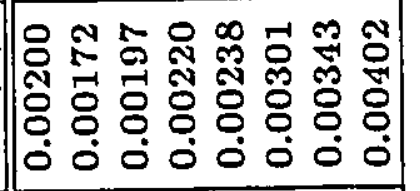 \\
\hline$=0$ & 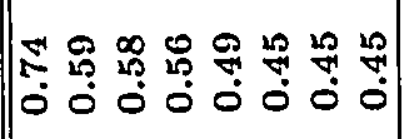 \\
\hline$\triangleq 1$ & 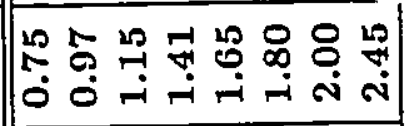 \\
\hline 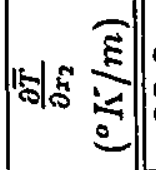 & 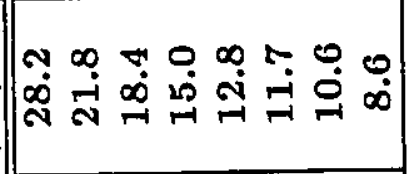 \\
\hline$=\overparen{E}$ & 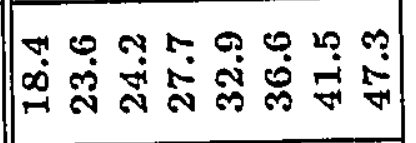 \\
\hline$\stackrel{\Xi}{\Xi}$ & 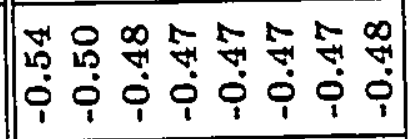 \\
\hline$\stackrel{\Xi}{\Xi}$ & 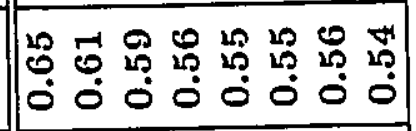 \\
\hline - 8 & 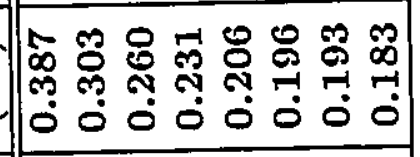 \\
\hline$\because \frac{2}{\xi}$ & 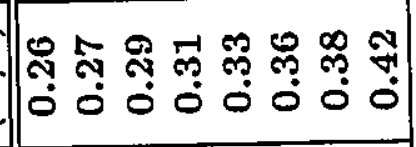 \\
\hline$=\frac{\widehat{D}}{\mathrm{E}}$ & 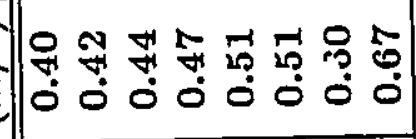 \\
\hline $15-\frac{2}{E}$ & 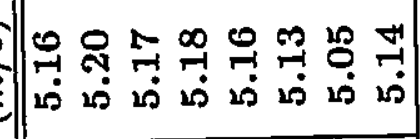 \\
\hline मीट & : \\
\hline
\end{tabular}

